\title{
Angular Momentum Diagrams
}

\author{
Paul E.S. Wormer ${ }^{1}$ and Josef Paldus ${ }^{2}$ \\ ${ }^{1}$ Theoretical Chemistry, Institute for Molecules and Materials, Radboud University Nijmegen, \\ Toernooiveld, 6525 ED Nijmegen, The Netherlands \\ E-mail: pwormer@theochem.ru.nl \\ ${ }^{2}$ Department of Applied Mathematics, Department of Chemistry, and Guelph-Waterloo Center for Graduate \\ Work in Chemistry-Waterloo Campus, University of Waterloo, Waterloo, Ontario, Canada, N2L $3 G 1$ \\ E-mail:paldus@scienide.uwaterloo.ca
}

\begin{abstract}
Starting from the simplest possible building blocks - a ket, a bra, a time-reversed ket, and a time-reversed braa diagrammatic formalism is developed for angular momentum coupling problems. The formalism comprises Clebsch-Gordan coefficients as well as $3 \mathrm{jm}$-symbols. The idea of constructing invariants (internal lines) by contracting contragredient pairs of quantities is emphasized throughout. The Clebsch-Gordan series, and its extension to the coupling of more than two angular momenta, is introduced algebraically and diagrammatically. Recoupling between bases obtained in different coupling schemes is introduced and the connection between recoupling coefficients and irreducible $3 n j$-symbols is derived diagrammatically. The well-known diagrammatic rules due to Jucys and co-workers are derived by group theoretical means and simple rules for their practical exploitation are presented.
\end{abstract}

\section{Contents}

1. Introduction

2. The essentials of SU(2)

2.2. Second-quantized representation

2.3. Contragredient representations 9

2.4. Time reversal $r$

2.5. Clebsch-Gordan series $\quad 13$

3. Diagrams $\quad 16$

3.1. Basic diagrammatic building blocks 16

3.2. Clebsch-Gordan diagrams 19

3.3. $1 \mathrm{jm}$-symbol 22

3.4. Generalized Clebsch-Gordan coefficients 23

3.5. $3 j m$-symbols $r$

3.6. Transition from CG coefficients to $3 j m$-symbols 29

4. Basic rules for angular momentum diagrams $\quad 31$

4.1. Removal of a zero line $\quad 32$

4.2. Factorization rules $\quad 34$

$\begin{array}{lr}\text { 4.3. Separation rules } & 39\end{array}$

5. Irreducible closed diagrams $\quad 43$

5.1. $1 j$ - and $3 j$-symbols $\quad 44$

5.2. Recoupling and the $6 j$ - and $9 j$-symbols $\quad 44$

5.3. Properties of the $6 j$-symbol 48

The $6 j$-symbol is invariant under any permutation of its vertices 48

The $6 j$-symbol is invariant with respect to the interchange of the rows in any two of its columns 49

ADVANCES IN QUANTUM CHEMISTRY, VOLUME 51, pp. 59-124, (2006)

ISSN: 0065-3276 DOI: 10.1016/S0065-3276(06)51002-0

All rights reserved 
5.4. Properties of the $9 j$-symbol $\quad 52$

5.5. Higher $3 n j$-symbols $\quad 55$

6. Concluding remarks $\quad 55$

$\begin{array}{lr}\text { Acknowledgement } & 57\end{array}$

Appendix: Summary of the graphical rules $\quad 57$

$\begin{array}{ll}\text { Basic units } & 57\end{array}$

Basic rules $\quad 57$

jm-symbol $r$

$\begin{array}{ll}\text { Clebsch-Gordan coefficients and diagrams } & 58\end{array}$

$j m$-symbols and diagrams $r$

Transition from CG to 3jm-diagrams $\quad 60$

Diagram manipulation $\quad 60$

Basic irreducible diagrams; $3 n j$-symbols $\quad 62$

References

\section{INTRODUCTION}

The origins of the coupling problem for angular momenta can be traced back to the earlypurely mathematical—work on invariant theory (Refs. [1,2]) by (Rudolf Friedrich) Alfred Clebsch (1833-1872) and Paul (Albert) Gordan (1837-1912), see Section 2.5. Even before the birth of quantum mechanics the formal analogy between chemical valence theory and binary invariant theory was recognized by eminent mathematicians as Sylvester [3], Clifford [4], and Gordan and Alexejeff [5]. The analogy, lacking a physical basis at the time, was criticized heavily by the mathematician E. Study and ignored completely by the chemistry community of the 1890s. After the advent of quantum mechanics it became clear, however, that chemical valences arise from electron-spin couplings, see, e.g., [6] and that electron-spin functions are, in fact, binary forms of the type studied by Gordan and Clebsch.

Angular momentum is of great physical importance because it represents a constant of the motion for rotationally invariant systems. Although this observation is as true in classical mechanics as it is in quantum mechanics, it is not of great help in solving the problems of classical mechanics. However, quantum mechanics and the aid of symmetry in solving the Schrödinger equation, brought angular momentum theory to its prominence. From a mathematical viewpoint, this theory is equivalent to the study of the representation theory of the unitary unimodular group SU(2). There is hardly a textbook on quantum theory that would not include a chapter or a section dealing with the orbital and spin angular momenta, not to mention a number of specialized publications on the subject, see, e.g., Refs. [7-17]. (For a more exhaustive reference list Ref. [13] may be consulted.)

The present review introduces the diagrammatic representation of angular momentum theory. This representation is mainly due to Jucys and his co-workers. In their 1962 book [18] Jucys, Levinson, and Vanagas (JLV) based their diagrams completely on $3 j$-symbols (which for reasons that will become clear later, will be referred to as $3 j m$-symbols from here on). In a later book Jucys and Bandzaitis [19] worked solely with Clebsch-Gordan (CG) coefficients. These coefficients have the advantage that they allow the formulation of an angular momentum problem in the most natural way, namely in terms of eigenkets and eigenbras of angular momentum operators. However, CG coefficients have

Due to transcriptions from Lithuanian (which uses the Latin alphabet) into the Cyrillic alphabet and back, one often finds different spellings, e.g., Yutsis, Yuzis, Ioucis, etc. The correct Lithuanian spelling is Jucys. 
the disadvantage that their symmetry relations are awkward and that the reduction of an angular momentum problem to irreducible quantities (i.e., $6 j-, 9 j-, \ldots, 3 n j$-symbols) is difficult. El Baz and Castel [20] introduced a graphical formalism that incorporates both types of coefficients: $3 \mathrm{jm}$-symbols and CG coefficients. The formalism introduced below is inspired by their work, but avoids two problems present in their formalism. In the first place El Baz and Castel identify a bra with a time-reversed ket and although both quantities transform identically under SU(2), they are not the same. Second, they do not distinguish diagrammatically the unique pair of quantum numbers in a $C G$ coefficient, which is an important characteristic of a CG coefficient.

In their 1993 book [21] Brink and Satchler (BS) present a diagrammatic formalism that has a large overlap with the JLV $3 \mathrm{jm}$-formalism. The two differ mainly in that BS suppress line orientations when these may be freely flipped (i.e., without introduction of phase). Furthermore, BS claim that their approach has the advantage over the JLV approach that CG coefficients can be handled, which allegedly cannot be done in the JLV formalism. Since BS simply use the fact that a CG coefficient is proportional to a $3 \mathrm{jm}$-symbol and since they do not attempt to represent the proportionality factor diagrammatically, it is hard to endorse this advantage.

Before introducing diagrams, we shall briefly review the necessary parts of angular momentum theory in the first few sections. The components of the angular momentum operator span the Lie algebra of $\mathrm{SO}(3)$ (the group of unimodular (unit determinant) orthogonal (rotation) operators on real three-dimensional vector space), as well as of SU(2) (which consists of unimodular, unitary operators on complex two-dimensional vector space), and so the quantum theory of angular momentum is closely intertwined with the representation theory of these two groups.

The group SU(2) has two simplifying properties that related groups, such as SU(3), do not possess. In the first place, the conjugacy classes of $S U(2)$ are ambivalent, i.e., contain with an element $U \in \mathrm{SU}(2)$ also its inverse $U^{-1}$. This implies that the characters of $U$ and $U^{-1}$ are equal in any representation (rep). Since in a unitary rep the character of an element and of its inverse are each others complex conjugate, it follows from the ambivalent nature of its classes that all characters of any unitary rep of SU(2) are real. As a result, every rep is equivalent to its complex conjugate rep. The second simplifying property of $\mathrm{SU}(2)$ is that its irreducible representations (irreps) are multiplicity free. This means that the reduction of a direct product of two irreps will yield any irrep at most once. Wigner [22] calls groups that possess these two properties simply reducible. If SU(2) and $\mathrm{SO}(3)$ had not been simply reducible, a diagrammatic description of their representation theory would have been much more cumbersome.

The following notes also introduce the concept of mixed $3 j m$-symbols, recoupling, and the $6 j-, 9 j-, \ldots, 3 n j$-symbols. It is the belief of the authors that $3 n j$-coefficients are easier to grasp diagrammatically than algebraically.

\section{THE ESSENTIALS OF SU(2)}

We review the necessary theory of the group SU(2). First, we show that a general element can be written in exponential form and then we introduce a second-quantized basis carrying irreps of SU(2). The concept of standard and contrastandard representations is introduced and the connection with time reversal is pointed out. Finally, the Clebsch-Gordan series is discussed. 


\subsection{Exponential form of $\mathrm{SU}(2)$ elements}

The Pauli spin matrices [23]

$$
\sigma_{x}=\left(\begin{array}{cc}
0 & 1 \\
1 & 0
\end{array}\right), \quad \sigma_{y}=\left(\begin{array}{cc}
0 & -\mathrm{i} \\
\mathrm{i} & 0
\end{array}\right), \quad \boldsymbol{\sigma}_{z}=\left(\begin{array}{cc}
1 & 0 \\
0 & -1
\end{array}\right)
$$

satisfy the relations

$$
\boldsymbol{\sigma}_{j} \boldsymbol{\sigma}_{k}=\delta_{j k} \mathbf{1}+\mathrm{i} \varepsilon_{j k l} \boldsymbol{\sigma}_{l},
$$

where $\varepsilon_{j k l}$ (the Levi-Civita tensor) is the totally antisymmetric tensor of rank 3 defined by

$$
\varepsilon_{i j k}=\operatorname{det}\left(\hat{e}_{i}, \hat{e}_{j}, \hat{e}_{k}\right) \quad \text { for } i, j, k=1,2,3,
$$

and $\hat{e}_{1}, \hat{e}_{2}, \hat{e}_{3}$ form a right-handed orthonormal set of vectors. From equation (2) follows immediately that

$$
\boldsymbol{\sigma}_{j} \boldsymbol{\sigma}_{k}-\boldsymbol{\sigma}_{k} \boldsymbol{\sigma}_{j} \equiv\left[\boldsymbol{\sigma}_{j}, \boldsymbol{\sigma}_{k}\right]=2 \mathrm{i} \varepsilon_{j k l} \boldsymbol{\sigma}_{l} .
$$

Thus, defining operators $\hat{s}_{j}$ by the action on the usual spin- $\frac{1}{2}$ functions

$$
\hat{s}_{j}(|\alpha\rangle,|\beta\rangle)=(|\alpha\rangle,|\beta\rangle) \frac{1}{2} \sigma_{j}, \quad j=1,2,3,
$$

we obtain the usual angular momentum commutation relations

$$
\left[\hat{s}_{j}, \hat{s}_{k}\right]=\mathrm{i} \varepsilon_{j k l} \hat{s}_{l} .
$$

It is easily seen that a $2 \times 2$ unimodular $(\mathrm{det}=1)$, unitary matrix is parametrized by two complex numbers, because

$$
\mathbf{U}^{-1} \equiv\left(\begin{array}{ll}
u_{11} & u_{12} \\
u_{21} & u_{22}
\end{array}\right)^{-1}=\operatorname{det}(\mathbf{U})^{-1}\left(\begin{array}{cc}
u_{22} & -u_{12} \\
-u_{21} & u_{11}
\end{array}\right)=\mathbf{U}^{\dagger}=\left(\begin{array}{ll}
u_{11}^{*} & u_{21}^{*} \\
u_{12}^{*} & u_{22}^{*}
\end{array}\right) .
$$

Since $\operatorname{det}(\mathbf{U})=1$, it follows, if we set $u_{11} \equiv u$ and $u_{12} \equiv v$, that

$$
\mathbf{U}=\left(\begin{array}{cc}
u & v \\
-v^{*} & u^{*}
\end{array}\right), \quad u, v \in \mathbb{C} .
$$

We can introduce real parameters $a_{i}$,

$$
\left(\begin{array}{cc}
u & v \\
-v^{*} & u^{*}
\end{array}\right)=\left(\begin{array}{cc}
a_{0}-\mathrm{i} a_{3} & -\mathrm{i} a_{1}-a_{2} \\
-\mathrm{i} a_{1}+a_{2} & a_{0}+\mathrm{i} a_{3}
\end{array}\right)=a_{0} \mathbf{1}-\mathrm{i} \overrightarrow{\boldsymbol{\sigma}} \cdot \vec{a}
$$

with $\vec{a} \equiv\left(a_{1}, a_{2}, a_{3}\right)$ and where

$$
\operatorname{det}(\mathbf{U})=|u|^{2}+|v|^{2}=a_{0}^{2}+a_{1}^{2}+a_{2}^{2}+a_{3}^{2}=1 .
$$

This parametrization is often referred to as the Euler-Rodrigues parametrization. It indicates that as an analytic manifold the Lie group SU(2) is the three-dimensional surface of the unit sphere $S^{3}$ in $\mathbb{R}^{4}$. The sphere $S^{3}$ is covered exactly once (except for the poles $\varphi=0$ and $\varphi=2 \pi)$ by $\left(a_{0}, \vec{a}\right)=\left(\cos \frac{\varphi}{2}, \vec{n} \sin \frac{\varphi}{2}\right)$, where $\vec{n}$ is a vector that ranges over the whole unit sphere $S^{2}$ in $\mathbb{R}^{3}$ and $0 \leqslant \varphi \leqslant 2 \pi$. Hence,

$$
\mathbf{U}(\vec{n}, \varphi)=\mathbf{1} \cos \frac{\varphi}{2}-\mathrm{i}(\overrightarrow{\boldsymbol{\sigma}} \cdot \vec{n}) \sin \frac{\varphi}{2} .
$$


Since $\vec{n}$ is a unit vector and $\vec{n} \times \vec{n}=\overrightarrow{0}$, we have, using summation convention, from equation (2):

$$
(\overrightarrow{\boldsymbol{\sigma}} \cdot \vec{n})^{2} \equiv n_{j} n_{k} \boldsymbol{\sigma}_{j} \boldsymbol{\sigma}_{k}=n_{j} n_{k} \delta_{j k} \mathbf{1}+\mathrm{i}(\vec{n} \times \vec{n})_{l} \boldsymbol{\sigma}_{l}=\mathbf{1} .
$$

This implies for positive integer $p$

$$
(\overrightarrow{\boldsymbol{\sigma}} \cdot \vec{n})^{2 p}=\mathbf{1} \text { and }(\overrightarrow{\boldsymbol{\sigma}} \cdot \vec{n})^{2 p+1}=\overrightarrow{\boldsymbol{\sigma}} \cdot \vec{n},
$$

so that the general element of SU(2) can be written as

$$
\mathbf{U}(\vec{n}, \varphi)=\mathrm{e}^{-\mathrm{i}(\vec{\sigma} \cdot \vec{n}) \varphi / 2} .
$$

We note, finally, that a rotation by $2 \pi$ over a fixed axis $\vec{n}$ does not correspond to the identity operator, but

$$
\mathbf{U}(\vec{n}, 2 \pi)=\mathbf{1} \cos \pi-\mathrm{i}(\overrightarrow{\boldsymbol{\sigma}} \cdot \vec{n}) \sin \pi=-\mathbf{1} \quad(\neq \mathbf{U}(\vec{n}, 0)=\mathbf{1}) .
$$

We thus have to consider $0 \leqslant \varphi \leqslant 4 \pi$ to cover the whole of SU(2) (and must restrict the range of $\vec{n}$ to a unit hemisphere if the group is to be covered once).

\subsection{Second-quantized representation}

Schwinger [24] introduced a second-quantized representation of angular momentum operators $\mathcal{J}_{x}, \mathcal{J}_{y}, \mathcal{J}_{z}$, that satisfy the usual commutation relations

$$
\left[\mathcal{J}_{j}, \mathcal{J}_{k}\right]=\mathrm{i} \varepsilon_{j k l} \mathcal{J}_{l}, \quad j, k, l=x, y, z .
$$

He represented also their eigenstates, which span carrier spaces of the SU(2) irreps, in second-quantized form. These eigenstates of the total angular momentum operators $\mathcal{J}^{2}$ and $\mathcal{J}_{z}$, designated by $|j, m\rangle$, are labeled by the quantum numbers $j$ and $m$, with $j$ labeling a given irrep and $m$ the basis states of this irrep, $m=-j,-j+1, \ldots, j-1, j$, and transform in the usual manner under the action of the step up and step down operators $\mathcal{J}_{+} \equiv \mathcal{J}_{x}+\mathrm{i} \mathcal{J}_{y}$ and $\mathcal{J}_{-} \equiv \mathcal{J}_{x}-\mathrm{i} \mathcal{J}_{y}$, respectively, namely

$$
\begin{aligned}
& \mathcal{J}^{2}|j, m\rangle=|j, m\rangle j(j+1), \\
& \mathcal{J}_{z}|j, m\rangle=|j, m\rangle m, \\
& \mathcal{J}_{ \pm}|j, m\rangle=|j, m \pm 1\rangle \sqrt{(j \mp m)(j \pm m+1)} .
\end{aligned}
$$

Since Schwinger's second-quantization representation is very compact and facilitates the transition to the corresponding contragredient form, it is essential for the diagrammatic representation considered here.

In order to introduce Schwinger's formalism it is useful to recall the well-known secondquantized representation of a one-dimensional harmonic oscillator with the Hamiltonian

$$
H=\hbar \omega\left(b^{\dagger} b+\frac{1}{2}\right)
$$

where the creation and annihilation operators $b^{\dagger}$ and $b$ satisfy the boson commutation relation

$$
\left[b, b^{\dagger}\right]=1, \quad\left[b^{\dagger}, b^{\dagger}\right]=[b, b]=0,
$$


and the energies $E_{n}$ and eigenstates $|n\rangle$ are given by

$$
E_{n}=\hbar \omega\left(n+\frac{1}{2}\right) \quad \text { and } \quad|n\rangle=\frac{\left(b^{\dagger}\right)^{n}}{\sqrt{n !}}|0\rangle
$$

with the action

$$
\begin{aligned}
& b|0\rangle=0, \\
& b|n\rangle=|n-1\rangle \sqrt{n}, \\
& b^{\dagger}|n\rangle=|n+1\rangle \sqrt{n+1} .
\end{aligned}
$$

The normalization of $|n\rangle$ is easily checked by application of the following "differentiation" rule

$$
\left[b,\left(b^{\dagger}\right)^{k}\right]=k\left(b^{\dagger}\right)^{k-1} .
$$

This result can easily be proved by mathematical induction. Considering the matrix element $(k \geqslant n)$

$$
\langle k \mid n\rangle=\frac{1}{\sqrt{k ! n !}}\left\langle 0\left|b^{k}\left(b^{\dagger}\right)^{n}\right| 0\right\rangle=\frac{1}{\sqrt{k ! n !}} n !\left\langle 0\left|b^{k-n}\right| 0\right\rangle=\delta_{k, n},
$$

we find that the kets $|n\rangle(n=0,1, \ldots)$ are orthonormal.

Returning to $\mathrm{SU}(2)$, we recall that this is the dynamic symmetry group of the isotropic two-dimensional harmonic oscillator. The time-independent Schrödinger equation of such an oscillator separates into two uncoupled one-dimensional equations. The Hamiltonian is the sum of two single-mode Hamiltonians given in equation (10),

$$
H=\hbar \omega\left(\sum_{i=1}^{2} b_{i}^{\dagger} b_{i}+1\right)
$$

Its eigenstates are simply tensorial products,

$$
\left|n_{1}, n_{2}\right\rangle:=\left|n_{1}\right\rangle \otimes\left|n_{2}\right\rangle .
$$

Since $b_{1}$ and $b_{2}$ are related to independent (uncoupled) vibrational modes, the following commutation relations hold,

$$
\left[b_{i}, b_{j}^{\dagger}\right]=\delta_{i j}, \quad\left[b_{i}, b_{j}\right]=\left[b_{i}^{\dagger}, b_{j}^{\dagger}\right]=0, \quad i, j=1,2,
$$

and their action on kets is the same as before, $c f$. equations (13a)-(13c). We can relate the eigenstates $|j, m\rangle$ to $\left|n_{1}, n_{2}\right\rangle$ by

$$
n_{1}=j+m \quad \text { and } \quad n_{2}=j-m .
$$

Thus,

$$
\begin{aligned}
|j, m\rangle & \equiv\left|n_{1}, n_{2}\right\rangle=\frac{\left(b_{1}^{\dagger}\right)^{n_{1}}\left(b_{2}^{\dagger}\right)^{n_{2}}}{\sqrt{n_{1} ! n_{2} !}}|0\rangle \\
& =\frac{\left(b_{1}^{\dagger}\right)^{j+m}\left(b_{2}^{\dagger}\right)^{j-m}}{\sqrt{(j+m) !(j-m) !}}|0\rangle,
\end{aligned}
$$


where $|0\rangle \equiv|j=0, m=0\rangle=\left|n_{1}=0, n_{2}=0\right\rangle$ and $\mathcal{J}_{i}|0\rangle=0(i=x, y, z)$. Equations $(9 \mathrm{c})$ can be rewritten as

$$
\begin{aligned}
& \mathcal{J}_{+}\left|n_{1}, n_{2}\right\rangle=\left|n_{1}+1, n_{2}-1\right\rangle \sqrt{\left(n_{1}+1\right) n_{2}}, \\
& \mathcal{J}_{-}\left|n_{1}, n_{2}\right\rangle=\left|n_{1}-1, n_{2}+1\right\rangle \sqrt{n_{1}\left(n_{2}+1\right)},
\end{aligned}
$$

so that

$$
\begin{aligned}
& \mathcal{J}_{+}=b_{1}^{\dagger} b_{2} \equiv \mathcal{E}_{12}, \\
& \mathcal{J}_{-}=b_{2}^{\dagger} b_{1} \equiv \mathcal{E}_{21} .
\end{aligned}
$$

The $j=0$ "vacuum" state $|0\rangle$ is rotationally invariant, so that

$$
\hat{U}|0\rangle=|0\rangle, \quad \hat{U} \in \mathrm{SU}(2) .
$$

Writing for the general action of $\hat{U}$ on basis vectors $|\sigma\rangle \equiv b_{\sigma}^{\dagger}|0\rangle$, which span a given $\mathrm{SU}(2)$ irrep,

$$
\hat{U}|\sigma\rangle \equiv|\tilde{\sigma}\rangle=\sum_{\mu}|\mu\rangle U_{\mu \sigma},
$$

or, equivalently,

$$
\tilde{b}_{\sigma}^{\dagger}=\sum_{\mu} b_{\mu}^{\dagger} U_{\mu \sigma}, \quad \tilde{b}_{\sigma}^{\dagger}|0\rangle=|\tilde{\sigma}\rangle,
$$

we see that the rotational invariance of $|0\rangle$ and the unitarity of $\hat{U}$ imply

$$
\hat{U} b_{\sigma}^{\dagger}|0\rangle=\hat{U} b_{\sigma}^{\dagger} \hat{U}^{\dagger} \hat{U}|0\rangle=\hat{U} b_{\sigma}^{\dagger} \hat{U}^{\dagger}|0\rangle=\tilde{b}_{\sigma}^{\dagger}|0\rangle=\sum_{\mu} b_{\mu}^{\dagger} U_{\mu \sigma}|0\rangle,
$$

or, succinctly,

$$
\hat{U} b_{\sigma}^{\dagger} \hat{U}^{\dagger}=\sum_{\mu} b_{\mu}^{\dagger} U_{\mu \sigma}, \quad \hat{U} \in \mathrm{SU}(2) .
$$

We see that the total Hamiltonian in equation (15), which contains implicitly a sum of kinetic and potential energy terms, is invariant under $\mathrm{SU}(2)$, while the separate kinetic and potential energy terms are invariant under $\mathrm{SO}(2) \subset \mathrm{SU}(2)$.

Generally we can write

$$
\mathcal{J}_{k}=\frac{1}{2}\left(\begin{array}{ll}
b_{1}^{\dagger} & b_{2}^{\dagger}
\end{array}\right) \sigma_{k}\left(\begin{array}{l}
b_{1} \\
b_{2}
\end{array}\right), \quad \text { with } k=x, y, z .
$$

Equivalently, using the operators $\mathcal{E}_{i j}$ introduced above, $i, j=1,2$,

$$
\mathcal{E}_{i j}=b_{i}^{\dagger} b_{j}
$$

we find that

$$
\begin{aligned}
\mathcal{J}_{x} & =\frac{1}{2}\left(\mathcal{E}_{12}+\mathcal{E}_{21}\right), \\
\mathcal{J}_{y} & =-\frac{\mathrm{i}}{2}\left(\mathcal{E}_{12}-\mathcal{E}_{21}\right), \\
\mathcal{J}_{z} & =\frac{1}{2}\left(\mathcal{E}_{11}-\mathcal{E}_{22}\right),
\end{aligned}
$$


and

$$
\begin{aligned}
\hat{\mathcal{J}}^{2} & :=\hat{\mathcal{J}}_{x}^{2}+\hat{\mathcal{J}}_{y}^{2}+\hat{\mathcal{J}}_{z}^{2} \\
& =\frac{1}{2}\left(\hat{\mathcal{J}}_{+} \hat{\mathcal{J}}_{-}+\hat{\mathcal{J}}_{-} \hat{\mathcal{J}}_{+}\right)+\hat{\mathcal{J}}_{z}^{2} \\
& =\frac{1}{2}\left(\mathcal{E}_{12} \mathcal{E}_{21}+\mathcal{E}_{21} \mathcal{E}_{12}\right)+\frac{1}{4}\left(\mathcal{E}_{11}-\mathcal{E}_{22}\right)^{2} .
\end{aligned}
$$

It is important to note that the operators $\mathcal{E}_{i j}$ possess the commutation relations

$$
\left[\mathcal{E}_{i j}, \mathcal{E}_{m n}\right]=\delta_{j m} \mathcal{E}_{i n}-\delta_{i n} \mathcal{E}_{m j}, \quad i, j, m, n=1,2,
$$

characterizing the generators of the general linear group GL(2) and thus of its subgroup $\mathrm{SU}(2)$.

The action of these operators on an arbitrary state $|j, m\rangle$ is now easily checked to be

$$
\begin{aligned}
\mathcal{E}_{11}|j m\rangle & =b_{1}^{\dagger} b_{1}|j m\rangle \\
& =[(j+m) !(j-m) !]^{-1 / 2}(j+m) b_{1}^{\dagger}\left(b_{1}^{\dagger}\right)^{j+m-1}\left(b_{2}^{\dagger}\right)^{j-m}|0\rangle \\
& =(j+m)|j m\rangle,
\end{aligned}
$$

where we used the differentiation rule:

$$
b_{1}\left(b_{1}^{\dagger}\right)^{j+m}\left(b_{2}^{\dagger}\right)^{j-m}|0\rangle=(j+m)\left(b_{1}^{\dagger}\right)^{j+m-1}\left(b_{2}^{\dagger}\right)^{j-m}|0\rangle .
$$

In the same way,

$$
\begin{aligned}
& \mathcal{E}_{22}|j m\rangle=(j-m)|j m\rangle, \\
& \mathcal{E}_{12}|j m\rangle=[(j-m)(j+m+1)]^{1 / 2}|j, m+1\rangle, \\
& \mathcal{E}_{21}|j m\rangle=[(j+m)(j-m+1)]^{1 / 2}|j, m-1\rangle .
\end{aligned}
$$

Using these relationships, we easily recover equations $(9 a)-(9 c)$, verifying that the Schwinger states (18) possess the usual properties of angular momentum states and span a single $(2 j+1)$-dimensional irrep of SU(2) labeled by $j$.

Note that for $j=\frac{1}{2}, m=\frac{1}{2}$, we recover the standard spin up and spin down functions,

$$
\begin{aligned}
& \left|\frac{1}{2}, \frac{1}{2}\right\rangle=b_{1}^{\dagger}|0\rangle \equiv \alpha(1), \\
& \left|\frac{1}{2},-\frac{1}{2}\right\rangle=b_{2}^{\dagger}|0\rangle \equiv \beta(1),
\end{aligned}
$$

spanning the defining representation of SU(2). Due to the bosonic character of the $b_{i}^{\dagger}$, the states (18) are symmetric under permutation of the creation operators. This implies that the kets (18) are the second-quantized representation of a system consisting of $2 j$ spin- $\frac{1}{2}$ particles in a state of highest total spin $j$. Thus, for example,

$$
\begin{aligned}
& \frac{1}{\sqrt{2}} b_{1}^{\dagger} b_{1}^{\dagger}|0\rangle \leftrightarrow \alpha(1) \alpha(2), \\
& b_{1}^{\dagger} b_{2}^{\dagger}|0\rangle \leftrightarrow \frac{\alpha(1) \beta(2)+\beta(1) \alpha(2)}{\sqrt{2}}, \\
& \frac{1}{\sqrt{2}} b_{2}^{\dagger} b_{2}^{\dagger}|0\rangle \leftrightarrow \beta(1) \beta(2) .
\end{aligned}
$$


To express the antisymmetric two-particle singlet state

$$
\frac{\alpha(1) \beta(2)-\beta(1) \alpha(2)}{\sqrt{2}}
$$

in second-quantized form, the boson operators must be equipped with a second subscript [25].

At this point it would be easy to construct the actual matrix irrep $\mathbf{D}^{(j)}$ as a polynomial in the matrix elements $U_{\mu \sigma}(c f$. equation (25)) by using

$$
\hat{U} b_{1}^{\dagger} b_{1}^{\dagger} b_{1}^{\dagger} \cdots|0\rangle=\left(\hat{U} b_{1}^{\dagger} \hat{U}^{\dagger}\right)\left(\hat{U} b_{1}^{\dagger} \hat{U}^{\dagger}\right)\left(\hat{U} b_{1}^{\dagger} \hat{U}^{\dagger}\right) \cdots \hat{U}|0\rangle
$$

and $\hat{U}|0\rangle=|0\rangle$. Since we do not need the explicit form of $\mathbf{D}^{(j)}$ we skip this construction.

\subsection{Contragredient representations}

Recall that a non-singular matrix $\mathbf{A}$ is in one-to-one correspondence with the contragredient matrix $\overline{\mathbf{A}}:=\left(\mathbf{A}^{-1}\right)^{\mathrm{T}}$. For unitary matrices we have $\overline{\mathbf{U}}=\left(\mathbf{U}^{\dagger}\right)^{\mathrm{T}}=\mathbf{U}^{*}$, i.e., a unitary matrix and its contragredient counterpart are connected by complex conjugation. In the terminology of Fano and Racah [9] the unitary contrastandard matrix rep $\overline{\mathbf{D}}(g)$ of a group $G$ is the complex conjugate $\mathbf{D}(g)^{*}$ of the unitary standard matrix rep $\mathbf{D}(g)$. The concept of contragredience is important because it leads by contraction to group invariants. Indeed, suppose $\chi_{i}$ and $\bar{\chi}_{i}$ are bases of $n$-dimensional vector spaces $V$ and $\bar{V}$ that carry $\mathbf{D}(g)$ and $\overline{\mathbf{D}}(g)$, respectively, of the same group $G$. That is, for all $g \in G$,

$$
g\left(\chi_{i}\right)=\sum_{j=1}^{n} \chi_{j} D(g)_{j i} \quad \text { and } \quad g\left(\bar{\chi}_{i}\right)=\sum_{j=1}^{n} \bar{\chi}_{j} \bar{D}(g)_{j i} .
$$

The contraction of $\chi_{i}$ and $\bar{\chi}_{i}$ satisfies

$$
\begin{aligned}
\sum_{i=1}^{n} g\left(\chi_{i}\right) g\left(\bar{\chi}_{i}\right) & =\sum_{i, j, k} \chi_{j} \bar{\chi}_{k} D(g)_{j i} \bar{D}(g)_{k i} \\
& =\sum_{j, k} \chi_{j} \bar{\chi}_{k} \delta_{j k} \\
& =\sum_{i=1}^{n} \chi_{i} \bar{\chi}_{i}
\end{aligned}
$$

and is therefore a group invariant.

For general groups the standard rep $\mathbf{D}(g)$ and the contrastandard rep $\overline{\mathbf{D}}(g)$ are not necessarily equivalent. It was pointed out in the Introduction that for groups with only ambivalent conjugacy classes the standard and contrastandard matrix rep are equivalent, because their characters are real, and so, since the classes of SU(2) are ambivalent, its standard and contrastandard reps are equivalent.

Consider an orthonormal basis (for instance a Schwinger basis) of the $(2 j+1)$-dimensional space $V$ which carries the irrep $\mathbf{D}^{(j)}$ of $\mathbf{S U}(2)$. We refer to this basis as a standard basis. An orthonormal basis carrying $\mathbf{D}^{(j) *}$ is a contrastandard basis. The matrix $\mathbf{Y}$ which 
transforms a contrastandard into a standard basis is

$$
\mathbf{Y}=\left(\begin{array}{cc}
0 & -1 \\
1 & 0
\end{array}\right)
$$

This matrix has the desired property for the defining irrep of SU(2), since it can be verified by matrix multiplication and the form (4) of $\mathbf{U}$ that

$$
\mathbf{Y} \mathbf{U}^{*} \mathbf{Y}^{-1}=\mathbf{U}
$$

The matrix $\mathbf{Y}$ represents an active rotation around the $y$-axis $\hat{e}_{y}$ over $\pi$. Indeed, from equation (5) we find

$$
\begin{aligned}
\hat{U} & \left(\hat{e}_{y}, \pi\right)(|\alpha\rangle,|\beta\rangle) \\
& =(|\alpha\rangle,|\beta\rangle) \mathbf{U}\left(\hat{e}_{y}, \pi\right) \\
& =(|\alpha\rangle,|\beta\rangle)\left[\mathbf{1} \cos \frac{\pi}{2}-\mathrm{i} \sigma_{y} \sin \frac{\pi}{2}\right] \\
& =(|\alpha\rangle,|\beta\rangle)\left(\begin{array}{cc}
0 & -1 \\
1 & 0
\end{array}\right) \equiv(|\alpha\rangle,|\beta\rangle) \mathbf{Y} .
\end{aligned}
$$

Since by equation (37) $\mathbf{Y}$ maps a standard basis onto a contrastandard basis, it is often referred to as the $\mathrm{SU}(2)$ metric tensor. In order to find the $\mathrm{SU}(2)$ metric tensor for general $j$, we define a contrastandard set of boson operators. We recall the transformation rule equation (25) and define $\hat{Y}:=U\left(\hat{e}_{y}, \pi\right) \in \mathrm{SU}(2)$. Following common usage in tensor analysis, we distinguish upper and lower indices and define $\left(b^{1}\right)^{\dagger}$ and $\left(b^{2}\right)^{\dagger}$ by

$$
\left(\left(b^{1}\right)^{\dagger},\left(b^{2}\right)^{\dagger}\right):=\hat{Y}\left(b_{1}^{\dagger}, b_{2}^{\dagger}\right) \hat{Y}^{\dagger}=\left(b_{1}^{\dagger}, b_{2}^{\dagger}\right) \mathbf{Y}=\left(b_{2}^{\dagger},-b_{1}^{\dagger}\right) .
$$

Then the basis

$$
|\overline{j m}\rangle:=\frac{\left(b^{1 \dagger}\right)^{j+m}\left(b^{2 \dagger}\right)^{j-m}}{\sqrt{(j+m) !(j-m) !}}|0\rangle
$$

is contrastandard, because

(i) $\left(b^{1}\right)^{\dagger}$ and $\left(b^{2}\right)^{\dagger}$ carry $\mathbf{U}^{*}$,

(ii) $|\overline{j m}\rangle$ is the same polynomial in $\left(b^{1}\right)^{\dagger}$ and $\left(b^{2}\right)^{\dagger}$ as $|j m\rangle$ is in $b_{1}^{\dagger}$ and $b_{2}^{\dagger}(c f$. (18)),

(iii) the vectors $|j m\rangle$ carry $\mathbf{D}^{(j)}\left(c f\right.$. (34)) and hence the $|\overline{j m}\rangle$ carry $\overline{\mathbf{D}}^{(j)}$.

Using $\hat{Y}^{\dagger}|0\rangle=|0\rangle$ (cf. (22)), we find from equations (39) and (40) that

$$
|\overline{j m}\rangle=\hat{Y} \frac{\left(b_{1}^{\dagger}\right)^{j+m}\left(b_{2}^{\dagger}\right)^{j-m}}{\sqrt{(j+m) !(j-m) !}}|0\rangle=\hat{Y}|j m\rangle,
$$

as well as

$$
|\overline{j m}\rangle=(-1)^{j-m} \frac{\left(b_{1}^{\dagger}\right)^{j-m}\left(b_{2}^{\dagger}\right)^{j+m}}{(j+m) !(j-m) !}|0\rangle=(-1)^{j-m}|j,-m\rangle,
$$

which imply the following important relation

$$
|\overline{j m}\rangle=\hat{Y}|j m\rangle=(-1)^{j-m}|j,-m\rangle .
$$


Wigner [22] introduced the SU(2) metric tensor as the tensor that transforms a standard to a contrastandard basis vector:

$$
|\overline{j m}\rangle=\sum_{m^{\prime}}\left|j m^{\prime}\right\rangle\left(\begin{array}{c}
j \\
m^{\prime} m
\end{array}\right)
$$

where the tensor elements, referred to as the Wigner 1jm-symbols, have the following explicit form

$$
\left(\begin{array}{c}
j \\
m^{\prime} m
\end{array}\right):=D^{(j)}(\mathbf{Y})_{m^{\prime} m}=(-1)^{j-m} \delta_{m^{\prime},-m},
$$

as can be verified using equation (42) and upon noting that $\mathbf{D}^{(j)}(\mathbf{Y})$ is the matrix of $\hat{Y}$ in the basis $|j m\rangle$. Since SU(2) has "double group" character, i.e.,

$$
\mathbf{D}^{(j)}(\mathbf{Y}) \mathbf{D}^{(j)}(\mathbf{Y})=(-1)^{2 j} \mathbf{1}^{(j)},
$$

or explicitly,

$$
\sum_{m^{\prime}}\left(\begin{array}{c}
j \\
m m^{\prime}
\end{array}\right)\left(\begin{array}{c}
j \\
m^{\prime} \tilde{m}
\end{array}\right)=(-1)^{2 j} \delta_{m \tilde{m}}
$$

(in the case of half-integer $j$ a rotation by $2 \pi$ is minus the identity), we find that

$$
\left(\begin{array}{c}
j \\
m^{\prime} m
\end{array}\right)=(-1)^{2 j}\left(\begin{array}{c}
j \\
m m^{\prime}
\end{array}\right)
$$

so that also

$$
\left(\begin{array}{c}
j \\
m m^{\prime}
\end{array}\right)=(-1)^{j+m} \delta_{m,-m^{\prime}}
$$

This antisymmetry of the SU(2) metric tensor for half-integer $j$ is the source of unpleasant phase factors, of which we shall keep track by orienting the lines in the diagrams introduced later.

\subsection{Time reversal}

It is of interest to relate the operator $\hat{Y}$ to the operation of time reversal. We define the timereversal operator $\vartheta$ by its action on the dynamical variables position $\vec{q}$, linear momentum $\vec{p}$, and spin $\vec{s}$ (the vector operator with components defined in equation (3)):

$$
\begin{aligned}
& \vartheta \vec{q} \vartheta^{\dagger}=\vec{q}, \\
& \vartheta \vec{p} \vartheta^{\dagger}=-\vec{p}, \\
& \vartheta \vec{s} \vartheta^{\dagger}=-\vec{s}, \\
& \langle\vartheta \psi \mid \vartheta \phi\rangle\langle\vartheta \phi \mid \vartheta \psi\rangle=\langle\psi \mid \phi\rangle\langle\phi \mid \psi\rangle .
\end{aligned}
$$

Note that the requirements (47a) and (47b) imply that the orbital angular momentum $\vec{q} \times \vec{p}$ is antisymmetric under time reversal. Condition $(47 \mathrm{c})$ for the spin angular momentum is introduced by analogy. Finally, we require that Born's transition probability is left invariant by $\vartheta$, i.e., condition (47d). Wigner [8] proved that this last requirement implies that $\vartheta$ is unitary and either linear or antilinear. (Note that the sufficiency of this requirement is immediately obvious.) Considering the angular momentum commutation relations we find 
two possibilities

$$
\vartheta\left[\hat{J}_{j}, \hat{J}_{k}\right] \vartheta^{\dagger}=\sum_{l} \vartheta\left(\mathrm{i} \varepsilon_{j k l} \hat{J}_{l}\right) \vartheta^{\dagger}= \pm \mathrm{i} \sum_{l} \varepsilon_{j k l} \vartheta \hat{J}_{l} \vartheta^{\dagger}=\mp \mathrm{i} \sum_{l} \varepsilon_{j k l} \hat{J}_{l},
$$

where the upper sign holds if $\vartheta$ is linear and the lower if $\vartheta$ is antilinear. Since, on the other hand,

$$
\vartheta\left[\hat{J}_{j}, \hat{J}_{k}\right] \vartheta^{\dagger}=\left[\vartheta \hat{J}_{j} \vartheta^{\dagger}, \vartheta \hat{J}_{k} \vartheta^{\dagger}\right]=\left[-\hat{J}_{j},-\hat{J}_{k}\right]=\mathrm{i} \sum_{l} \varepsilon_{j k l} \hat{J}_{l},
$$

it follows that $\vartheta$ is antilinear, and in view of equation (47d) $\vartheta$ is antiunitary. Recall that an antiunitary operator satisfies the following turnover rule

$$
\langle\vartheta \psi \mid \vartheta \phi\rangle=\langle\phi \mid \psi\rangle=\langle\psi \mid \phi\rangle^{*},
$$

and from $\vartheta \vartheta^{\dagger}=1$,

$$
\left\langle\vartheta^{\dagger} \psi \mid \phi\right\rangle=\langle\psi \mid \vartheta \phi\rangle^{*} .
$$

Time reversal is one of the fundamental symmetries observed in nature. To date there is no experimental evidence that casts doubt on the general validity of this symmetry for electromagnetic interactions. In a time-independent formalism, the name of this symmetry may be somewhat puzzling. We notice, however, that classically linear momentum changes sign under time reversal, while position does not. These basic symmetries are preserved in the quantum mechanical definition of $\vartheta$. The reader will note that a simple sign change of the time variable, $t \rightarrow-t$, will not provide a satisfactory definition of time reversal in quantum mechanics, since this would also change the sign of the energy, as follows immediately from the time dependent Schrödinger equation. In fact, the requirement that the energy is conserved is consistent with the antilinearity of the time-reversal operator.

It is easily verified that the operators $\mathcal{J}_{k}$ in equations (28a)-(28c) satisfy $\vartheta \mathcal{J}_{k} \vartheta^{\dagger}=-\mathcal{J}_{k}$ if we assume

$$
\vartheta\left(b_{1}^{\dagger}, b_{2}^{\dagger}\right) \vartheta^{\dagger}=\mathrm{e}^{\mathrm{i} \phi}\left(b_{1}^{\dagger}, b_{2}^{\dagger}\right) \mathbf{Y} .
$$

Here $\mathrm{e}^{\mathrm{i} \phi}$ is an overall phase factor; below we shall argue that we can make the choice $\phi=0$. The matrix $\mathbf{Y}$ is given in equation (36).

We next observe that the time-reversal operator commutes with the elements of SU(2). This can be readily seen by the use of the exponential representation (7) of a general element $\hat{U} \in \mathrm{SU}(2)$. Using the antilinearity of $\vartheta$ we have for the $p$ th term in the expansion of the exponential that

$$
\vartheta[-\mathrm{i} \varphi \boldsymbol{\sigma} \cdot \vec{n}]^{p} \vartheta^{\dagger}=[-\mathrm{i} \varphi \boldsymbol{\sigma} \cdot \vec{n}]^{p} .
$$

From this the commutation of $\hat{U}$ and $\vartheta$ follows. From the antilinearity of $\vartheta$ and the commutation rule $[\hat{U}, \vartheta]=0$ just found, we can immediately conclude that $\{\vartheta|j m\rangle\}$ forms a contrastandard basis. Indeed, applying $\vartheta$ to the defining relation

$$
\hat{U}|j m\rangle=\sum_{m^{\prime}} D^{(j)}(U)_{m^{\prime} m}\left|j m^{\prime}\right\rangle,
$$

yields

$$
\vartheta \hat{U}|j m\rangle=\hat{U} \vartheta|j m\rangle=\sum_{m^{\prime}} D^{(j)}(U)_{m^{\prime} m}^{*} \vartheta\left|j m^{\prime}\right\rangle .
$$


Hence, $\hat{Y}|j m\rangle$ and $\vartheta|j m\rangle$ span the very same irrep of SU(2). From Schur's lemma and the orthonormality of the basis used follows that

$$
\hat{Y}|j m\rangle=\mathrm{e}^{\mathrm{i} \phi_{j}} \vartheta|j m\rangle,
$$

where the phase factor does not depend on $m$. Thus, a natural choice for the phase of $|j m\rangle$ is such that $\exp \left[\mathrm{i} \phi_{j}\right]$ is unity, ${ }^{2}$ so that we can write, using equation (42),

$$
\vartheta|j m\rangle \equiv|\vartheta(j m)|=(-1)^{j-m}|j,-m\rangle .
$$

Although $\hat{Y}$ and $\vartheta$ have now the same effect on $|j m\rangle$, they are not identical operators, since the one is linear and the other antilinear. By introducing the complex conjugation operator $K_{0}$ we can find an operator relationship between $Y$ and $\vartheta$. We define $K_{0}$ by

$$
K_{0}\left(c|\psi\rangle+c^{\prime}\left|\psi^{\prime}\right\rangle\right):=c^{*}|\psi\rangle+c^{\prime *}\left|\psi^{\prime}\right\rangle
$$

so that it is antilinear. We can then write that

$$
\vartheta=\hat{Y} K_{0}
$$

where $\hat{Y}$ represents a rotation by $\pi$ around the $y$-axis, see equation (38). Since $K_{0}^{2}=1$, so that

$$
\vartheta^{2}|j m\rangle=Y^{2}|j m\rangle=(-1)^{2 j}|j m\rangle,
$$

we find that for half-integer $j$ neither $\hat{Y}$ nor $\vartheta$ are involutory. ${ }^{3}$

We wish to end this section by pointing out that the bras $\{\langle j m|\}$ also form a contrastandard basis of $\mathrm{SU}(2)$, i.e.,

$$
\langle j m| \hat{U}^{\dagger}=\langle\hat{U}(j m)|=\sum_{m^{\prime}} D^{(j)}(U)_{m^{\prime} m}^{*}\left\langle j m^{\prime}\right| .
$$

We must emphasize, however, that the bra vectors span a different space (the dual space) than the ket vectors, so that there can be no linear relationship relating bras with kets. Furthermore, the process of Hermitian conjugation is involutory,

$$
|j m\rangle^{\dagger \dagger}=|j m\rangle \text {. }
$$

This already implies that we cannot represent $\vartheta|j m\rangle$ and $\langle j m|$ by the same diagram (see below), although both carry the very same irrep of $\mathrm{SU}(2)$.

\subsection{Clebsch-Gordan series}

To define the CG series we consider spaces $V_{j_{1}}$ and $V_{j_{2}}$ carrying standard irreps $D^{\left(j_{1}\right)}$ and $D^{\left(j_{2}\right)}$ of SU(2). The basis $\left|j_{1}, m_{1}\right\rangle, m_{1}=-j_{1}, \ldots, j_{1}$, consist of eigenfunctions of $J^{2}$ and $J_{z}$. The same holds for $\left|j_{2}, m_{2}\right\rangle$. The tensor product space $V_{j_{1}} \otimes V_{j_{2}}$ carries the reducible Kronecker product representation $D^{\left(j_{1}\right)} \otimes D^{\left(j_{2}\right)}$. Reduction of the tensor space

\footnotetext{
2 Note, however, that spherical harmonics with the widely used Condon and Shortley phase convention do not satisfy this choice, and differ by the phase factor $(-i)^{j}$ from the $|j m\rangle$ introduced here.

3 An operator $A$ is involutory if $A^{2}=1$.
} 
under SU(2) yields

$$
V_{j_{1}} \otimes V_{j_{2}}=\sum_{j=\left|j_{1}-j_{2}\right|}^{j_{1}+j_{2}} \oplus V_{j}
$$

where $V_{j}$ carries the irrep $D^{(j)}$ of SU(2). It is proved in textbooks by Lie-algebraic techniques that this reduction is multiplicity free, i.e., the irrep $D^{(j)}$ occurs once in this reduction. Also the range of $j$, indicated in equation (49) is proved in textbooks. Equation (49) constitutes the Clebsch-Gordan theorem, proved in 1869 by the German mathematician Paul Gordan [26], who used techniques from invariant theory developed by his colleague Alfred Clebsch.

Designating the orthonormal basis for $V_{j}$ by $\{|j, m\rangle\}$, we can insert the resolution of the identity in the tensor product space and write

$$
\left|j_{1} m_{1}\right\rangle \otimes\left|j_{2} m_{2}\right\rangle=\sum_{j=\left|j_{1}-j_{2}\right|}^{j_{1}+j_{2}} \sum_{m=-j}^{j}|j m\rangle\left\langle j m \mid j_{1} m_{1} ; j_{2} m_{2}\right\rangle,
$$

where we introduced the notation $\left|j_{1} m_{1}\right\rangle \otimes\left|j_{2} m_{2}\right\rangle \equiv\left|j_{1} m_{1} ; j_{2} m_{2}\right\rangle$. As it is common to refer to equation (50) as the SU(2) Clebsch-Gordan (CG) series, we will follow this practice. The inner product $\left\langle j m \mid j_{1} m_{1} ; j_{2} m_{2}\right\rangle$ is a Clebsch-Gordan (CG) coefficient. These coefficients transform an orthonormal basis of $V_{j_{1}} \otimes V_{j_{2}}$ into another one and form a unitary matrix $\mathbf{C}$, which decomposes the Kronecker product representation into irreducible blocks,

$$
\mathbf{C}\left[\mathbf{D}^{\left(j_{1}\right)}(\hat{U}) \otimes \mathbf{D}^{\left(j_{2}\right)}(\hat{U})\right] \mathbf{C}^{\dagger}=\sum_{j} \oplus \mathbf{D}^{(j)}(\hat{U}), \quad \hat{U} \in \mathrm{SU}(2) .
$$

This relationship exhibits the fact that the CG coefficients depend only on the irreps of $\mathrm{SU}(2)$ and not on a particular realization of $V_{j_{1}}$ and $V_{j_{2}}$, so that we can choose $\left|j_{1} m_{1}\right\rangle$ and $\left|j_{2} m_{2}\right\rangle$ arbitrarily. The first derivation of an algebraic formula for CG coefficients was given by Wigner in his famous 1931 book [8]. The following expression for the CG coefficients is due to Van der Waerden [27] and is the most symmetric one of the various existing forms, see, e.g., Ref. [13] for a derivation,

$$
\begin{aligned}
& \left\langle j m \mid j_{1} m_{1} ; j_{2} m_{2}\right\rangle \\
& =\delta_{m, m_{1}+m_{2} \Delta} \Delta\left(j_{1}, j_{2}, j\right) \\
& \times \sum_{t}(-1)^{t}\left[(2 j+1)\left(j_{1}+m_{1}\right) !\left(j_{1}-m_{1}\right) !\right. \\
& \left.\quad \times\left(j_{2}+m_{2}\right) !\left(j_{2}-m_{2}\right) !(j+m) !(j-m) !\right]^{1 / 2} \\
& \quad \times\left[t !\left(j_{1}+j_{2}-j-t\right) !\left(j_{1}-m_{1}-t\right) !\right. \\
& \left.\quad \times\left(j_{2}+m_{2}-t\right) !\left(j-j_{2}+m_{1}+t\right) !\left(j-j_{1}-m_{2}+t\right) !\right]^{-1},
\end{aligned}
$$

where

$$
\Delta\left(j_{1}, j_{2}, j\right) \equiv\left[\frac{\left(j_{1}+j_{2}-j\right) !\left(j_{1}-j_{2}+j\right) !\left(-j_{1}+j_{2}+j\right) !}{\left(j_{1}+j_{2}+j+1\right) !}\right]^{1 / 2},
$$

and the sum runs over all values of $t$ which do not lead to negative factorials.

The symmetry properties of the CG coefficients are

(i) $\left\langle j m \mid j_{1} m_{1} ; j_{2} m_{2}\right\rangle=\left\langle j_{1} m_{1} ; j_{2} m_{2} \mid j m\right\rangle$, 
(ii) $\left\langle j m \mid j_{1} m_{1} ; j_{2} m_{2}\right\rangle=(-1)^{j_{1}+j_{2}-j}\left\langle j,-m \mid j_{1},-m_{1} ; j_{2},-m_{2}\right\rangle$,

(iii) $\left\langle j m \mid j_{1} m_{1} ; j_{2} m_{2}\right\rangle=(-1)^{j_{1}+j_{2}-j}\left\langle j m \mid j_{2} m_{2} ; j_{1} m_{1}\right\rangle$,

(iv) $\left\langle j m \mid j_{1} m_{1} ; j_{2} m_{2}\right\rangle=\left[\frac{2 j+1}{2 j_{2}+1}\right]^{1 / 2}\left\langle j_{2} m_{2} \mid j m ; \vartheta\left(j_{1} m_{1}\right)\right\rangle$.

For $j=m=0, \mathrm{CG}$ coefficients are equal to

(v) $\left\langle 00 \mid j_{1} m_{1} ; j_{2} m_{2}\right\rangle=\delta_{j_{1} j_{2}} \delta_{m_{1},-m_{2}}(-1)^{j_{1}-m_{1}}\left(2 j_{1}+1\right)^{-1 / 2}$.

We will prove these properties:

(i) The CG coefficients are defined as an inner product in equation (50); their explicit form shows that they are real. Hence,

$$
\left\langle j m \mid j_{1} m_{1} ; j_{2} m_{2}\right\rangle=\left\langle j_{1} m_{1} ; j_{2} m_{2} \mid j m\right\rangle^{*}=\left\langle j_{1} m_{1} ; j_{2} m_{2} \mid j m\right\rangle .
$$

(ii) From the unitarity of the time reversal operator $\vartheta$ and the realness of CG coefficients follows that

$$
\begin{aligned}
& \left\langle j m \mid j_{1} m_{1} ; j_{2} m_{2}\right\rangle \\
& \quad=\left\langle j m\left|\vartheta^{\dagger} \vartheta\right| j_{1} m_{1} ; j_{2} m_{2}\right\rangle=\left\langle\vartheta(j m) \mid \vartheta\left(j_{1} m_{1}\right) ; \vartheta\left(j_{2} m_{2}\right)\right\rangle \\
& \quad=(-1)^{j+j_{1}+j_{2}}(-1)^{-\left(m+m_{1}+m_{2}\right)}\left\langle j,-m \mid j_{1},-m_{1} ; j_{2},-m_{2}\right\rangle .
\end{aligned}
$$

Since $m=m_{1}+m_{2}$ and $m$ is a half-integer if and only if $j$ is a half-integer, we find that

$$
(-1)^{-\left(m+m_{1}+m_{2}\right)}=(-1)^{-2 m}=(-1)^{-2 j} .
$$

(iii) The explicit form for CG coefficients, equation (51), is invariant with respect to a simultaneous interchange $j_{1} \leftrightarrow j_{2}$ and $m_{1} \leftrightarrow-m_{2}$, which implies that $m \rightarrow-m$ and thus

$$
\begin{aligned}
& \left\langle j m \mid j_{1} m_{1} ; j_{2} m_{2}\right\rangle \\
& \quad=\left\langle j,-m \mid j_{2},-m_{2} ; j_{1},-m_{1}\right\rangle=(-1)^{j_{1}+j_{2}-j}\left\langle j m \mid j_{2} m_{2} ; j_{1} m_{1}\right\rangle .
\end{aligned}
$$

(iv) Make simultaneous replacements $j \leftrightarrow j_{2}$ and $m \leftrightarrow-m_{2}$ in the explicit expression for CG coefficients, equation (51), and change the summation variable $t$ to $j_{1}-m_{1}-t$. Observe that except for the first factor $(2 j+1)^{1 / 2}$ and the phase $(-1)^{t}$ they leave the expression (51) invariant. Thus

$$
\begin{aligned}
\langle j m & \left|j_{1} m_{1} ; j_{2} m_{2}\right\rangle \\
= & {\left[\frac{2 j+1}{2 j_{2}+1}\right]^{1 / 2}(-1)^{j_{1}-m_{1}}\left\langle j_{2},-m_{2} \mid j_{1} m_{1} ; j,-m\right\rangle } \\
& =\left[\frac{2 j+1}{2 j_{2}+1}\right]^{1 / 2}(-1)^{j_{1}-m_{1}}\left\langle j_{2} m_{2} \mid j m ; j_{1},-m_{1}\right\rangle \\
& =\left[\frac{2 j+1}{2 j_{2}+1}\right]^{1 / 2}\left\langle j_{2} m_{2} \mid j m ; \vartheta\left(j_{1} m_{1}\right)\right\rangle .
\end{aligned}
$$

(v) Follows by applying rule (iv) to the identity

$$
\left\langle j_{1} m_{1} \mid j_{2} m_{2} ; 00\right\rangle=\delta_{j_{1} j_{2}} \delta_{m_{1} m_{2}} .
$$




\section{DIAGRAMS}

We shall now introduce the basic concepts of a graphical technique, sometimes referred to as the graphical method of spin algebras, which is designed to facilitate the manipulation of complicated expressions involving CG coefficients, their simplification and evaluation.

Our presentation will be based on the well-defined transformation properties of CG coefficients, which follow immediately from their definition as a vector space inner product, given in the preceding section. The four group-theoretical characteristics of special importance in this respect are:

(1) the SU(2) irrep to which the state belongs (i.e., its $j$-quantum number),

(2) the row of the irrep according to which the state transforms (its $m$-quantum number),

(3) the variance of the state (does the state transform according to a standard or a contrastandard irrep?),

(4) and finally, is the state a bra or a ket?

\subsection{Basic diagrammatic building blocks}

We first introduce a diagrammatic representation for various states which enter the inner products (i.e., Clebsch-Gordan coefficients) considered. We must emphasize that our graphical technique is intended to manipulate these inner products and not the states themselves. Moreover, the inner products are always real, so that the diagrams are invariant under a simultaneous Hermitian conjugation of all states.

We draw the vertices arising in the CG diagrams as half circles, and adhere to the following rules.

Convention C1. A state transforming according to a standard rep is designated by an oriented line leaving a vertex, while a state transforming according to a contrastandard rep is represented by a line entering a vertex.

CONVEntion C2. A ket is represented by a single arrow and a bra by a double arrow.

Convention C3. An $n$-fold tensor product is represented by $n$ lines attached to a single vertex. The vertex sign defines the order in the tensor product: a minus sign indicates a clockwise and a plus sign a counter clockwise reading. Note that this corresponds to the sense of rotation around an axis pointing towards the reader. Evidently, when only one line is attached to a vertex, a node sign is superfluous.

We thus have the following states:

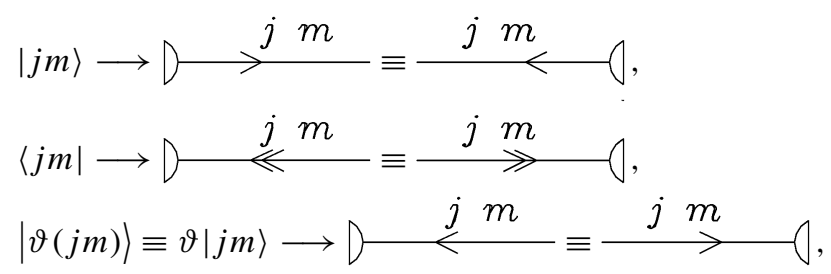




$$
\langle\vartheta(j m)| \equiv\langle j m| \vartheta^{\dagger} \longrightarrow D \longrightarrow \overbrace{}^{j m} \equiv j^{j m} \ll .
$$

For a tensor product we have

$$
\left|j_{1} m_{1} ; \ldots ; j_{n} m_{n}\right\rangle:=\left|j_{1} m_{1}\right\rangle \otimes \cdots \otimes\left|j_{n} m_{n}\right\rangle
$$

As an example of a mixed standard-contrastandard tensor we give

$$
\left|\vartheta\left(j_{1} m_{1}\right) ; j_{2} m_{2}\right\rangle:=\left(\vartheta\left|j_{1} m_{1}\right\rangle\right) \otimes\left|j_{2} m_{2}\right\rangle \rightarrow
$$

An inner product of two states is then represented as

$$
\begin{aligned}
& \left\langle j_{1} m_{1} \mid j_{2} m_{2}\right\rangle=\delta_{j_{1} j_{2}} \delta_{m_{1} m_{2}} \\
& \quad \underline{j_{1} m_{1} \gg \mid}>j_{2} m_{2}:=\underline{j_{1} m_{1} \gg \longrightarrow j_{2} m_{2}}
\end{aligned}
$$

and, for tensor product states, as

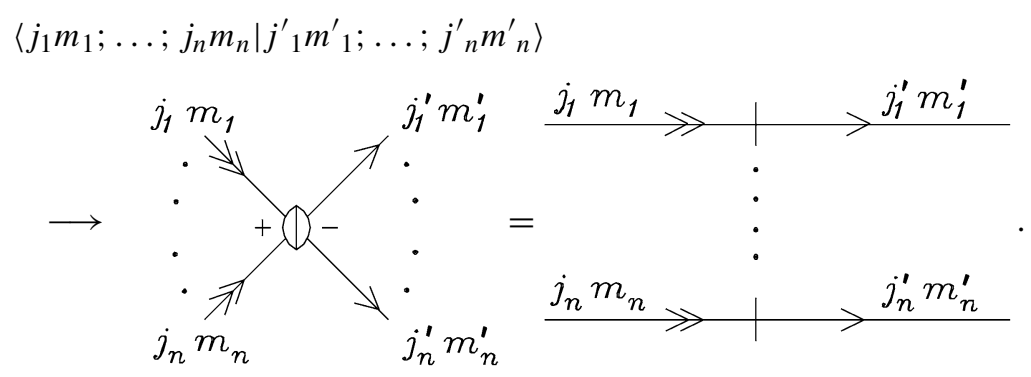

The central concept of graphical techniques is that of contraction, representing the formation of an SU(2) invariant by summing over the $m$-quantum number of a product of a standard and a contrastandard state. We introduce the following four possible prototypes for a contraction.

\section{CONVENTION C4.}

(i) Ket-bra contraction (projector):

$$
\sum_{m}|j m\rangle\langle j m|=\sum_{m} D>^{j m} \stackrel{j m}{\longrightarrow}(1=0 \quad j
$$


Since

$$
\begin{aligned}
\sum_{m}|j m\rangle\langle j m| & =\sum_{m}|j,-m\rangle\langle j,-m| \\
& =\sum_{m}(-1)^{2(j-m)}|j,-m\rangle\langle j,-m| \\
& =\sum_{m} \vartheta|j m\rangle\langle j m| \vartheta^{\dagger},
\end{aligned}
$$

we also have that

$$
\begin{aligned}
& \sum_{m} 0 \stackrel{j m}{\longrightarrow} \stackrel{j m}{\longrightarrow} \\
& =\sum_{m} D \ll^{j m} \ll^{j m}(=0) j
\end{aligned}
$$

which implies that it is consistent not to orient the contracted line.

(ii) Bra-ket contraction (inner product contraction):

$$
\begin{aligned}
2 j+1 & =\sum_{m}\langle j m \mid j m\rangle=\sum_{m}\left\langle j m\left|\vartheta^{\dagger} \vartheta\right| j m\right\rangle \\
\left.\longrightarrow \sum_{m} \gg\right\rangle^{j m,}>^{j m} & =\sum_{m} \stackrel{j m, j m}{<} \\
& ={ }^{j} .
\end{aligned}
$$

(iii) Ket-time-reversed ket contraction:

$$
\sum_{m}|\vartheta(j m)||j m\rangle \longrightarrow \sum_{m} D \stackrel{j}{\stackrel{j}{<}} \stackrel{j m}{<} \stackrel{j}{<}
$$

Since

$$
\begin{aligned}
\sum_{m}|\vartheta(j m)\rangle|j m\rangle & =\sum_{m}(-1)^{j-m}|j,-m\rangle|j m\rangle=\sum_{m}(-1)^{j+m}|j m\rangle|j,-m\rangle \\
& =(-1)^{2 j} \sum_{m}|j m\rangle|\vartheta(j m)\rangle
\end{aligned}
$$

and

$$
\sum_{m}|j m\rangle|\vartheta(j m)\rangle=0>^{j}
$$

we see that the contracted line must be oriented. At the same time we obtain the rule for arrow reversal on internal lines, namely




(iv) Bra-time-reversed bra contraction: This is essentially the Hermitian conjugate of case (iii). We can define

$$
\sum_{m}|\vartheta(j m)|\langle j m| \longrightarrow 0 \stackrel{j}{\longleftarrow}
$$

that is,



In terms of our graphical conventions we thus obtain the following four simple rules for the contraction of free (external) lines:

- Only lines which preserve the orientation can be contracted.

- Contraction of a single-arrowed line with a double-arrowed line produces an unoriented line.

- Contraction of two single-arrowed lines yields a single-arrowed line with preserved orientation.

- Contraction of two double-arrowed lines again produces a single-arrowed line, but with reversed orientation.

\subsection{Clebsch-Gordan diagrams}

We define

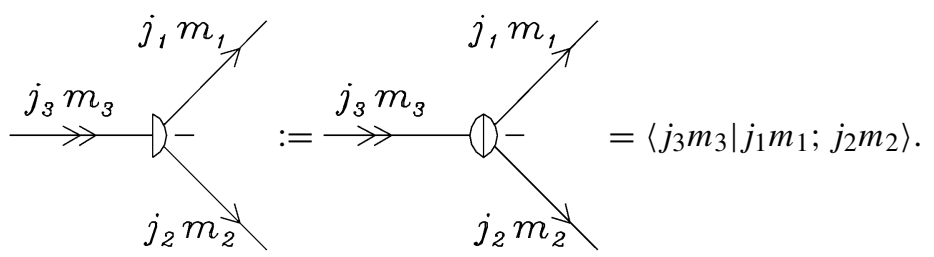

Two lines of a CG coefficient represent states which are either both in the bra or both in the ket. We refer to these lines as the non-unique lines. The third line, the unique line, is attached to the straight side of the vertex. Note that the vertex sign defines the order of the non-unique lines.

The symmetry properties of the CG coefficient (Section 2.5) are diagrammatically represented as follows (for conciseness we replace the label $j_{k} m_{k}$ by $k, k=1,2,3$ ):

(i)




(ii)

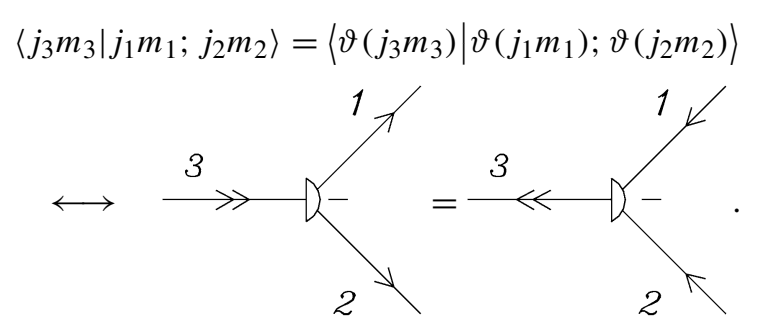

(iii) $\quad\left\langle j_{3} m_{3} \mid j_{1} m_{1} ; j_{2} m_{2}\right\rangle=(-1)^{j_{1}+j_{2}-j_{3}}\left\langle j_{3} m_{3} \mid j_{2} m_{2} ; j_{1} m_{1}\right\rangle$



(iv)

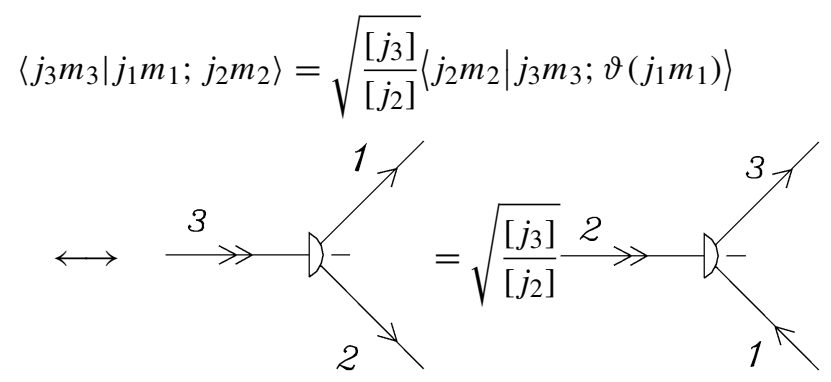

with $\left[j_{k}\right]:=2 j_{k}+1, k=1,2,3$.

(v)

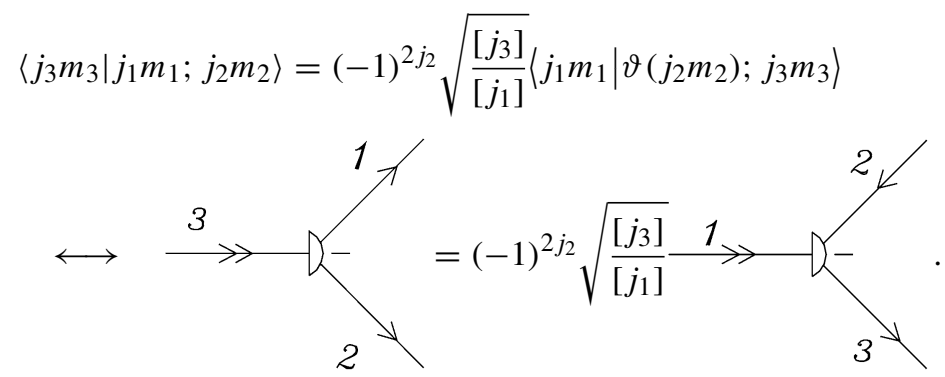

Rule (62) results from successive application of rules (60), (61) and again (60).

Since the states $|j m\rangle$ form also an orthonormal basis for the tensorial product space spanned by $\left|j_{1} m_{1} ; j_{2} m_{2}\right\rangle$, for certain fixed $j_{1}$ and $j_{2}$ and with $m_{1}=-j_{1}, \ldots, j_{1}$ and 
$m_{2}=-j_{2}, \ldots, j_{2}$, we find the first orthogonality relation

$$
\begin{gathered}
\sum_{j=\left|j_{1}-j_{2}\right|}^{j_{1}+j_{2}} \sum_{m=-j}^{j}\left\langle j_{1} m_{1} ; j_{2} m_{2} \mid j m\right\rangle\left\langle j m \mid j_{1} m_{1}^{\prime} ; j_{2} m_{2}^{\prime}\right\rangle \\
=\left\langle j_{1} m_{1} ; j_{2} m_{2} \mid j_{1} m_{1}^{\prime} ; j_{2} m_{2}^{\prime}\right\rangle=\delta_{m_{1} m_{1}^{\prime}} \delta_{m_{2} m_{2}^{\prime}},
\end{gathered}
$$

which can be represented graphically as follows:

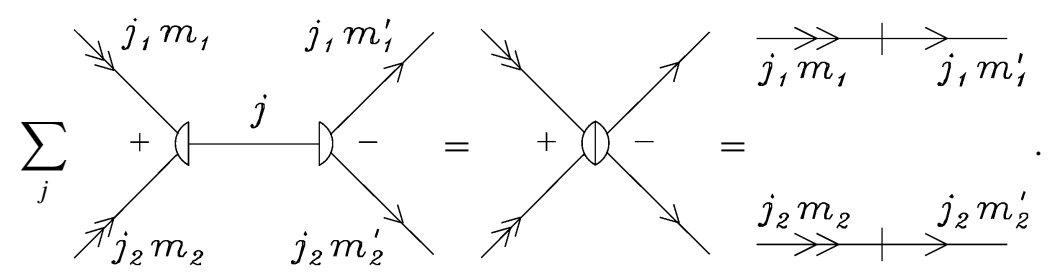

In order to obtain the second orthogonality relation, we first define a $3 j$-symbol ${ }^{4}$ or triangular delta by

$$
\left\{j, j_{1}, j_{2}\right\}= \begin{cases}1 & \text { if }\left|j_{1}-j_{2}\right| \leqslant j \leqslant j_{1}+j_{2} \\ 0 & \text { otherwise }\end{cases}
$$

We can write

$$
\begin{aligned}
& \sum_{m_{1} m_{2}}\left\langle j m \mid j_{1} m_{1} ; j_{2} m_{2}\right\rangle\left\langle j_{1} m_{1} ; j_{2} m_{2} \mid j^{\prime} m^{\prime}\right\rangle \\
& \quad=\left\{j, j_{1}, j_{2}\right\}\left\{j_{1}, j_{2}, j^{\prime}\right\}\left\langle j m \mid j^{\prime} m^{\prime}\right\rangle=\delta_{j j^{\prime}} \delta_{m m^{\prime}}\left\{j, j_{1}, j_{2}\right\},
\end{aligned}
$$

or graphically,

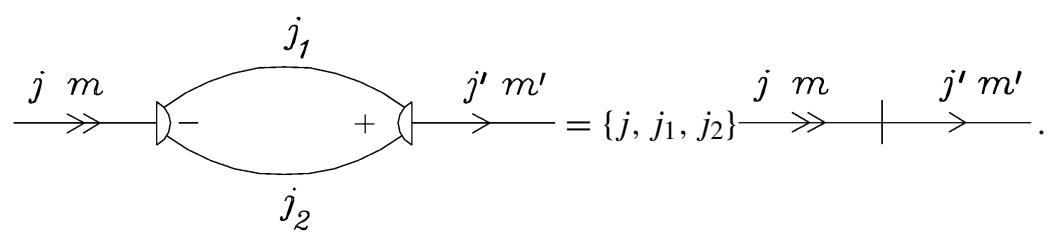

Taking $j=j^{\prime}, m=m^{\prime}$ and summing over $m$, we obtain

$$
\left\{j, j_{1}, j_{2}\right\}=[j]^{-1} \sum_{m, m_{1}, m_{2}}\left\langle j m \mid j_{1} m_{1} ; j_{2} m_{2}\right\rangle\left\langle j_{1} m_{1} ; j_{2} m_{2} \mid j m\right\rangle,
$$

so that diagrammatically $\left\{j, j_{1}, j_{2}\right\}$ can be represented by an "oyster" diagram

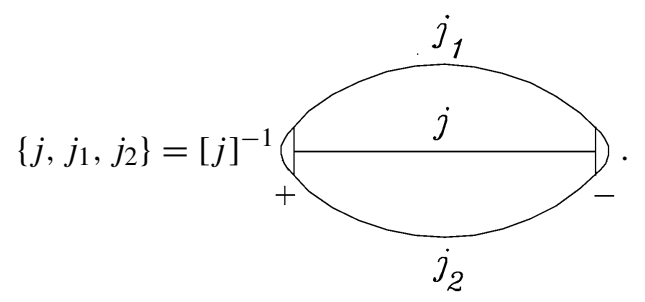

\footnotetext{
4 We wish to stress that the $3 j$-symbol must not be confused with the $3 j m$-symbol introduced below. Confusion is possible since the latter is often referred to by other authors as a $3 j$-symbol.
} 


\section{3. $1 j m$-symbol}

We have seen in Section 2.4 that the time-reversal operator transforms a standard state into a contrastandard one and vice versa. Thus, the matrix representation of $\vartheta$ plays the role of a metric tensor and we now write

$$
\left(\begin{array}{c}
j \\
m m^{\prime}
\end{array}\right)=\left\langle j m \mid \vartheta\left(j m^{\prime}\right)\right\rangle \text {. }
$$

The facts $\vartheta^{\dagger} \vartheta=1$ and $\vartheta^{2}=(-1)^{2 j}$ imply that

$$
\begin{aligned}
\left\langle j m \mid \vartheta\left(j m^{\prime}\right)\right\rangle & =\left\langle j m \mid \vartheta^{\dagger} \vartheta \vartheta\left(j m^{\prime}\right)\right\rangle=\left\langle\vartheta(j m)\left|\vartheta^{2}\left(j m^{\prime}\right)\right|^{*}\right. \\
& =(-1)^{2 j}\left\langle j m^{\prime} \mid \vartheta(j m)\right\rangle .
\end{aligned}
$$

The $1 j m$-symbols are real, since

$$
\left(\begin{array}{c}
j \\
m m^{\prime}
\end{array}\right)=(-1)^{j-m^{\prime}}\left\langle j m \mid j,-m^{\prime}\right\rangle=(-1)^{j-m^{\prime}} \delta_{m,-m^{\prime}}=(-1)^{j+m} \delta_{m,-m^{\prime}},
$$

in agreement with equations (44) and (46). Clearly the identity (66) is equivalent to the antisymmetry (equation (45)) of the metric tensor (equation (65)).

Diagrammatically we can represent the $1 \mathrm{jm}$-symbol in one of the following ways as equation (66) implies:

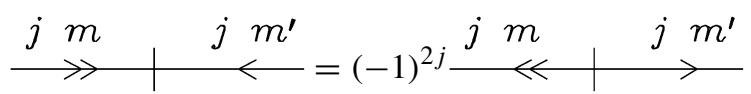

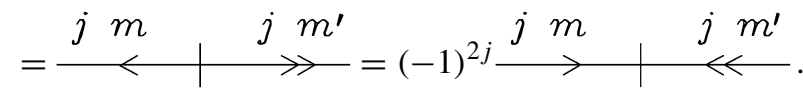

Algebraic equivalents of these diagrams are, respectively,

$$
\begin{aligned}
\left\langle j m \mid \vartheta\left(j m^{\prime}\right)\right\rangle & =(-1)^{2 j}\left\langle\vartheta(j m) \mid j m^{\prime}\right\rangle \\
& =\left\langle\vartheta\left(j m^{\prime}\right) \mid j m\right\rangle=(-1)^{2 j}\left\langle j m^{\prime} \mid \vartheta(j m)\right\rangle,
\end{aligned}
$$

and, as we just saw,

$$
\left\langle j m \mid \vartheta\left(j m^{\prime}\right)\right\rangle=(-1)^{j-m^{\prime}} \delta_{m,-m^{\prime}}=(-1)^{j+m} \delta_{m,-m^{\prime}} .
$$

The transformation of a ket to a contrastandard one, $c f$. equation (43),

$$
\vartheta|j m\rangle=\sum_{m^{\prime}}\left|j m^{\prime}\right\rangle\left(\begin{array}{c}
j \\
m^{\prime} m
\end{array}\right)=(-1)^{j-m}|j,-m\rangle,
$$

reads graphically

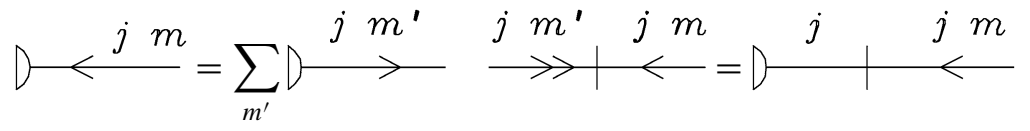

which confirms that contraction yields a projection operator on the space $V_{j}$ with its restriction $\hat{1}_{j}$ being the unit operator in $V_{j}$,

$$
0-j=\sum_{m^{\prime}}\left|j m^{\prime}\right\rangle\left\langle j m^{\prime}\right|=\hat{1}_{j} .
$$


The $1 \mathrm{jm}$-symbol is also closely related to the coupling of two angular momenta to a zero momentum. The latter will be represented by a dashed line. Clearly, if one of the coupled momenta vanishes, we have that

$$
\left\langle j m \mid j^{\prime} m^{\prime} ; 00\right\rangle=\left\langle j m \mid 00 ; j^{\prime} m^{\prime}\right\rangle=\left\langle j m \mid j^{\prime} m^{\prime}\right\rangle=\delta_{j j^{\prime}} \delta_{m m^{\prime}}
$$

or

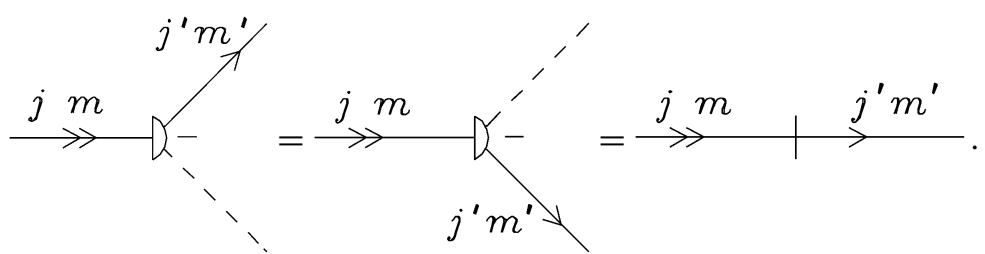

Recalling the symmetry relations given in Section 2.5 , we can write

$$
\begin{aligned}
\left\langle 00 \mid j m ; j^{\prime} m^{\prime}\right\rangle & =\left\langle j m ; j^{\prime} m^{\prime} \mid 00\right\rangle=\left[j^{\prime}\right]^{-1 / 2}\left\langle j^{\prime} m^{\prime} \mid 00 ; \vartheta(j m)\right\rangle \\
& =\left[j^{\prime}\right]^{-1 / 2}\left\langle j^{\prime} m^{\prime} \mid \vartheta(j m)\right\rangle=\delta_{j j^{\prime}}[j]^{-1 / 2}\left(\begin{array}{c}
j \\
m^{\prime} m
\end{array}\right) \\
& =\delta_{j j^{\prime}} \delta_{m,-m^{\prime}}(-1)^{j-m}[j]^{-1 / 2},
\end{aligned}
$$

which can be represented graphically as

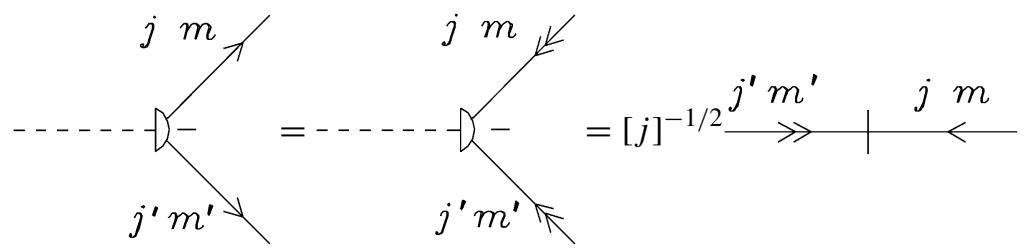

or any one of the other equivalent forms of the $1 \mathrm{jm}$-symbol shown in equation (67). This implies that the zero angular momentum line can always be eliminated from our diagrams by the use of rule (72) or (74). We do not present other possibilities, since we shall give the general rule below, when we introduce a more symmetric representation of the coupling coefficients.

\subsection{Generalized Clebsch-Gordan coefficients}

When coupling more than two angular momenta to resulting $j$ and $m$ we are faced with a multiplicity problem, since we get generally more than one linearly independent state for a given $j$ and $m$. To achieve a unique labeling of states we thus need additional labels; these can be supplied through quantum numbers characterizing intermediate couplings. We employ the genealogy (i.e., the set of intermediate coupling $j$-quantum numbers) with respect to a certain coupling scheme (the sequence of pairwise couplings) to resolve the multiplicity problem. Designating the genealogy by $(\alpha)$ we thus define the generalized $C G$ series as

$$
|(\alpha) j m\rangle=\sum_{m_{1}, \ldots, m_{n}}\left|j_{1} m_{1} ; \ldots ; j_{n} m_{n}\right\rangle\left\langle j_{1} m_{1} ; \ldots ; j_{n} m_{n} \mid(\alpha) j m\right\rangle,
$$

where $\left\langle j_{1} m_{1} ; \ldots ; j_{n} m_{n} \mid(\alpha) j m\right\rangle$ is a generalized $C G$ coefficient. 
To be more specific we consider the case of three angular momenta and the coupling scheme $\left(\left(j_{1} j_{2}\right) j_{3}\right)$. A state with an intermediate quantum number $j_{12}$ is given by the generalized CG series

$$
\begin{aligned}
& \left|\left(\left(j_{1} j_{2}\right) j_{12} j_{3}\right) j m\right\rangle \\
& \quad=\sum_{m_{1}, m_{2}, m_{3}}\left|j_{1} m_{1} ; j_{2} m_{2} ; j_{3} m_{3}\right\rangle\left\langle j_{1} m_{1} ; j_{2} m_{2} ; j_{3} m_{3} \mid\left(\left(j_{1} j_{2}\right) j_{12} j_{3}\right) j m\right\rangle .
\end{aligned}
$$

By definition

$$
\begin{aligned}
& \left\langle j_{1} m_{1} ; j_{2} m_{2} ; j_{3} m_{3} \mid\left(\left(j_{1} j_{2}\right) j_{12} j_{3}\right) j m\right\rangle \\
& \quad=\sum_{m_{12}}\left\langle j_{1} m_{1} ; j_{2} m_{2} \mid j_{12} m_{12}\right\rangle\left\langle j_{12} m_{12} ; j_{3} m_{3} \mid j m\right\rangle
\end{aligned}
$$

i.e., we first couple $j_{1}$ and $j_{2}$ to the angular momentum $j_{12}$, and then the $\left|j_{12} m_{12}\right\rangle$ is coupled with $\left|j_{3} m_{3}\right\rangle$ to the final $j$ and $m$. When we replace for simplicity the labels $j_{k}, m_{k}$ by $k$, we have diagrammatically

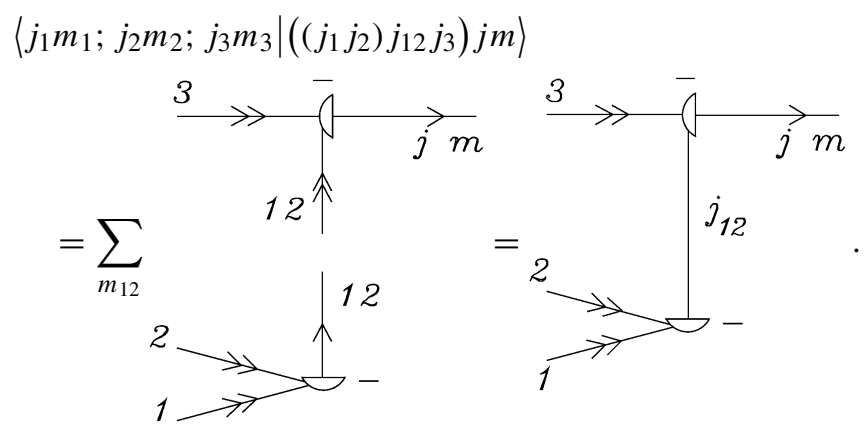

Another possible coupling scheme is, for example, $\left(j_{1}\left(j_{2} j_{3}\right)\right)$, which yields the following CG coefficient

$$
\left\langle j_{1} m_{1} ; j_{2} m_{2} ; j_{3} m_{3} \mid\left(j_{1}\left(j_{2} j_{3}\right) j_{23}\right) j m\right\rangle=\underbrace{-}_{j_{23}}
$$

In the general case, when coupling $N$ angular momenta, the generalized CG coefficient will be diagrammatically represented by a tree with $N+1$ external lines and $N-1$ CG type vertices, whose internal structure reflects the chosen coupling scheme. Since the internal (contracted) lines are invariant, it is not difficult to see that the SU(2) transformation properties of these quantities are fully determined by the external lines. We will elaborate on this point in Section 4, where we shall exploit this fact by deriving various rules for manipulation of diagrams. First, however, we shall consider another diagrammatic representation of CG-like quantities, which better reflects their inherent symmetry properties. 


\section{5. $3 \mathrm{jm}$-symbols}

The symmetry properties of CG coefficients (see Section 2.5) reflect the asymmetry of the basic coupling problem and, consequently, have quite an awkward diagrammatic representation ( $c f$. Section 3.2). We shall thus introduce quantities which are proportional to CG coefficients but have much nicer symmetry properties, namely the so-called $3 j$-symbols [22]. To avoid confusion with the triangular deltas $\left\{j_{1}, j_{2}, j_{3}\right\}$ we refer to them as $3 j m$-symbols.

We shall introduce the $3 \mathrm{jm}$-symbols by considering the coupling of three angular momenta to zero momentum. We have just seen that there are at least two possible coupling schemes and corresponding generalized CG coefficients, equations (77) and (78). If we set $j=m=0$ and remove the zero line, these coefficients become

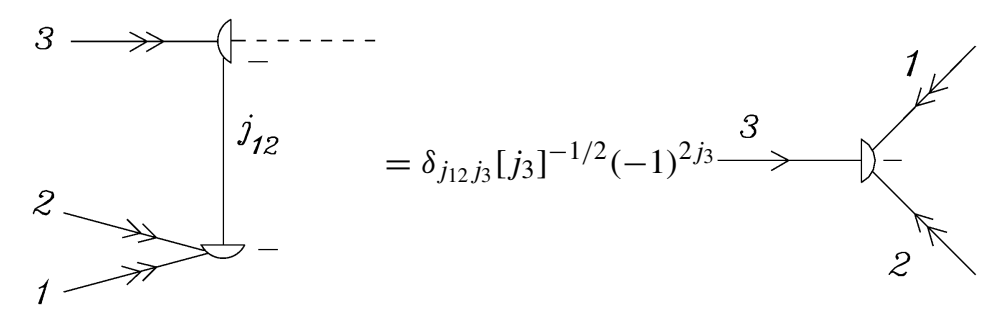

and

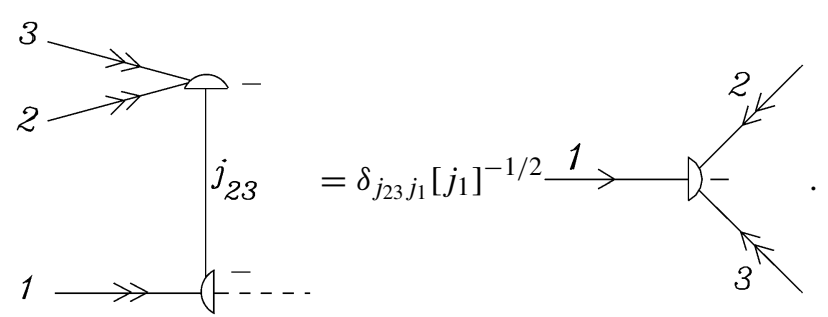

Considering the diagram on the right-hand side of equation (79), applying the symmetry property (v), equation (62), and the relation

$$
\left\langle\vartheta\left(j_{2} m_{2}\right) ; \vartheta\left(j_{3} m_{3}\right) \mid j_{1} m_{1}\right\rangle=(-1)^{2 j_{2}+2 j_{3}}\left\langle j_{2} m_{2} ; j_{3} m_{3} \mid \vartheta\left(j_{1} m_{1}\right)\right\rangle,
$$

which follows immediately from property (ii), equations (59) and (48), i.e.,

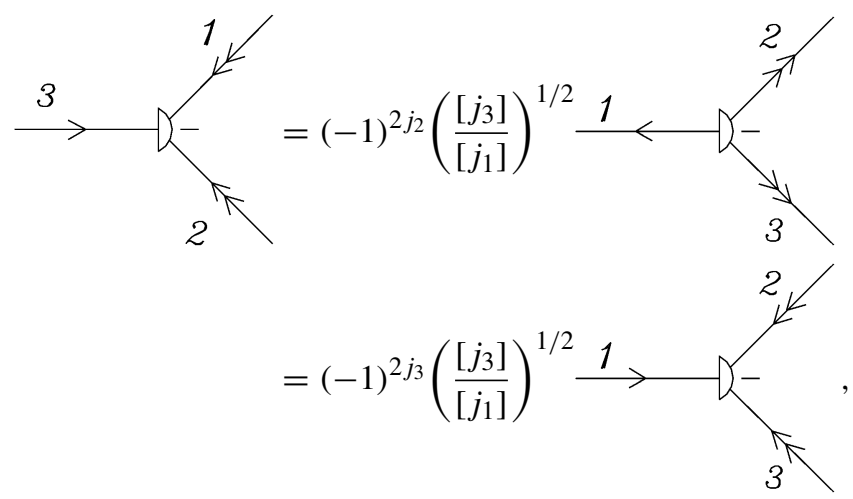


we see that both resulting generalized CG coefficients, equations (79) and (80), are identical. We obtain a unique state

$$
\left|\left(j_{1} j_{2} j_{3}\right) 00\right\rangle:=\left|\left(\left(j_{1} j_{2}\right) j_{12} j_{3}\right) 00\right\rangle \equiv\left|\left(j_{1}\left(j_{2} j_{3}\right) j_{23}\right) 00\right\rangle,
$$

and can define the $3 j m$-symbol by the relation

$$
\left\langle\left(j_{1} j_{2} j_{3}\right) 00\right|=:(-1)^{j_{1}-j_{2}+j_{3}} \sum_{m_{1} m_{2} m_{2}}\left\langle j_{1} m_{1} ; j_{2} m_{2} ; j_{3} m_{3}\right|\left(\begin{array}{ccc}
j_{1} & j_{2} & j_{3} \\
m_{1} & m_{2} & m_{3}
\end{array}\right) .
$$

Since the left-hand side of this equation is $\operatorname{SU}(2)$ invariant $(j=0)$ and the tensor product $\left\langle j_{1} m_{1} ; j_{2} m_{2} ; j_{3} m_{3}\right|$ transforms as a triple Kronecker product of contrastandard irreps, the $3 \mathrm{jm}$-symbol transforms as a triple product of standard irreps. Indeed, the defining equation (82) gives for $U \in \mathrm{SU}(2)$

$$
\begin{aligned}
&(-1)^{j_{1}-j_{2}+j_{3}}\left(\begin{array}{ccc}
j_{1} & j_{2} & j_{3} \\
m_{1} & m_{2} & m_{3}
\end{array}\right) \\
&=\left\langle\left(j_{1} j_{2} j_{3}\right) 00 \mid j_{1} m_{1} ; j_{2} m_{2} ; j_{3} m_{3}\right\rangle \\
&=\left\langle\left(j_{1} j_{2} j_{3}\right) 00\left|U^{\dagger} U\right| j_{1} m_{1} ; j_{2} m_{2} ; j_{3} m_{3}\right\rangle \\
&=(-1)^{j_{1}-j_{2}+j_{3}} \\
& \times \sum_{\mu_{1} \mu_{2} \mu_{3}}\left(\begin{array}{ccc}
j_{1} & j_{2} & j_{3} \\
\mu_{1} & \mu_{2} & \mu_{3}
\end{array}\right) D^{\left(j_{1}\right)}(U)_{\mu_{1} m_{1}} D^{\left(j_{2}\right)}(U)_{\mu_{2} m_{2}} D^{\left(j_{3}\right)}(U)_{\mu_{3} m_{3}} .
\end{aligned}
$$

For the graphical representation of the $3 j m$-symbol we realize that the three angular momenta appear in the ket and are standard, so that they must be represented by singlearrowed outgoing lines. Accordingly we set ${ }^{5}$

$$
\left(\begin{array}{ccc}
j_{1} & j_{2} & j_{3} \\
m_{1} & m_{2} & m_{3}
\end{array}\right)=: \overbrace{j_{1} m_{1}}^{j_{j} m_{3}},
$$

the minus sign at the vertex indicating a clockwise reading in accordance with our conventions.

The $3 j m$-symbol times $(-1)^{j_{1}-j_{2}+j_{3}}$, as defined by equation (82), is equal to the generalized CG coefficient on the left-hand side of equations (79) and (80). The second of these equations states that

$$
(-1)^{j_{1}-j_{2}+j_{3}}\left(\begin{array}{ccc}
j_{1} & j_{2} & j_{3} \\
m_{1} & m_{2} & m_{3}
\end{array}\right)=\left[j_{1}\right]^{-1 / 2}\left\langle j_{2} m_{2} ; j_{3} m_{3} \mid \vartheta\left(j_{1} m_{1}\right)\right\rangle,
$$

which implies the following relationship between the $3 \mathrm{jm}$ - and $\mathrm{CG}$ coefficients

$$
\left(\begin{array}{ccc}
j_{1} & j_{2} & j_{3} \\
m_{1} & m_{2} & m_{3}
\end{array}\right)=\left[j_{1}\right]^{-1 / 2}(-1)^{j_{2}-j_{3}-m_{1}}\left\langle j_{1},-m_{1} \mid j_{2} m_{2} ; j_{3} m_{3}\right\rangle .
$$

This relation is often used as the definition of a $3 \mathrm{jm}$-symbol.

5 When no confusion can arise, we shall again replace the label $j_{k} m_{k}$ simply by $k$ and for the contracted lines $j_{k}$ by $k$. 
Let us now investigate some basic properties of the $3 \mathrm{jm}$-symbol. We see immediately from equation (85) that the $3 j m$-symbol vanishes unless

$$
m_{1}+m_{2}+m_{3}=0 .
$$

It is also easy to see that it is symmetric or antisymmetric under the permutation of its columns. Indeed, using the symmetry property (iii) of CG coefficients (equation (60)) together with equation (84) we find that

$$
\left(\begin{array}{ccc}
j_{1} & j_{2} & j_{3} \\
m_{1} & m_{2} & m_{3}
\end{array}\right)=(-1)^{j_{1}+j_{2}+j_{3}}\left(\begin{array}{ccc}
j_{1} & j_{3} & j_{2} \\
m_{1} & m_{3} & m_{2}
\end{array}\right) .
$$

Further, a $3 j m$-symbol is invariant under a cyclic permutation of all three columns,

$$
\left(\begin{array}{ccc}
j_{2} & j_{3} & j_{1} \\
m_{2} & m_{3} & m_{1}
\end{array}\right)=\left(\begin{array}{ccc}
j_{1} & j_{2} & j_{3} \\
m_{1} & m_{2} & m_{3}
\end{array}\right)
$$

as can be seen easily by applying the cyclic permutation (1 23 ) to equation (79),

$$
(-1)^{j_{2}-j_{3}+j_{1}}\left(\begin{array}{ccc}
j_{2} & j_{3} & j_{1} \\
m_{2} & m_{3} & m_{1}
\end{array}\right)=(-1)^{2 j_{1}}\left[j_{1}\right]^{-1 / 2} \longrightarrow
$$

applying equation (84) to the right-hand side and observing that $j_{1}-j_{2}+j_{3}$ is integer, so that $(-1)^{2 j_{1}-2 j_{2}+2 j_{3}}=1$. Since the permutations (123) and (23) generate all 3! permutations of the three columns, we have found the general rule:

A $3 \mathrm{jm}$-symbol is invariant under an even permutation of its columns and obtains the phase factor $(-)^{j_{1}+j_{2}+j_{3}}$ upon an odd permutation of its columns.

The invariance of a $3 j m$-symbol with respect to cyclic permutations is reflected in the three-fold symmetry of the $3 \mathrm{jm}$-diagram, while the relationship (87) leads to the rule for the reversal of vertex sign:

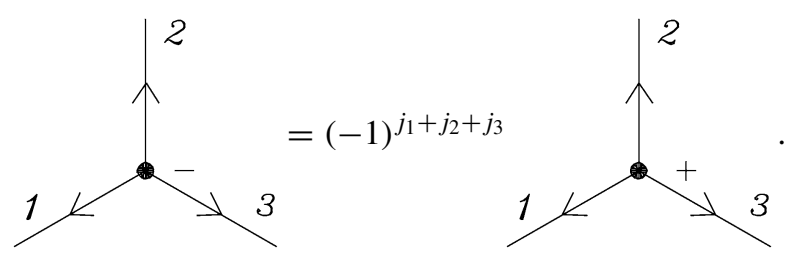

It is also useful to define mixed standard-contrastandard $3 \mathrm{jm}$-symbols, such as, for example,

$$
\left(\begin{array}{ccc}
\mu_{1} & j_{2} & j_{3} \\
j_{1} & m_{2} & m_{3}
\end{array}\right):=(-1)^{j_{1}-j_{2}+j_{3}}\left\langle\left(j_{1} j_{2} j_{3}\right) 00 \mid \vartheta\left(j_{1} \mu_{1}\right) ; j_{2} m_{2} ; j_{3} m_{3}\right\rangle .
$$

This symbol obviously transforms as $D^{\left(j_{1}\right) *} \otimes D^{\left(j_{2}\right)} \otimes D^{\left(j_{3}\right)}$, which we indicate by placing the magnetic quantum number $\mu_{1}$ in the upper row of the $3 j m$-symbol. It is easy to see that we can lower and raise the magnetic quantum number using the $1 \mathrm{jm}$-symbol. Indeed, 
applying equation (69) we obtain

$$
\begin{aligned}
\left(\begin{array}{ccc}
\mu_{1} & j_{2} & j_{3} \\
j_{1} & m_{2} & m_{3}
\end{array}\right) & =(-1)^{j_{1}-j_{2}+j_{3}} \sum_{m_{1}}\left\langle\left(j_{1} j_{2} j_{3}\right) 00 \mid j_{1} m_{1} ; j_{2} m_{2} ; j_{3} m_{3}\right\rangle\left(\begin{array}{c}
j_{1} \\
m_{1} \mu_{1}
\end{array}\right) \\
& =\sum_{m_{1}}\left(\begin{array}{ccc}
j_{1} & j_{2} & j_{3} \\
m_{1} & m_{2} & m_{3}
\end{array}\right)\left(\begin{array}{c}
j_{1} \\
m_{1} \mu_{1}
\end{array}\right) \\
& =(-1)^{j_{1}-\mu_{1}}\left(\begin{array}{ccc}
j_{1} & j_{2} & j_{3} \\
-\mu_{1} & m_{2} & m_{3}
\end{array}\right) .
\end{aligned}
$$

Thus, to raise a quantum number we put the $1 \mathrm{jm}$-symbol on the right (and sum over the common index). Graphically, this raising is represented by an arrow reversal, since

$$
\left(\begin{array}{ccc}
\mu_{1} & j_{2} & j_{3} \\
j_{1} & m_{2} & m_{3}
\end{array}\right)=\sum_{m_{1}}+>_{3}+\frac{1}{>}>+<\mu_{1}
$$

as implied by equations (70) and (71).

To lower a magnetic quantum number, we write ( $c f$. equation (65))

$$
|j m\rangle=\sum_{\mu}|\vartheta(j \mu)\rangle\langle\vartheta(j \mu) \mid j m\rangle=\sum_{\mu}\left(\begin{array}{c}
j \\
m \mu
\end{array}\right)|\vartheta(j \mu)\rangle,
$$

which reads graphically

$$
D \longrightarrow>^{j m}=\sum_{\mu} D \stackrel{j \mu}{<} \ll \overbrace{}^{j \mu}>^{j m} .
$$

If we use this relationship in the first equation (83) and recall the definition of the mixed $3 \mathrm{jm}$-symbol, given in equation (88), we get

$$
\begin{aligned}
\left(\begin{array}{ccc}
j_{1} & j_{2} & j_{3} \\
m_{1} & m_{2} & m_{3}
\end{array}\right) & =\sum_{\mu_{1}}\left(\begin{array}{c}
j_{1} \\
m_{1} \mu_{1}
\end{array}\right)\left(\begin{array}{ccc}
\mu_{1} & j_{2} & j_{3} \\
j_{1} & m_{2} & m_{3}
\end{array}\right) \\
& =(-1)^{j_{1}+m_{1}}\left(\begin{array}{ccc}
-m_{1} & j_{2} & j_{3} \\
j_{1} & m_{2} & m_{3}
\end{array}\right) .
\end{aligned}
$$

Thus, to lower a magnetic quantum number we put the $1 \mathrm{jm}$-symbol on the left. Of course, raised and lowered $3 j m$-symbols are closely related. When we substitute $-m_{1}=\mu_{1}$ in the last equation and use that $j_{1}+m_{1}$ is an integer number, we immediately recover equation (89).

Clearly we can raise or lower the magnetic quantum numbers independently in each column. Correspondingly, this implies an independent arrow reversal for each of the corresponding lines of the $3 \mathrm{jm}$-diagram. This operation implies certain symmetry properties which follow from the (anti)unitarity of $\vartheta$ and the invariance of $\left\langle\left(j_{1} j_{2} j_{3}\right) 00\right|$ under $\vartheta$, which yield

$$
\left\langle\left(j_{1} j_{2} j_{3}\right) 00 \mid j_{1} m_{1} ; j_{2} m_{2} ; j_{3} m_{3}\right\rangle=\left\langle\left(j_{1} j_{2} j_{3}\right) 00 \mid \vartheta\left(j_{1} m_{1}\right) ; \vartheta\left(j_{2} m_{2}\right) ; \vartheta\left(j_{3} m_{3}\right)\right\rangle
$$


or

$$
\left(\begin{array}{ccc}
j_{1} & j_{2} & j_{3} \\
m_{1} & m_{2} & m_{3}
\end{array}\right)=\left(\begin{array}{ccc}
m_{1} & m_{2} & m_{3} \\
j_{1} & j_{2} & j_{3}
\end{array}\right) .
$$

In a very similar manner we obtain from equations (48) and (88)

$$
\left(\begin{array}{ccc}
m_{1} & j_{2} & j_{3} \\
j_{1} & m_{2} & m_{3}
\end{array}\right)=(-1)^{2 j_{1}}\left(\begin{array}{ccc}
j_{1} & m_{2} & m_{3} \\
m_{1} & j_{2} & j_{3}
\end{array}\right) .
$$

Graphically equation (91) gives the rule

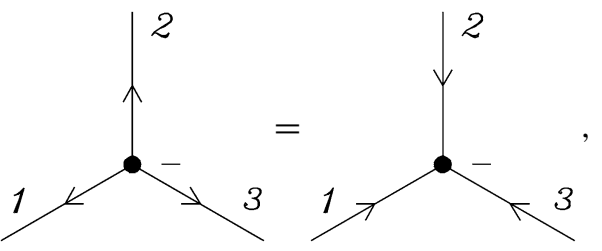

and equation (92):

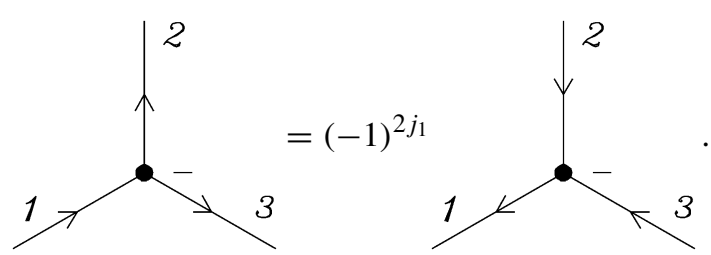

Note that in the latter rule the line with the unique variance determines the phase.

Finally, it is important to remark that also a mixed $3 j m$-symbol is invariant under an even permutation of its columns and obtains the phase factor $(-1)^{j_{1}+j_{2}+j_{3}}$ upon an odd permutation of its columns, which is due to the fact that the raising and lowering operations can be applied to any column independently.

\subsection{Transition from CG coefficients to $3 j \mathrm{j}$-symbols}

Almost all physical problems involving coupling of angular momenta find their most natural formulation in terms of CG coefficients. On the other hand, the $3 j m$-symbols are easier to manipulate and lead to simpler graphical rules in view of their symmetry. We thus find it best to formulate the initial problem in terms of CG diagrams and transform them subsequently to $3 \mathrm{jm}$-diagrams, using the rules which will be given shortly. We then construct the final resulting diagrams, simplify and evaluate them using the rules which will be expounded in the remainder of this work.

It is, of course, possible to formulate all the rules in terms of CG diagrams (see, e.g., Ref. [19]) or to employ a unified CG-3 jm representation [28]. In any case, however, either more complicated rules result or the generality must be sacrificed.

The transition rules that we now introduce are found by inspection. We will demonstrate their application on several examples in order to convince ourselves that they are correct.

RULES (Transformation from CG to $3 j m$-coefficients).

1. Conserve the variance and labeling of all lines and replace double arrows by single arrows. 
2. Change the vertex sign.

3. Multiply by $[j]^{1 / 2} \equiv(2 j+1)^{1 / 2}$, where $j$ labels the unique line of the CG coefficient.

4. Multiply by $(-1)^{2 j}$ for each line $j$ marked by an outgoing double arrow (a time-inverted bra).

5. Multiply by $(-1)^{2 j}$, where $j$ is the first non-unique line in the CG diagram. The first non-unique line is defined as the first line which is met when going in the direction indicated by the node sign starting from a single arrow unique line and in the opposite direction for a double arrow unique line.

To remember more easily rule 5, we recall that, loosely speaking, the bra is the complex conjugate of the ket, so that the association of complex with contra may provide a useful mnemonics.

In Table 1 we give a number of illustrations of the transformation rules with both the graphical and the algebraic form in each case. We illustrate rule 5 by a curved arrow ending at the first non-unique line. The transition rules for individual CG coefficients can be also applied to resulting CG diagrams containing contracted lines. In such a case we first assign arrows to the lines, which often gives us certain freedom.

Consider, for example, the diagram of the triangular delta, equation (64). Two of the possible arrow assignments are

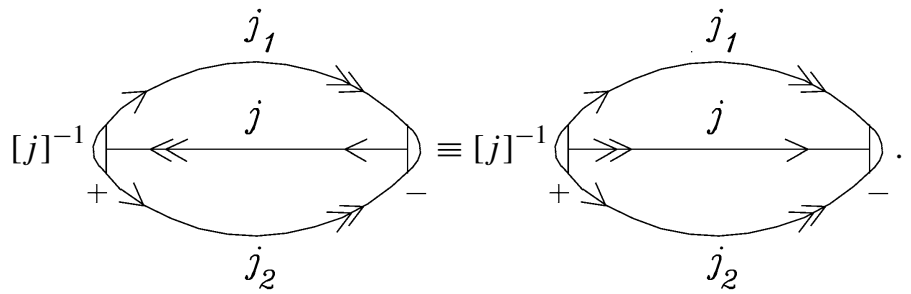

Application of the transition rules leads to, respectively,



$$
=\left\{j_{1}, j_{2}, j_{3}\right\} .
$$

Note that $(-1)^{2 j+2 j_{1}+2 j_{2}}=1$ and $(-1)^{2 j_{1}+2 j_{2}}=(-1)^{2 j}$, so that we have here an illustration of the rule of arrow reversal ( $c f$. (57)), which also holds for $3 j m$-diagrams.

Although it is irrelevant which arrow assignment we choose, as the above example illustrates, it must, of course, be done in a consistent way. The two non-unique lines that are attached to a given $\mathrm{CG}$ vertex must be both of the same type (i.e., either both bra or both ket lines). Further, a non-oriented contracted line must be composed of a bra and a ket, and an oriented line must consist either of two ket lines in the same direction or of two bra lines in opposite direction, as implied by the contraction Convention $\mathrm{C} 4$. In practical applications it is of course best to make the $\mathrm{CG} \rightarrow 3 \mathrm{jm}$ conversion first, before drawing the resulting diagrams, as already indicated above. 
Table 1. Examples of transformation from CG to $3 \mathrm{jm}$-diagrams

1.



$\left\langle j_{3} m_{3} \mid j_{1} m_{1} ; j_{2} m_{2}\right\rangle$

2.

$\left\langle\vartheta\left(j_{3} m_{3}\right) \mid j_{1} m_{1} ; j_{2} m_{2}\right\rangle$

$$
=\left[j_{3}\right]^{1 / 2}(-1)^{2 j_{2}}
$$

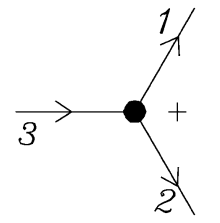

$=\left[j_{3}\right]^{1 / 2}(-1)^{2 j_{2}}$ $\left(\begin{array}{ccc}j_{1} & m_{3} & j_{2} \\ m_{1} & j_{3} & m_{2}\end{array}\right)$

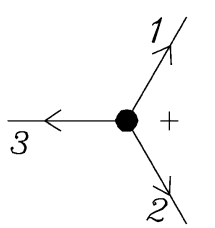

$\left(\begin{array}{ccc}j_{1} & j_{3} & j_{2} \\ m_{1} & m_{3} & m_{2}\end{array}\right)$

3.



$$
=\left[j_{3}\right]^{1 / 2}(-1)^{2 j_{1}}
$$

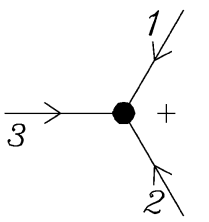

$\left\langle j_{1} m_{1} ; j_{2} m_{2}\right| \vartheta\left(j_{3} m_{3}\right)$

$=\left[j_{3}\right]^{1 / 2}(-1)^{2 j_{1}}$

$\left(\begin{array}{ccc}m_{1} & m_{3} & m_{2} \\ j_{1} & j_{3} & j_{2}\end{array}\right)$

4.


$\left\langle\vartheta\left(j_{2} m_{2}\right) ; j_{1} m_{1} \mid j_{3} m_{3}\right\rangle$ $=\left[j_{3}\right]^{1 / 2}(-1)^{2 j_{1}+2 j_{2}+2 j_{2}}$

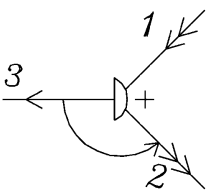

$\left(\begin{array}{ccc}j_{1} & j_{2} & j_{3} \\ m_{1} & m_{2} & m_{3}\end{array}\right)$

$=\left[j_{3}\right]^{1 / 2}(-1)^{4 j_{2}}$

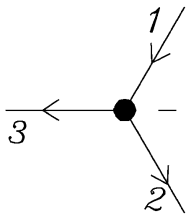

$\left(\begin{array}{ccc}m_{1} & j_{2} & j_{3} \\ j_{1} & m_{2} & m_{3}\end{array}\right)$

\section{BASIC RULES FOR ANGULAR MOMENTUM DIAGRAMS}

In the preceding section we have introduced the basic concepts of graphical techniques of spin algebras and formulated the rules for the conversion of CG diagrams into 
$3 j m$-diagrams. From now on we shall restrict our attention to the latter and will derive the rules which will enable us to reduce any angular momentum problem to the computation of canonical $3 n j$-symbols, $n=1,2, \ldots$, by expressing the kinematic part of a given angular momentum problem in terms of these symbols. The dynamic part, however, which is contained in the so-called reduced matrix elements, must be treated separately for each case.

In deriving the graphical rules we shall distinguish closed and open diagrams and will represent them as closed and open boxes,

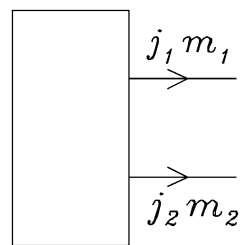

(a)

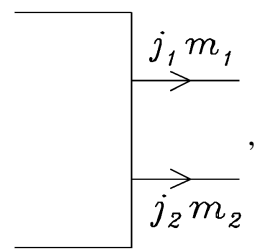

(b)

respectively. Thus, the diagram (94a) represents a graph with an arbitrary number of $3 j m$-vertices and with all lines contracted except those which are shown explicitly, namely the lines labeled $j_{1} m_{1}$ and $j_{2} m_{2}$. Referring to the contracted lines which connect two $3 \mathrm{jm}$-vertices as the internal lines and to the uncontracted ones as the free or external lines, we can also say that a closed diagram represented by a full box, as in (94a), contains only internal lines, and all the free lines must be shown explicitly. With an open box, diagram (94b), we then represent a graph which may possess additional free lines to those indicated explicitly. Since some of the rules hold only for closed diagrams, this distinction is important.

\subsection{Removal of a zero line}

We prove the following rules for the removal of a zero line, which hold provided that $j_{1}$ and $j_{2}$ are distinct lines.

RULE 1.

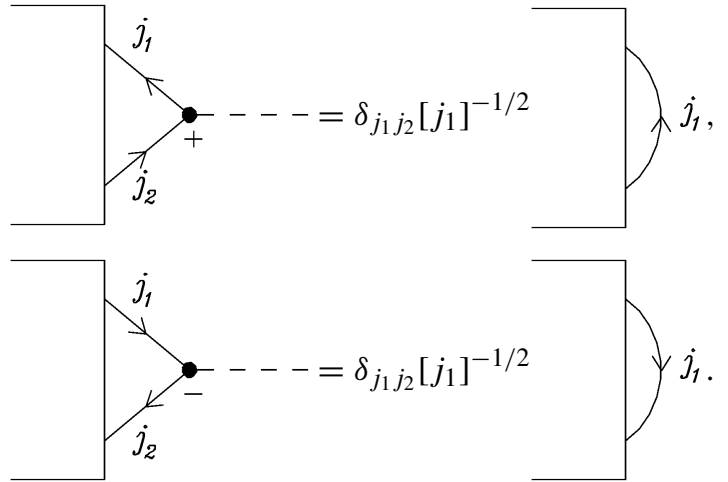

Thus, when the lines $j_{1}$ and $j_{2}$ run in the direction indicated by the vertex sign, the vertex with attached zero line can be replaced by the factor $\delta_{j_{1} j_{2}}\left[j_{1}\right]^{-1 / 2}$. 
PROOF. Assuming that $j_{1}$ and $j_{2}$ are distinct lines, we may reverse the direction of the $j_{2}$ line and write

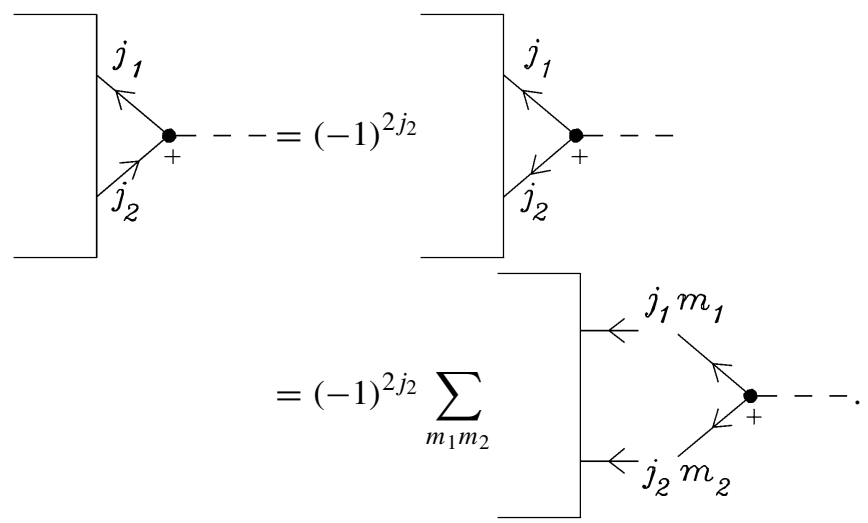

Converting to a CG vertex and using equation (74) we find

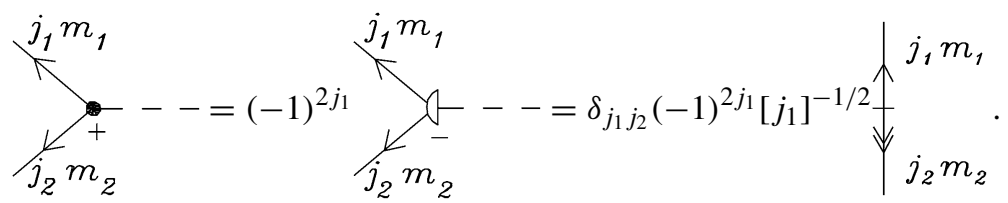

Using, further, the completeness relation, equation (71), we get the first rule, (95),

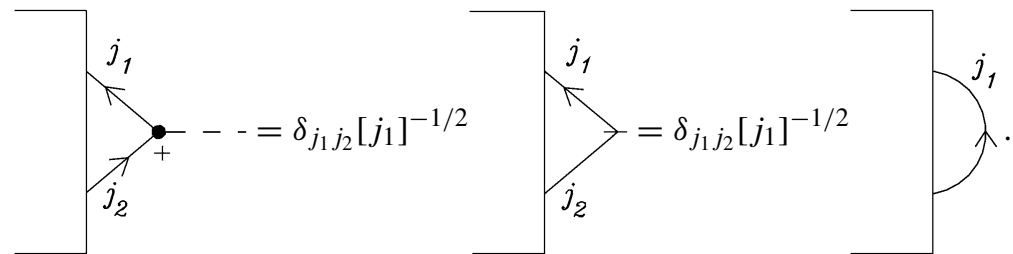

The second rule, (96), follows directly from the first when we note that

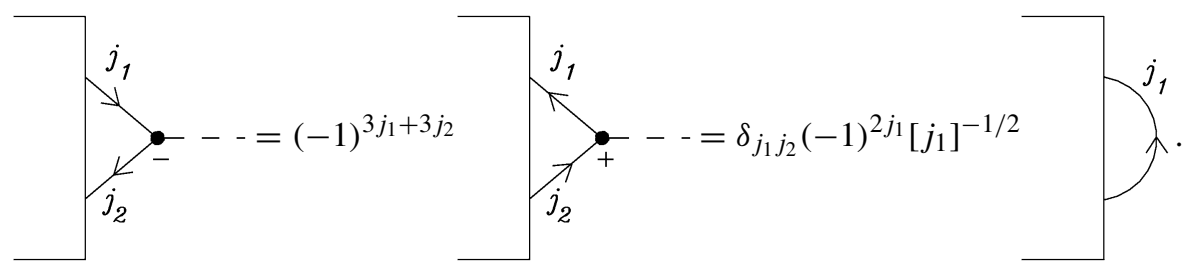

The use of the rule for arrow reversal then gives the desired result.

REMARK. It is of interest to consider the case-which we explicitly excluded-that $j_{1}$ and $j_{2}$ label the same line and for which the proof above does not apply. Since by equation (72)

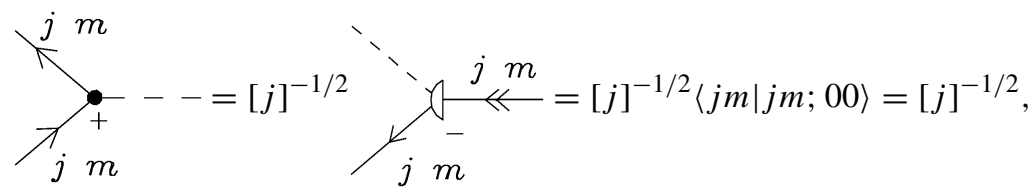


we find that

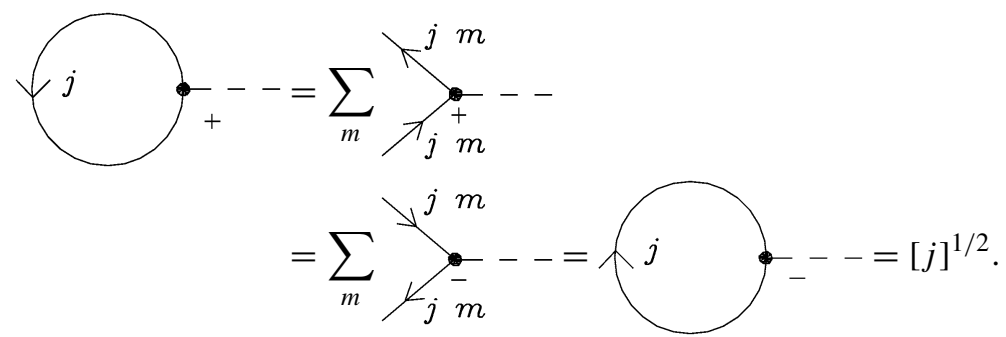

Recalling that the non-oriented loop represents $[j] \equiv 2 j+1$, equation (55), we get the rule

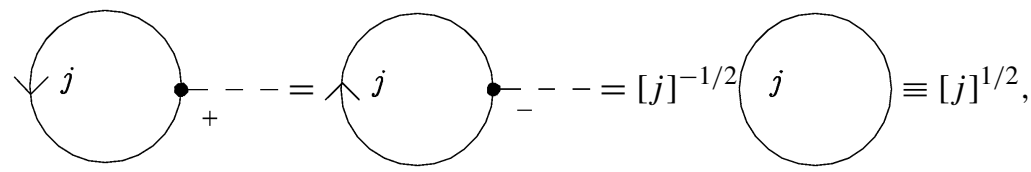

which differs from the general rules (95) and (96) in that the resulting line is non-oriented. It is essential that the loop is non-oriented, because otherwise the rule of arrow reversal would predict that the two oriented loops differ by a factor $(-1)^{2 j}$.

\subsection{Factorization rules}

A $3 j m$-symbol, being an inner product by equation (83), is an $\mathrm{SU}(2)$ invariant. On the other hand, it transforms as a triple Kronecker product of irreps, equation (83). A similar observation leads to the well-known Wigner-Eckart theorem, which states that the matrix element of an irreducible tensor operator between eigenstates of $J^{2}$ can be factored into an invariant quantity (the so-called reduced matrix element) and a quantity that reflects the transformation properties of the matrix element. The latter quantity is a mixed $3 \mathrm{jm}$-symbol (or a CG coefficient).

We shall first show that the transformation properties of a diagram are solely determined by its free lines. Following the proof of the Wigner-Eckart theorem we then obtain a factorization of the diagram into an invariant quantity and a quantity that carries the transformation properties of the diagram.

Consider a diagram with $n 3 \mathrm{jm}$-vertices (the proof works equally well for CG graphs). Transforming all the states with $U \in \mathrm{SU}(2)$, the diagram, on the one hand, remains invariant and, on the other hand, transforms as a $3 n$-fold Kronecker product of $D$ matrices. However, the $D$ matrices that correspond to internal lines cancel one another in view of their unitarity. Indeed, by definition, a contracted line consists of a standard and a contrastandard quantity, say a ket and a time-reversed ket, so that

$$
\begin{aligned}
U \sum_{m}|j m ; \vartheta(j m)\rangle & =\sum_{m, \mu, \mu^{\prime}} D^{(j)}(U)_{\mu m} D^{(j)}(U)_{\mu^{\prime} m}^{*}\left|j \mu ; \vartheta\left(j \mu^{\prime}\right)\right\rangle \\
& =\sum_{\mu, \mu^{\prime}} \delta_{\mu \mu^{\prime}}\left|j \mu ; \vartheta\left(j \mu^{\prime}\right)\right\rangle=\sum_{\mu}|j \mu ; \vartheta(j \mu)\rangle .
\end{aligned}
$$

(The invariance of contractions of other types is proved in a similar manner, $c f$. also equation (35).) Thus, the transformation properties of a diagram are defined solely and uniquely 
by its free lines. We next state and prove the following very useful rule for the closing of a diagram with two external lines.

RULE 2.

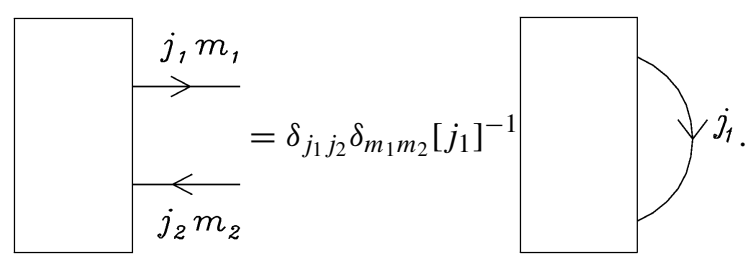

PROOF. We denote the diagram on the left-hand side by $G\left(\begin{array}{cc}j_{1} & m_{2} \\ m_{1} & j_{2}\end{array}\right)$ and apply $U \in \mathrm{SU}(2)$ obtaining

$$
\begin{aligned}
U G\left(\begin{array}{cc}
j_{1} & m_{2} \\
m_{1} & j_{2}
\end{array}\right) & =G\left(\begin{array}{cc}
j_{1} & m_{2} \\
m_{1} & j_{2}
\end{array}\right) \\
& =\sum_{\mu_{1}, \mu_{2}} D^{\left(j_{1}\right)}(U)_{\mu_{1} m_{1}} D^{\left(j_{2}\right)}(U)_{\mu_{2} m_{2}}^{*} G\left(\begin{array}{cc}
j_{1} & \mu_{2} \\
\mu_{1} & j_{2}
\end{array}\right) .
\end{aligned}
$$

Since all unitary groups are compact, their "volume" is well defined, and designating the volume of $\mathrm{SU}(2)$ by $V$, we find using the great orthonormality relations and integrating over the whole group, that

$$
\begin{aligned}
V G\left(\begin{array}{cc}
j_{1} & m_{2} \\
m_{1} & j_{2}
\end{array}\right) & =\sum_{\mu_{1}, \mu_{2}} G\left(\begin{array}{cc}
j_{1} & \mu_{2} \\
\mu_{1} & j_{2}
\end{array}\right) \int D^{\left(j_{1}\right)}(U)_{\mu_{1} m_{1}} D^{\left(j_{2}\right)}\left(U^{-1}\right)_{m_{2} \mu_{2}} d V \\
& =\sum_{\mu_{1}, \mu_{2}} G\left(\begin{array}{cc}
j_{1} & \mu_{2} \\
\mu_{1} & j_{2}
\end{array}\right) \delta_{j_{1} j_{2}} \delta_{\mu_{1} \mu_{2}} \delta_{m_{1} m_{2}} V\left[j_{1}\right]^{-1} \\
& =\delta_{j_{1} j_{2}} \delta_{m_{1} m_{2}} V\left[j_{1}\right]^{-1} \sum_{\mu_{1}} G\left(\begin{array}{cc}
j_{1} & \mu_{1} \\
\mu_{1} & j_{1}
\end{array}\right),
\end{aligned}
$$

so that

$$
G\left(\begin{array}{cc}
j_{1} & m_{2} \\
m_{1} & j_{2}
\end{array}\right)=\delta_{j_{1} j_{2}} \delta_{m_{1} m_{2}}\left[j_{1}\right]^{-1} \sum_{\mu_{1}} G\left(\begin{array}{cc}
j_{1} & \mu_{1} \\
\mu_{1} & j_{1}
\end{array}\right)
$$

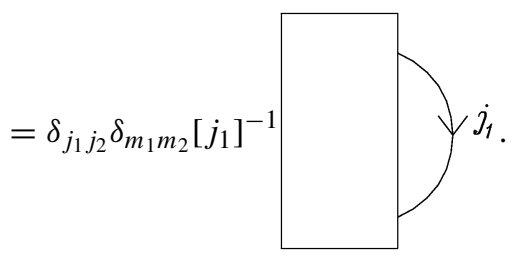

In a similar way we can prove the following rule:

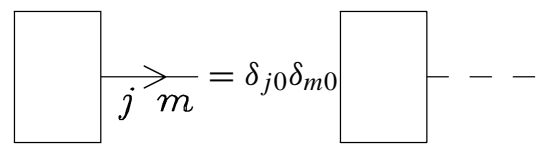

which states that the only SU(2) invariant quantity transforming according to the irrep $(j)$ of $\mathrm{SU}(2)$ is one transforming according to the identity irrep $j=0$. We must emphasize 
that the above two rules hold irrespective of whether the diagram consists of CG vertices, $3 \mathrm{jm}$-vertices or a mixture of both. In the case of two free lines, the proof simply rests on the fact that one line is standard and the other one contrastandard.

To derive similar rules for diagrams with more than two free lines we simply precouple the lines, thus reducing their number. This is most easily done by exploiting the identity



which follows immediately from the corresponding orthogonality relation for CG coefficients, equation (63).

RULE 3. A diagram can be factorized over three lines by the use of
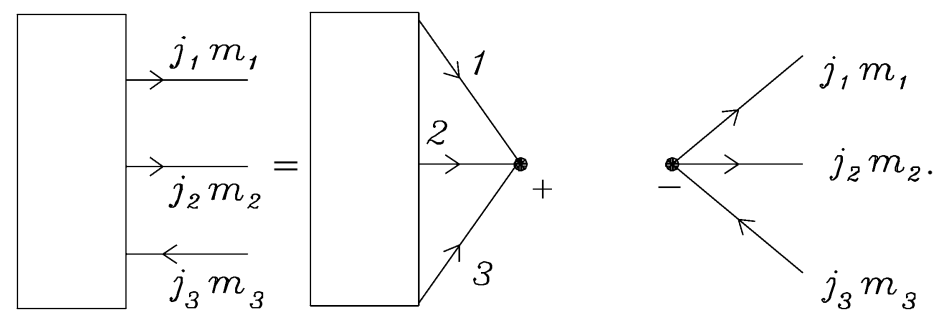

PROOF. Applying the identity (100) and the separation rule (98), we obtain

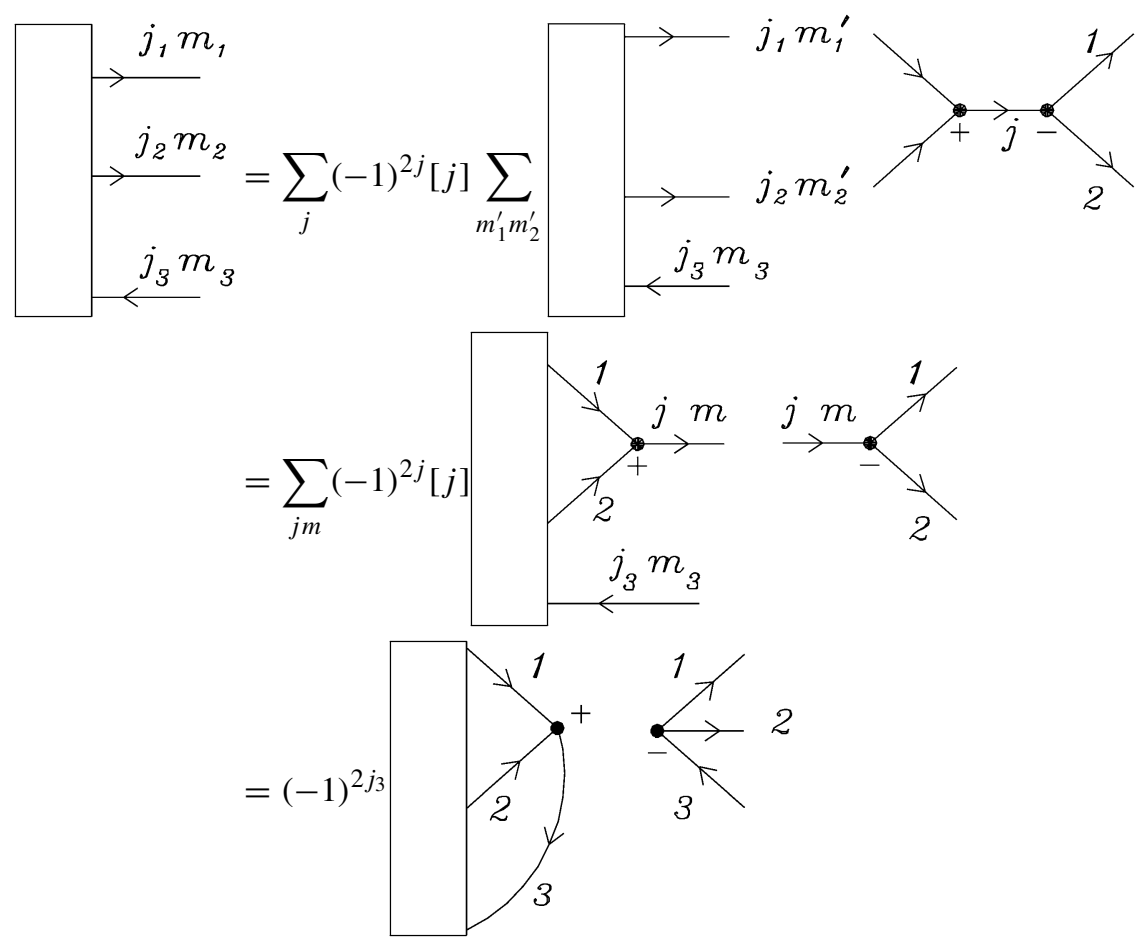




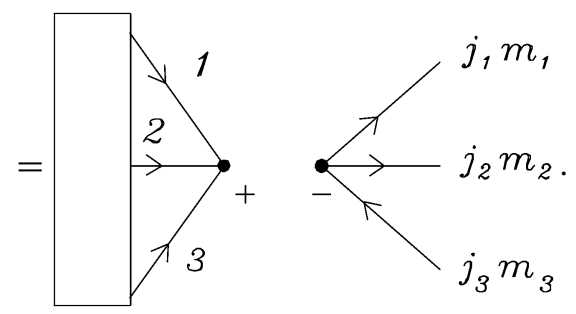

Similar rules hold for other possible orientations of external lines (see the example below).

Note that we have achieved the same factorization as in the Wigner-Eckart theorem. The closed diagram is invariant under $\mathrm{SU}(2)$, and the mixed $3 \mathrm{jm}$-symbol has the transformation properties of the original diagram, characterized by two standard (outgoing) lines and one contrastandard (ingoing) line. The positions of the plus and minus vertex signs in the final result may be interchanged, since $(-1)^{2\left(j_{1}+j_{2}+j_{3}\right)}=1$.

In the case of more than three external lines we can simply factor out the corresponding generalized CG coefficient or its $3 j m$-equivalent. Thus, in the case of four external lines, we find the following rule.

RULE 4. A diagram can be separated over four lines by the use of the following rule,

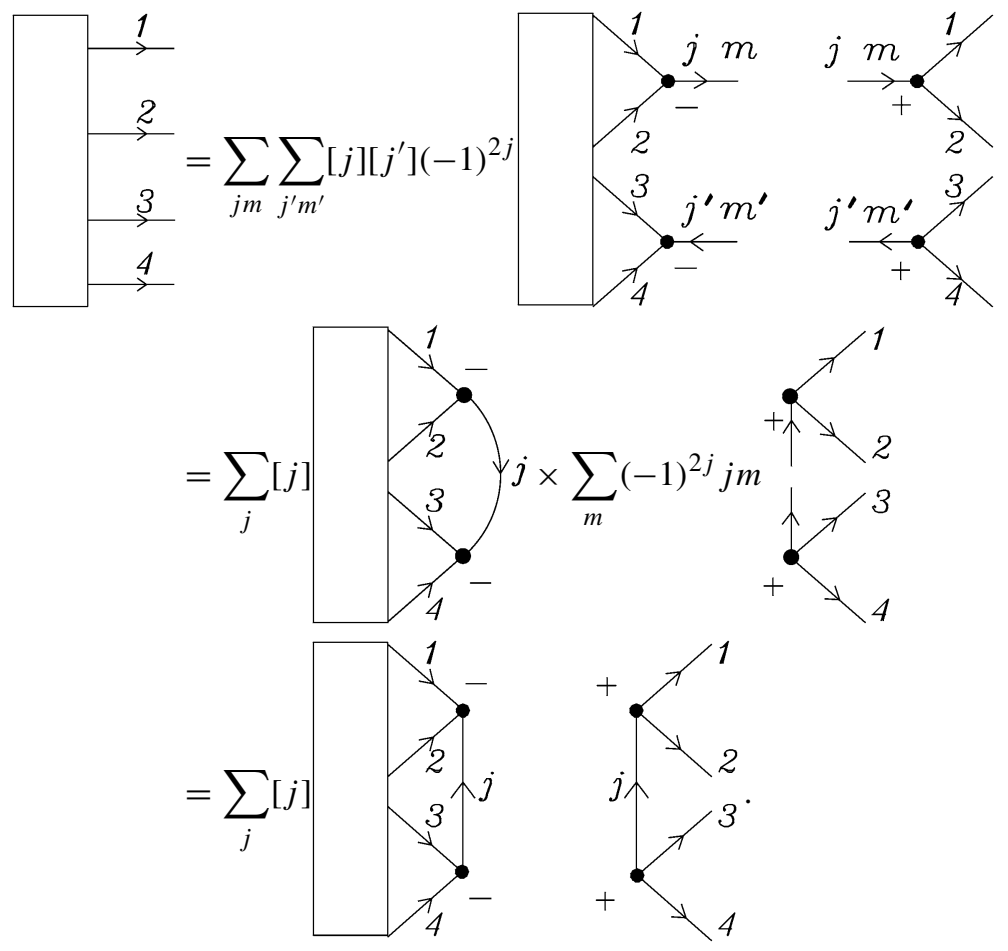

Note that both $j$ lines could also be oriented downward and that the vertex signs are arbitrary as long as they are opposite for vertices associated with identically labeled lines. 
It should now be clear how to handle the cases with more than four free lines. However, since $n$ free lines, $n>3$, give rise to $n-3$ summations over the intermediate quantum numbers, the factorization rule is seldom used for more than four free lines.

When we meet open diagrams whose free lines have variances different from those used in the above given factorization rules, we proceed in the same way as we did in the raising and lowering of the magnetic quantum numbers in $3 \mathrm{jm}$-symbols, Section 3.5. Namely, we use either one of the following rules:

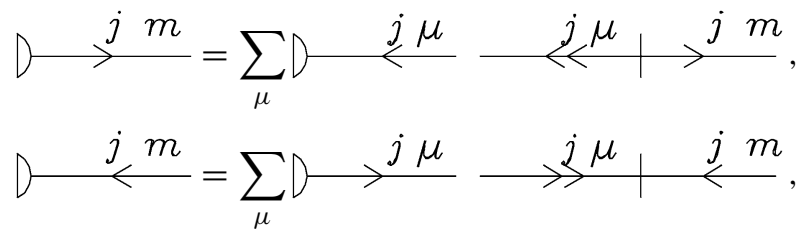

which converts the free line to the required variance, and then apply the factorization rule to the line labeled by the summation index $\mu$. For example,

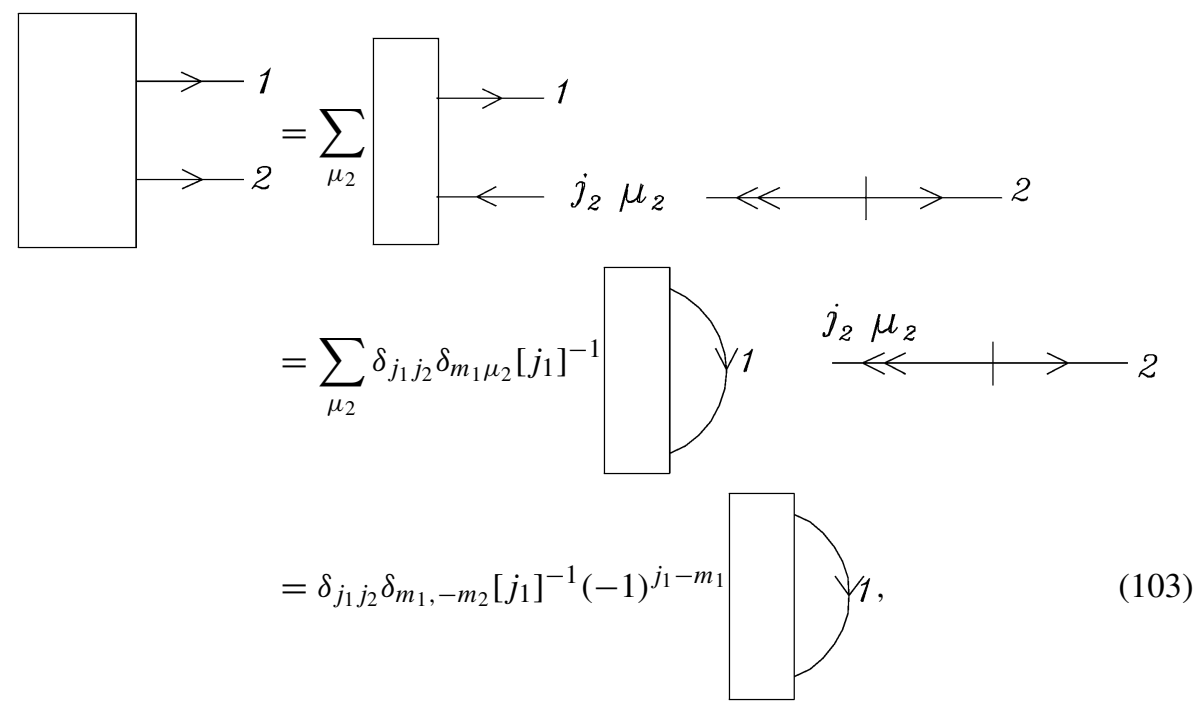

where we used $\left\langle\vartheta\left(j_{2} \mu_{2}\right) \mid j_{2} m_{2}\right\rangle=(-1)^{j_{2}+m_{2}} \delta_{-\mu_{2}, m_{2}}$ (equation (67)) for the $1 \mathrm{jm}$-symbol. Similarly,

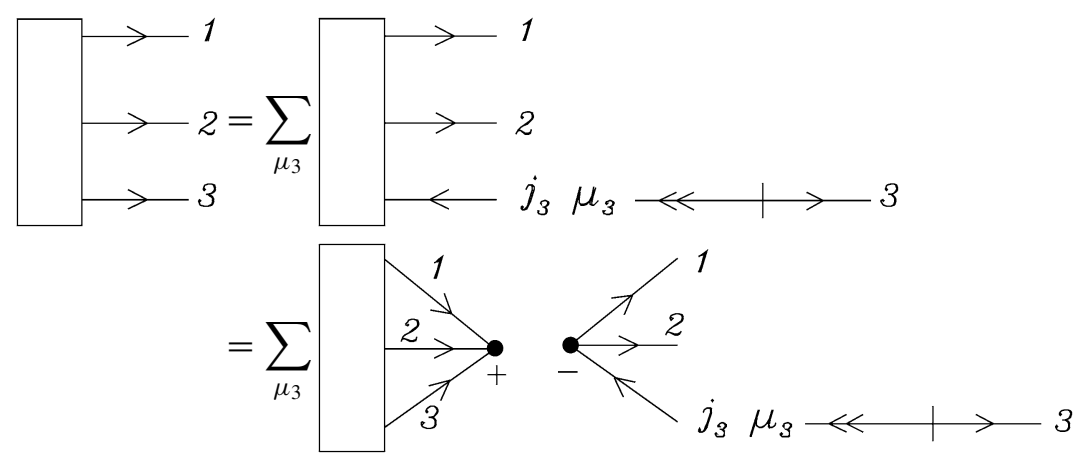




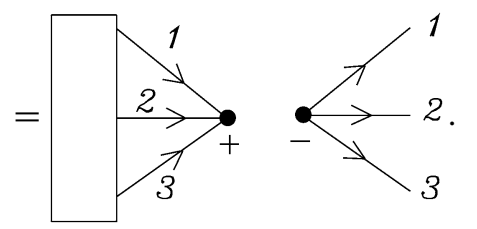

\subsection{Separation rules}

The factorization rules imply immediately the following separation rules.

1. Separation over one line:

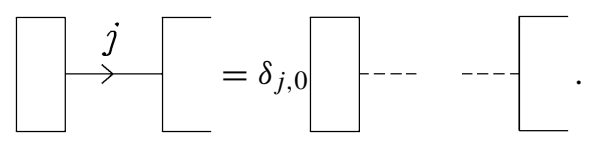

The zero angular momentum lines can then be eliminated by the use of rule (95), (96), or (97).

2. Separation over two lines:

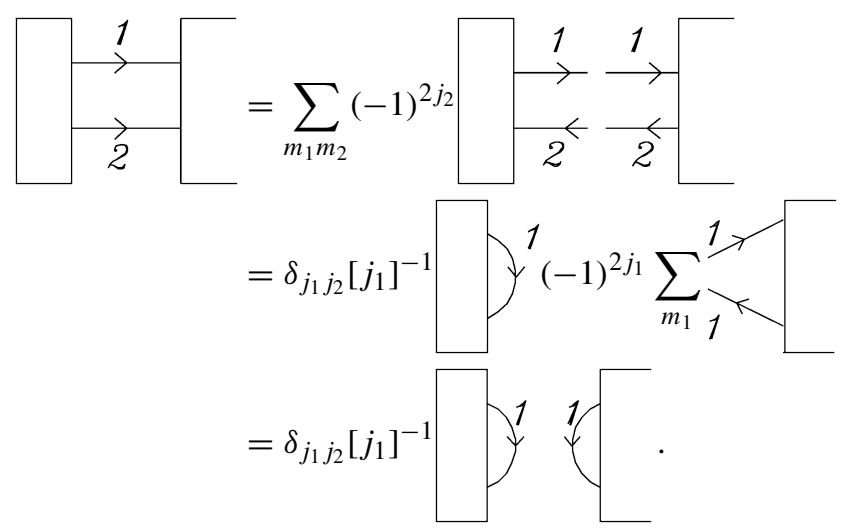

Clearly, both resulting $j_{1}$ lines can also be oriented in the upward direction. Similarly, if the $j_{1}$ and $j_{2}$ lines run in opposite directions in the connected diagram at the left-hand side, then so do the resulting lines in the separated diagram.

3. Separation over three lines: Using the factorization rule equation (104), we can prove that
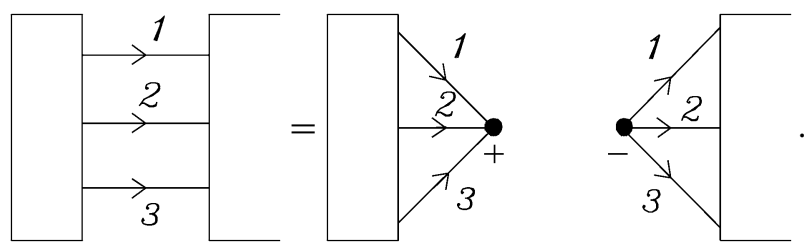

We could also formulate the rules for separation over more than three lines. However, such rules may be difficult to apply, particularly within complicated diagrams, and it is generally advisable to proceed by substituting one or more intermediate summation lines. We have already applied this technique of pairwise precoupling in the factorization of 
diagrams with three and four external lines. After a sufficient number of summation lines have been introduced, the diagram may be factored using one of the separation rules given above.

The substitution of a summation line is accomplished by the following rule, which is a direct consequence of equation (100),

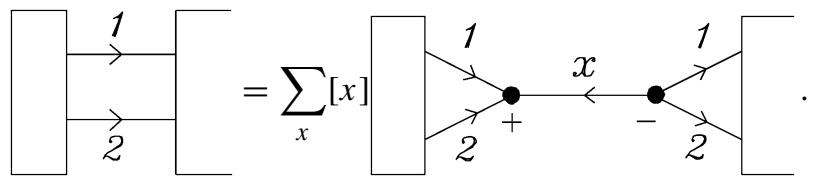

This rule is easy to remember: we first orient the lines to be coupled so that they run in the same direction. We insert a summation line that runs in the opposite direction. The vertex signs are chosen in such a way that the order of the three angular momenta $j_{1}, j_{2}$, and $x$ is the same for both vertices. (Note that the diagram is invariant with respect to the interchange of vertex signs.) An analogous rule holds also for the case of crossing lines.

4. Separation over four lines: We separate over four lines, as an example precoupling $j_{2}$ and $j_{3}$,

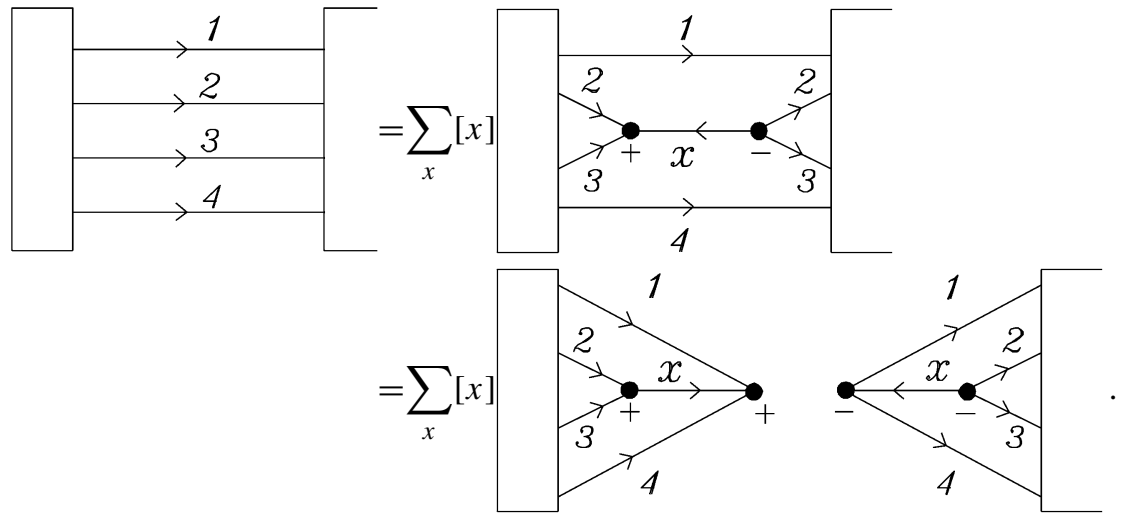

(109)

To end the discussion of the separation rules we point out that the factorization rules represent a special case of the separation rules when one of the diagrams is an empty diagram. It thus suffices to remember the separation rules only.

We will end this section by giving four examples in which the separation rules are illustrated.

EXAMPLE 1. As an illustration of the two-line separation rule (106), we consider the removal of a loop, i.e., a diagram of the type

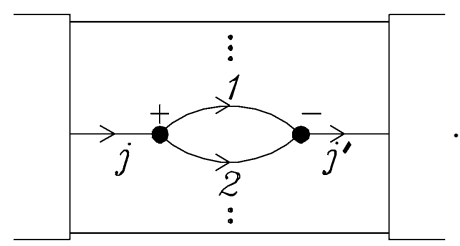


Pulling down the loop sufficiently far and observing that the loop is a closed diagram, we can apply (106) for the lines $j$ and $j^{\prime}$, obtaining

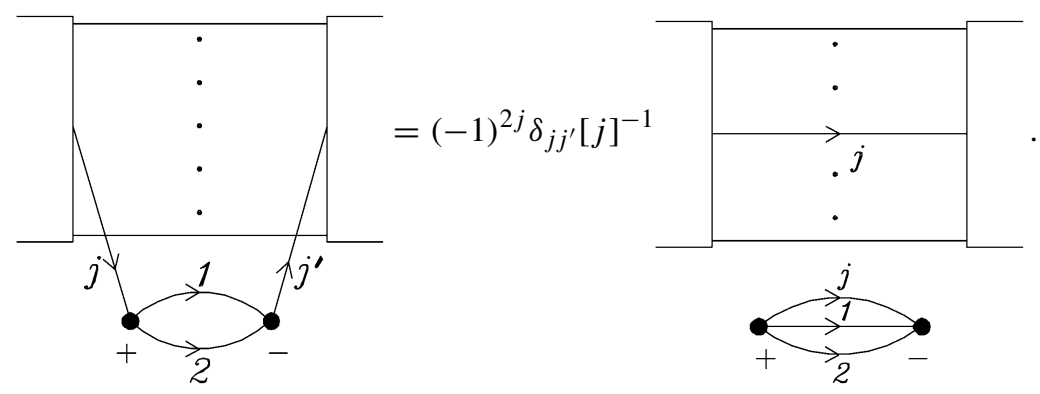

We have already met both the CG and the $3 j m$-forms of the "oyster" diagram (equations (64) and (93), respectively). The $3 j m$-form is equal to the triangular delta $\left\{j, j_{1}, j_{2}\right\}$ (which is unity when the triangular conditions hold).

EXAMPLE 2. To illustrate a separation over three and four lines, we consider the example given in Fig. 1 where we precoupled $j_{5}$ and $j_{6}$. Clearly, we could also first apply the precoupling rule (108) to the lines $j_{1}$ and $j_{2}$ in Fig. 1 and then factor over the three lines.

EXAMPLE 3. As another example we consider the diagram $G$ in Fig. 2. There are two obvious ways of separation over the four lines, vertically: $j_{1}^{\prime}, j_{2}^{\prime}, j_{3}^{\prime}, j_{4}^{\prime}$ (represented by $1^{\prime}$, $2^{\prime}, 3^{\prime}, 4^{\prime}$ ), and horizontally: $j_{1}, j_{2}, j_{5}, j_{6}$. In the first case we get the diagram in Fig. 3 . The second possibility leads clearly to the same final result. The resulting four diagrams are irreducible and cannot be factored into smaller closed diagrams. In Section 5 we shall return to these closed diagrams.
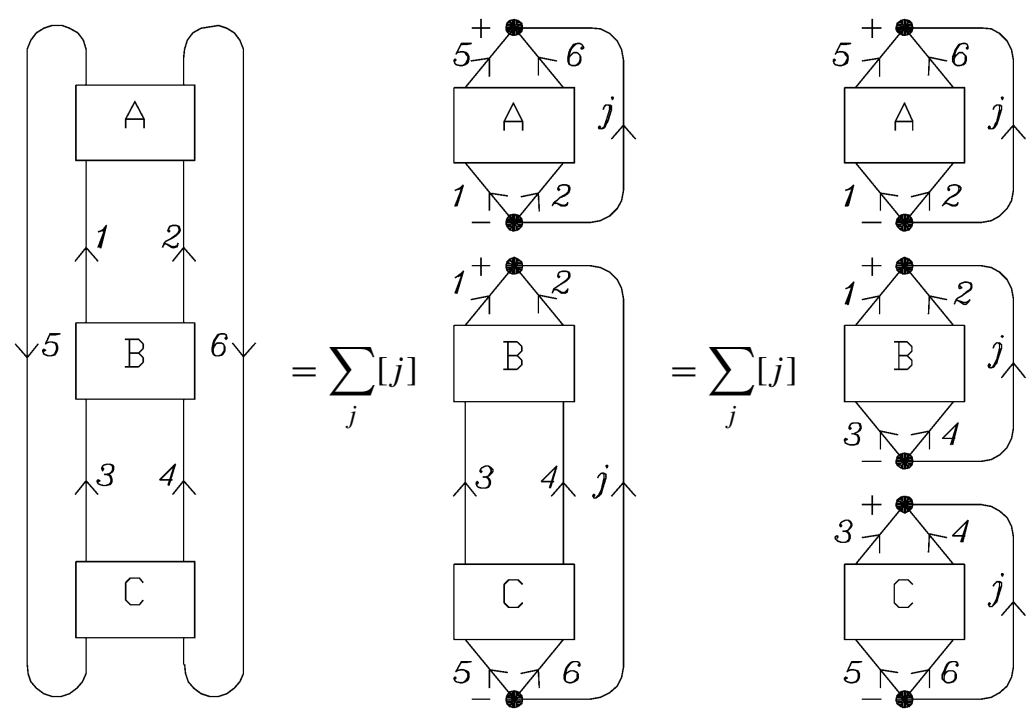

Fig. 1. An illustration of a separation over four lines (Example 2). 


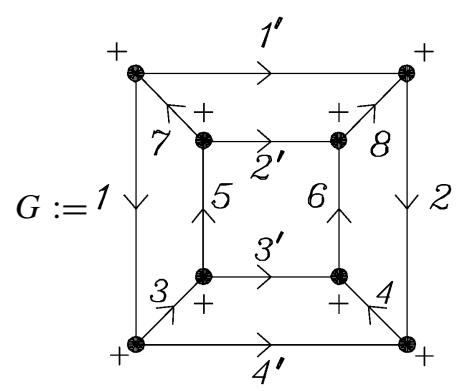

Fig. 2. The initial diagram of Example 3.

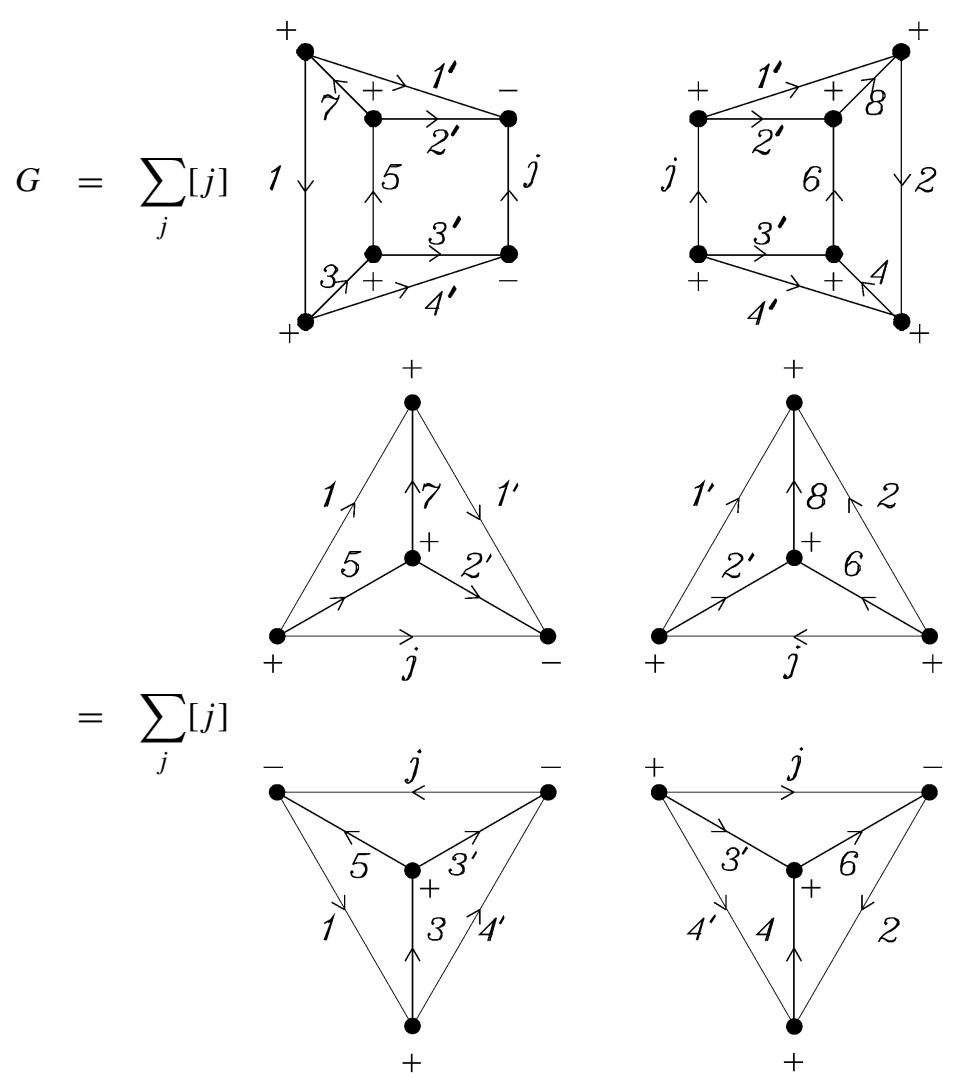

Fig. 3. The diagram of Fig. 2 (Example 3) after separation over four lines.

EXAMPLE 4. There is also a less obvious way to factor $G$ of Fig. 2. To clarify this possibility, we first convert $G$ into a form (Fig. 4) that is topologically the same as the diagram in Fig. 2. By using equation (100) we now precouple $j_{1}$ and $j_{2}$ and draw the summation line $j$ vertically in the center of the diagram. Then we separate three times over three lines. The diagrams on the right-hand side of Fig. 4 are also irreducible. In fact, they can be easily redrawn into the "tetrahedral" shape used in the first factorization of $G$, see Fig. 3. 


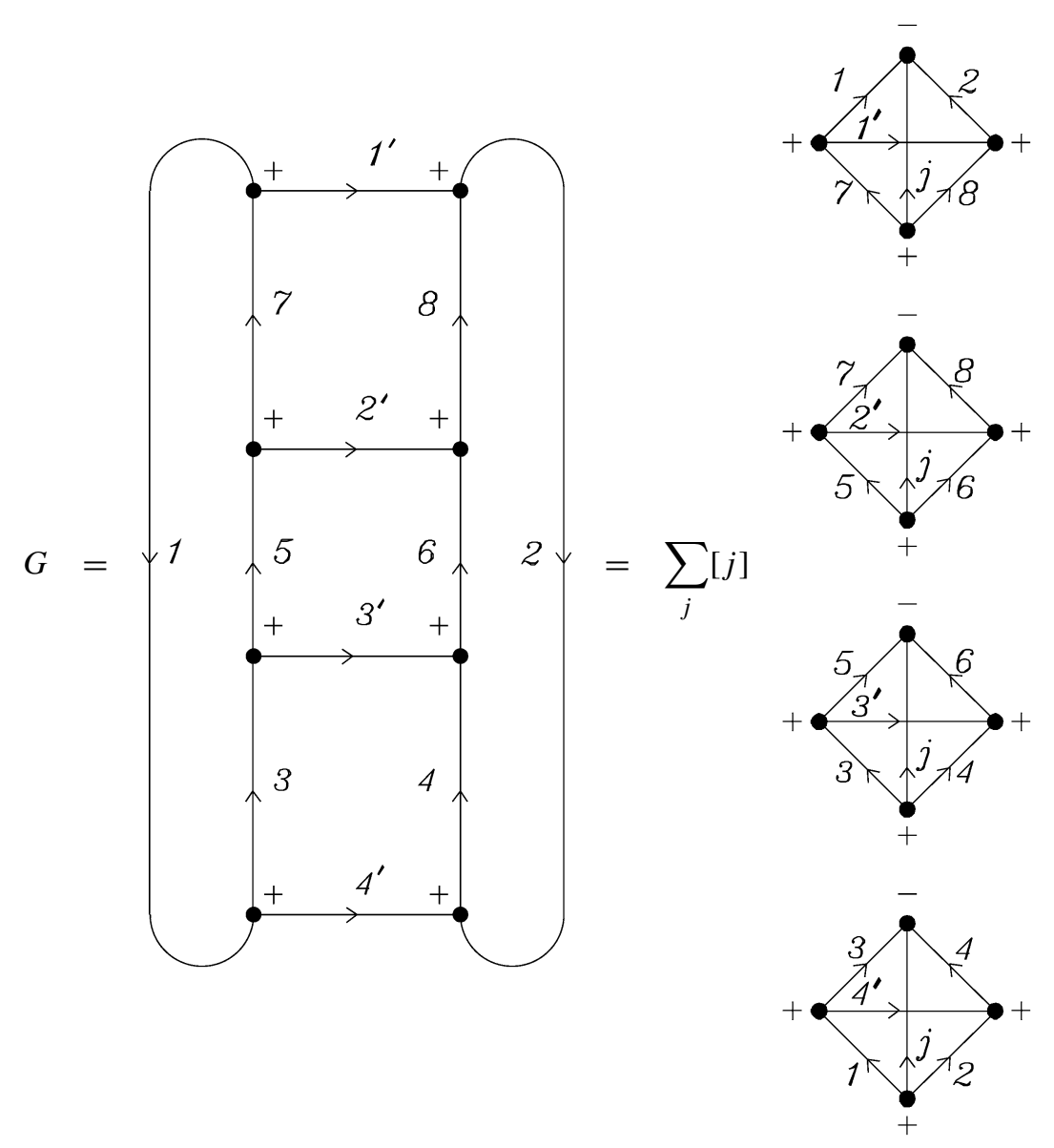

Fig. 4. Separation of the diagram of Example 4. Note that $G$ is the same diagram as in Fig. 2.

Note that the different diagrams in the previous two examples contain different angular momenta and, consequently, are related in a non-trivial way (through a so-called sum rule).

\section{IRREDUCIBLE CLOSED DIAGRAMS}

The main objective of graphical methods for the study of an angular momentum problem is to reduce it to the form of a product of standard quantities that are easy to handle and to evaluate. We have seen how to factor a general open diagram into a product of a closed diagram and a diagram representing a generalized CG coefficient. The latter diagram is clearly the simplest possible diagram containing a given number of external lines. We thus turn our attention to the closed diagrams.

A closed diagram is reducible if it can be separated into a product of closed diagrams, otherwise it is irreducible. The factorization of a closed diagram into irreducible components provides clearly the utmost simplification of the angular momentum problem. 
Suppose we have an irreducible diagram consisting of $k>0$ vertices. Since three lines are attached to every vertex and every line connects two vertices, the total number of lines equals $3 k / 2$. Hence, the number of vertices in an irreducible diagram must be even, say $k=2 n, n=1,2, \ldots$. We thus find the general result that an irreducible diagram consists of $3 n$ lines and $2 n$ vertices. Since each line is labeled by a quantum number $j$, we speak of 3nj-symbols.

\section{1. $1 j$ - and $3 j$-symbols}

We have already encountered a closed (and irreducible) diagram containing one line and no vertices $(k=0)$, namely

$$
\sum_{m}\langle j m \mid j m\rangle=[j] \equiv 2 j+1=\square^{j} .
$$

It is appropriate to refer to this non-oriented loop as a $1 j$-symbol.

The $3 j$-symbol (or triangular delta) was already introduced in Section 2.5 in CG form (see Section 3.5 for the $3 j m$-form),

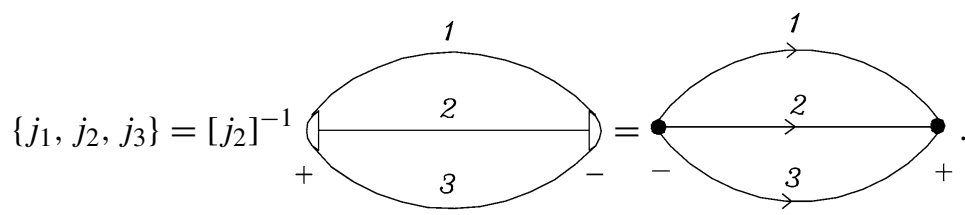

Its value is zero or unity depending on whether the three angular momenta satisfy the triangular condition. The $3 j m$-representation of $\left\{j_{1}, j_{2}, j_{3}\right\}$ shows clearly its symmetry: A transposition of two of the three angular momenta gives the phase $(-1)^{2 J}$, where $J:=j_{1}+j_{2}+j_{3}$. Since $J$ is an integer, it follows that the $3 j$-symbol is invariant under permutations.

\subsection{Recoupling and the $6 j$ - and $9 j$-symbols}

Because the $6 j-, 9 j-$, and higher symbols appear naturally in the problem of recoupling of three, four (and more) angular momenta, we first discuss this problem before turning to the study of their properties.

We have seen in Section 3.4 that we can couple three or more angular momenta by different coupling schemes. This does not imply that eigenstates of $J^{2}$, obtained by different coupling schemes, are linearly independent. Indeed, states obtained in one coupling scheme can be expressed as linear combinations of states obtained by another coupling scheme. This is called recoupling. Recoupling is a basis transformation in tensor product space $V_{j_{1}} \otimes V_{j_{2}} \otimes \cdots \otimes V_{j_{n}}$, where $V_{j_{k}}$ is a $\left(2 j_{k}+1\right)$-dimensional carrier space of an irrep of SU(2). Specification of a coupling scheme (genealogy) leads to a basis for this space and another coupling scheme gives another basis; recoupling transforms the one basis into the other.

To be more specific we first consider the case of three angular momenta. In Section 3.4 we have considered two coupling schemes, namely $\left(\left(j_{1} j_{2}\right) j_{12} j_{3}\right)$ and $\left(j_{1}\left(j_{2} j_{3}\right) j_{23}\right)$. We 
relate the states obtained by these different couplings by

$$
\begin{aligned}
& \left|\left(\left(j_{1} j_{2}\right) j_{12} j_{3}\right) j m\right\rangle \\
& \quad=\sum_{j_{23}, j^{\prime}, m^{\prime}}\left|\left(j_{1}\left(j_{2} j_{3}\right) j_{23}\right) j^{\prime} m^{\prime}\right\rangle\left\langle\left(j_{1}\left(j_{2} j_{3}\right) j_{23}\right) j^{\prime} m^{\prime} \mid\left(\left(j_{1} j_{2}\right) j_{12} j_{3}\right) j m\right\rangle .
\end{aligned}
$$

The expansion coefficients arising in this basis transformation are recoupling coefficients. The recoupling coefficient obviously vanishes unless $j=j^{\prime}$ and $m=m^{\prime}$, and we shall see shortly that it is independent of $m$. Both facts follow immediately from the diagrammatic rules.

In order to obtain a graphical representation of the recoupling coefficients, we substitute the resolution of the identity, expressed in terms of the uncoupled basis, obtaining the generalized CG coefficients of Section 3.4 (see (77) and (78)). Thus,

$$
\begin{aligned}
R:= & \left\langle\left(j_{1}\left(j_{2} j_{3}\right) j_{23}\right) j^{\prime} m^{\prime} \mid\left(\left(j_{1} j_{2}\right) j_{12} j_{3}\right) j m\right\rangle \\
= & \sum_{m_{1} m_{2} m_{3}}\left\langle\left(j_{1}\left(j_{2} j_{3}\right) j_{23}\right) j^{\prime} m^{\prime} \mid j_{1} m_{1} ; j_{2} m_{2} ; j_{3} m_{3}\right\rangle \\
& \times\left\langle j_{1} m_{1} ; j_{2} m_{2} ; j_{3} m_{3} \mid\left(\left(j_{1} j_{2}\right) j_{12} j_{3}\right) j m\right\rangle
\end{aligned}
$$
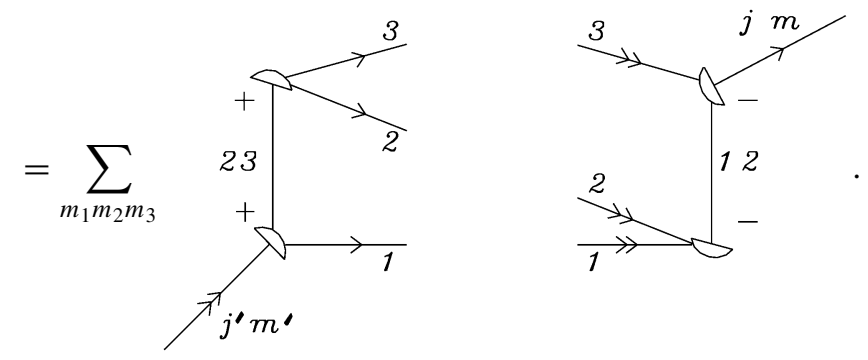

Transition to $3 j m$-diagrams and introduction of the notation

$$
\left[j_{1}, j_{2}, \ldots, j_{k}\right]:=\prod_{i=1}^{k}\left[j_{i}\right] \equiv \prod_{i=1}^{k}\left(2 j_{i}+1\right), \quad k>0,
$$

gives

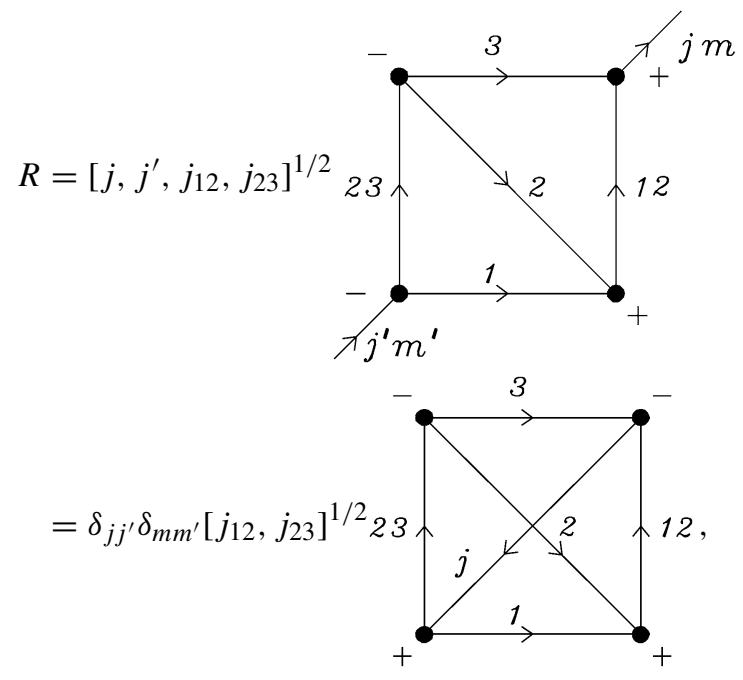


where in the last step we have applied the rule (98). The closed diagram that we have obtained cannot be further factored into closed diagrams in a non-trivial way: it is irreducible. (Note that by the rule (101) an oyster type diagram can be separated off from each corner; this, however, is a trivial factorization.)

The diagram of the recoupling coefficient is proportional to Wigner's $6 j$-symbol, which is defined graphically as

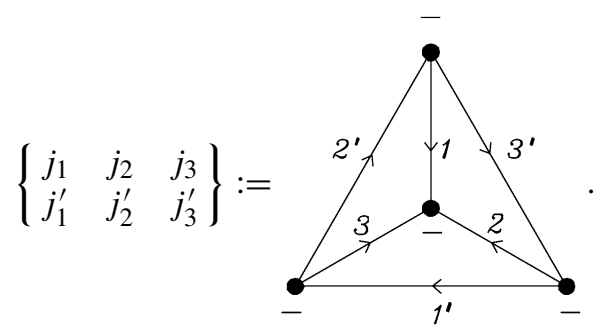

This definition may be remembered by noting that $j_{1}, j_{2}$, and $j_{3}$ appear on the central vertex in the order indicated by the vertex sign. The angular momentum $j_{k}^{\prime}$ appears on the edge opposite $j_{k}, k=1,2,3$. Hence, the direction of $j_{1}^{\prime}, j_{2}^{\prime}, j_{3}^{\prime}$ is also dictated by the central vertex sign. All vertices have the same sign. Obviously, the graph can be drawn in several different forms, which imply its symmetry properties. We shall address this problem in the next section.

Bringing the diagram in equation (110) to this standard form, we finally get

$$
\begin{aligned}
R & \equiv\left\langle\left(j_{1}\left(j_{2} j_{3}\right) j_{23}\right) j^{\prime} m^{\prime} \mid\left(\left(j_{1} j_{2}\right) j_{12} j_{3}\right) j m\right\rangle \\
& =\delta_{j j^{\prime}} \delta_{m m^{\prime}}(-1)^{j_{1}+j_{2}+j_{3}+j_{12}}\left[j_{12}, j_{23}\right]^{1 / 2} \\
& =\delta_{j j^{\prime}} \delta_{m m^{\prime}}(-1)^{j_{1}+j_{2}+j_{3}+j}\left[j_{12}, j_{23}\right]^{1 / 2}\left\{\begin{array}{ccc}
j_{1} & j & j_{12} \\
j_{3} & j & j_{23}
\end{array}\right\} .
\end{aligned}
$$

We next consider the recoupling of four angular momenta, where several coupling schemes are again possible. We can couple sequentially, one angular momentum after another, or we can also couple pairwise, in which case we have the possible coupling schemes $\left(\left(j_{1} j_{2}\right)\left(j_{3} j_{4}\right)\right),\left(\left(j_{1} j_{3}\right)\left(j_{2} j_{4}\right)\right)$, and $\left(\left(j_{1} j_{4}\right)\left(j_{2} j_{3}\right)\right)$. All recoupling problems can be easily treated by the graphical techniques. As an example we consider the recoupling

$$
\begin{aligned}
& \left|\left(\left(j_{1} j_{2}\right) j_{12}\left(j_{3} j_{4}\right) j_{34}\right) j m\right\rangle \\
& =\sum_{j_{13}, j_{24}}\left|\left(\left(j_{1} j_{3}\right) j_{13}\left(j_{2} j_{4}\right) j_{24}\right) j m\right\rangle \\
& \quad \times\left[\sum_{m_{1} m_{2} m_{3} m_{4}}\left\langle\left(\left(j_{1} j_{3}\right) j_{13}\left(j_{2} j_{4}\right) j_{24}\right) j m \mid j_{1} m_{1} ; j_{2} m_{2} ; j_{3} m_{3} ; j_{4} m_{4}\right\rangle\right. \\
& \left.\times\left\langle j_{1} m_{1} ; j_{2} m_{2} ; j_{3} m_{3} ; j_{4} m_{4} \mid\left(\left(j_{1} j_{2}\right) j_{12}\left(j_{3} j_{4}\right) j_{34}\right) j m\right\rangle\right] .
\end{aligned}
$$


The expression in square brackets is the recoupling coefficient expressed in terms of generalized CG coefficients and has the following graphical representation
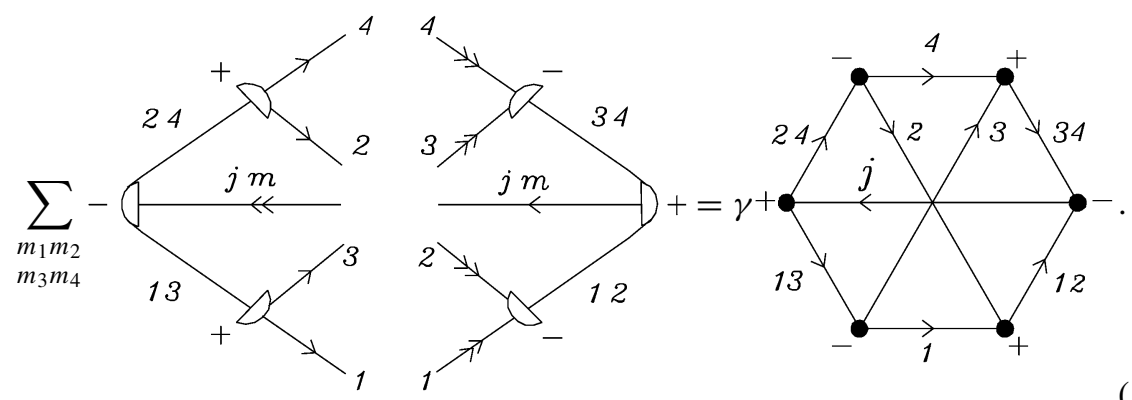

The factor $\gamma$ which arises from the transition to the $3 \mathrm{jm}$-representation and from application of equation (98) to the free lines with $j m$ labels is easily found to be

$$
\begin{aligned}
\gamma & =\left[j_{13}, j, j_{24}, j_{34}, j, j_{12}\right]^{1 / 2}[j]^{-1}(-1)^{2 j_{3}+2 j_{24}+2 j_{4}+2 j_{3}+2 j_{12}+2 j_{1}} \\
& =\left[j_{13}, j_{24}, j_{34}, j_{12}\right]^{1 / 2} .
\end{aligned}
$$

The hexagonal diagram in equation (114) is proportional to Wigner's $9 j$-symbol, which we define as

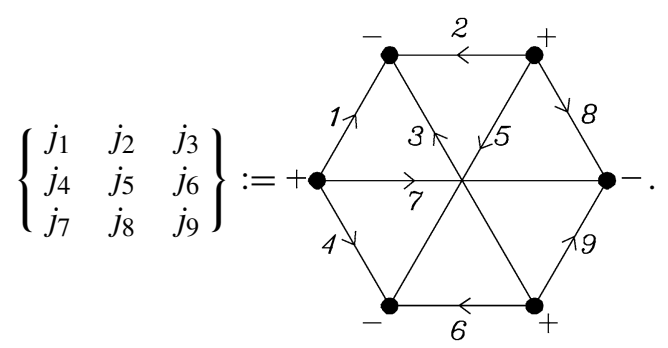

To remember this definition we note that:

- The vertex signs on the perimeter of the hexagon alternate.

- The nodes with minus signs define the rows of the $9 j$-symbol and those with plus signs define its columns.

- The order within the rows and columns is also defined by the node signs.

- The arrows always point from the positive to negative vertices.

In the next section we shall show that there are different, though equivalent, ways of representing the $9 j$-symbol, which reflect its various symmetries.

In order to bring the $9 j$-symbol of equation (114), defining the recoupling coefficient, to the standard form, equation (116), we first flip all the vertex signs. This does not change the phase since

$$
(-1)^{2\left(j_{1}+j_{3}+j_{13}\right)+2\left(j_{2}+j_{4}+j_{24}\right)+2\left(j_{12}+j_{34}+j\right)}=1 .
$$

Reversing, further, the orientation on intermediately coupled lines again keeps the phase unchanged, since

$$
(-1)^{2\left(j_{13}+j_{24}+j_{34}+j_{12}\right)}=(-1)^{4 j}=1,
$$


so that we finally get

$$
\begin{array}{r}
\left\langle\left(j_{1} j_{3}\right) j_{13}\left(j_{2} j_{4}\right) j_{24} j m \mid\left(j_{1} j_{2}\right) j_{12}\left(j_{3} j_{4}\right) j_{34} j m\right\rangle \\
=\left[j_{13}, j_{24}, j_{12}, j_{34}\right]^{1 / 2}\left\{\begin{array}{ccc}
j_{1} & j_{2} & j_{12} \\
j_{3} & j_{4} & j_{34} \\
j_{13} & j_{24} & j
\end{array}\right\} .
\end{array}
$$

\subsection{Properties of the $6 j$-symbol}

We see from the defining equation (111) that the $6 j$-symbol can be written as the following complete contraction of four $3 \mathrm{jm}$-symbols

$$
\begin{aligned}
&\left\{\begin{array}{ccc}
j_{1} & j_{2} & j_{3} \\
j_{1}^{\prime} & j_{2}^{\prime} & j_{3}^{\prime}
\end{array}\right\}=\sum_{\substack{m_{1}, m_{2}, m_{3} \\
m_{1}^{\prime}, m_{2}^{\prime}, m_{3}^{\prime}}}\left(\begin{array}{ccc}
m_{1} & m_{2} & m_{3} \\
j_{1} & j_{2} & j_{3}
\end{array}\right)\left(\begin{array}{ccc}
j_{1} & m_{2}^{\prime} & j_{3}^{\prime} \\
m_{1} & j_{2}^{\prime} & m_{3}^{\prime}
\end{array}\right) \\
& \times\left(\begin{array}{ccc}
j_{1}^{\prime} & j_{2} & m_{3}^{\prime} \\
m_{1}^{\prime} & m_{2} & j_{3}^{\prime}
\end{array}\right)\left(\begin{array}{ccc}
m_{1}^{\prime} & j_{2}^{\prime} & j_{3} \\
j_{1}^{\prime} & m_{2}^{\prime} & m_{3}
\end{array}\right) .
\end{aligned}
$$

Since the following four relations must hold,

$$
0=m_{1}+m_{2}+m_{3}=m_{1}-m_{2}^{\prime}+m_{3}^{\prime}=m_{1}^{\prime}+m_{2}-m_{3}^{\prime}=-m_{1}^{\prime}+m_{2}^{\prime}+m_{3},
$$

the sum is, in fact, only a double sum. Each $3 j m$-symbol, being proportional to a CG coefficient, is given by a single sum $(c f .(51))$, so that a $6 j$-symbol represents a six-fold summation. However, through a series of ingenious substitutions, Racah [29] was able to reduce the 6-fold summation to the following formula (for details, see [13], Chapter 3, Appendix A)

$$
\begin{aligned}
& \left\{\begin{array}{rrr}
j_{1} & j_{2} & j_{3} \\
j_{1}^{\prime} & j_{2}^{\prime} & j_{3}^{\prime}
\end{array}\right\} \\
& =\Delta\left(j_{1} j_{2} j_{3}\right) \Delta\left(j_{1} j_{2}^{\prime} j_{3}^{\prime}\right) \Delta\left(j_{1}^{\prime} j_{2} j_{3}^{\prime}\right) \Delta\left(j_{1}^{\prime} j_{2}^{\prime} j_{3}\right) \\
& \quad \times \sum_{t}(-1)^{t}(t+1) ! \\
& \quad \times\left[\left(t-j_{1}-j_{2}-j_{3}\right) !\left(t-j_{1}-j_{2}^{\prime}-j_{3}^{\prime}\right) !\left(t-j_{1}^{\prime}-j_{2}-j_{3}^{\prime}\right) !\right. \\
& \quad \times\left(t-j_{1}^{\prime}-j_{2}^{\prime}-j_{3}\right) !\left(j_{1}+j_{2}+j_{1}^{\prime}+j_{2}^{\prime}-t\right) ! \\
& \left.\quad \times\left(j_{1}+j_{3}+j_{1}^{\prime}+j_{3}^{\prime}-t\right) !\left(j_{2}+j_{3}+j_{2}^{\prime}+j_{3}^{\prime}-t\right) !\right]^{-1},
\end{aligned}
$$

where

$$
\Delta(a b c):=\left[\frac{(a+b-c) !(a-b+c) !(-a+b+c) !}{(a+b+c+1) !}\right]^{1 / 2} .
$$

The sum over $t$ is restricted by the requirement that the factorials occurring in (119) are non-negative.

The $6 j$-symbol is invariant under any permutation of its vertices

Hence, its symmetry group is the permutation group $S_{4}$, which is of order 4 ! (all permutations of 4 distinct objects). By considering the diagram of the $6 j$-symbol one easily proves 
this symmetry. Indeed,

$$
\begin{aligned}
\left\{\begin{array}{lll}
j_{1} & j_{2} & j_{3} \\
j_{1}^{\prime} & j_{2}^{\prime} & j_{3}^{\prime}
\end{array}\right\} & =\left\{\begin{array}{lll}
j_{a} & j_{b} & j_{c} \\
j_{a}^{\prime} & j_{b}^{\prime} & j_{c}^{\prime}
\end{array}\right\}=\left\{\begin{array}{lll}
j_{a} & j_{b}^{\prime} & j_{c}^{\prime} \\
j_{a}^{\prime} & j_{b} & j_{c}
\end{array}\right\} \\
& =\left\{\begin{array}{lll}
j_{a}^{\prime} & j_{b} & j_{c}^{\prime} \\
j_{a} & j_{b}^{\prime} & j_{c}
\end{array}\right\}=\left\{\begin{array}{lll}
j_{a}^{\prime} & j_{b}^{\prime} & j_{c} \\
j_{a} & j_{b} & j_{c}^{\prime}
\end{array}\right\},
\end{aligned}
$$

where $(a b c)$ is any permutation of $\left(\begin{array}{ll}1 & 2\end{array}\right)$. For instance, the invariance with respect to the transposition of the first and last column follows by interchanging the vertices $\mathrm{C}$ and $\mathrm{D}$ in the following diagram:

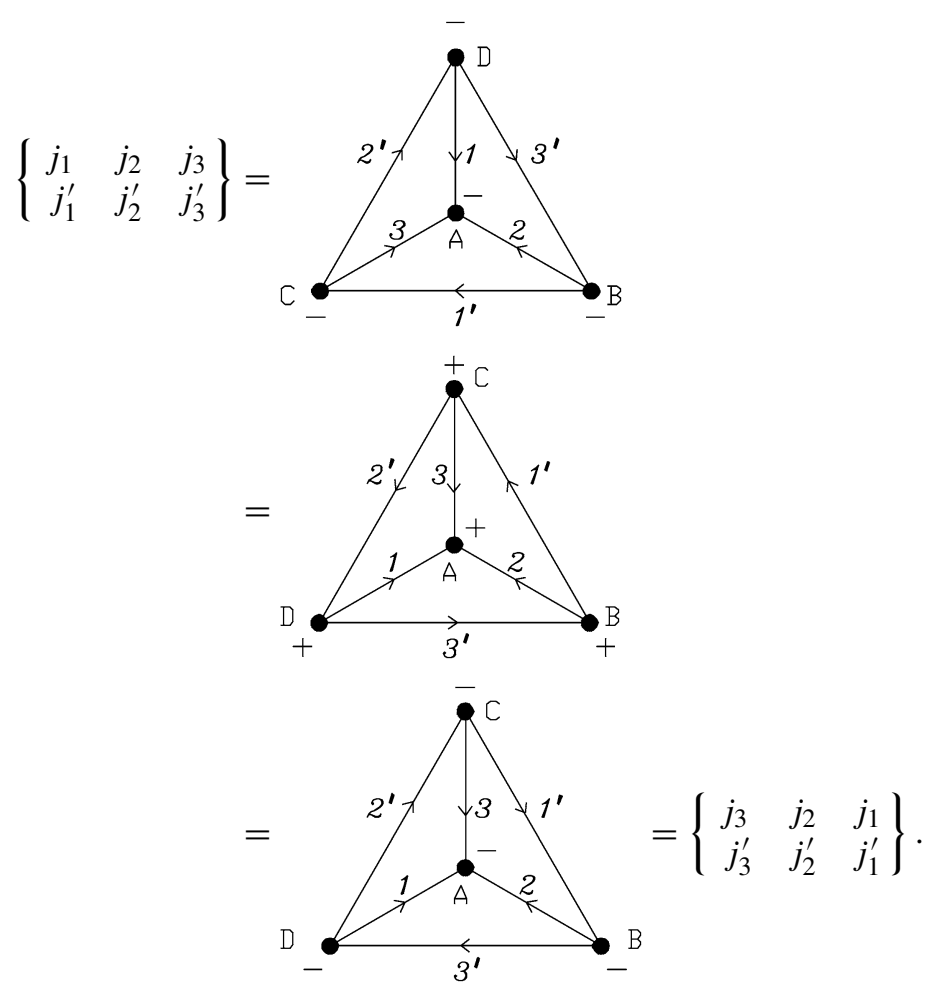

It is easily checked that the phase arising from the conversion of the second diagram into the third one equals unity. The second diagram represents, in fact, another canonical form which is easy to remember: all vertices carry the same sign, the lines on the perimeter are oriented in the direction indicated by the signs, and all lines attached to the central vertex are ingoing (or outgoing).

The $6 j$-symbol is invariant with respect to the interchange of the rows in any two of its columns

Consider for instance the cyclic permutation (A B C) applied to the first diagram of equa- 
tion (121), which gives

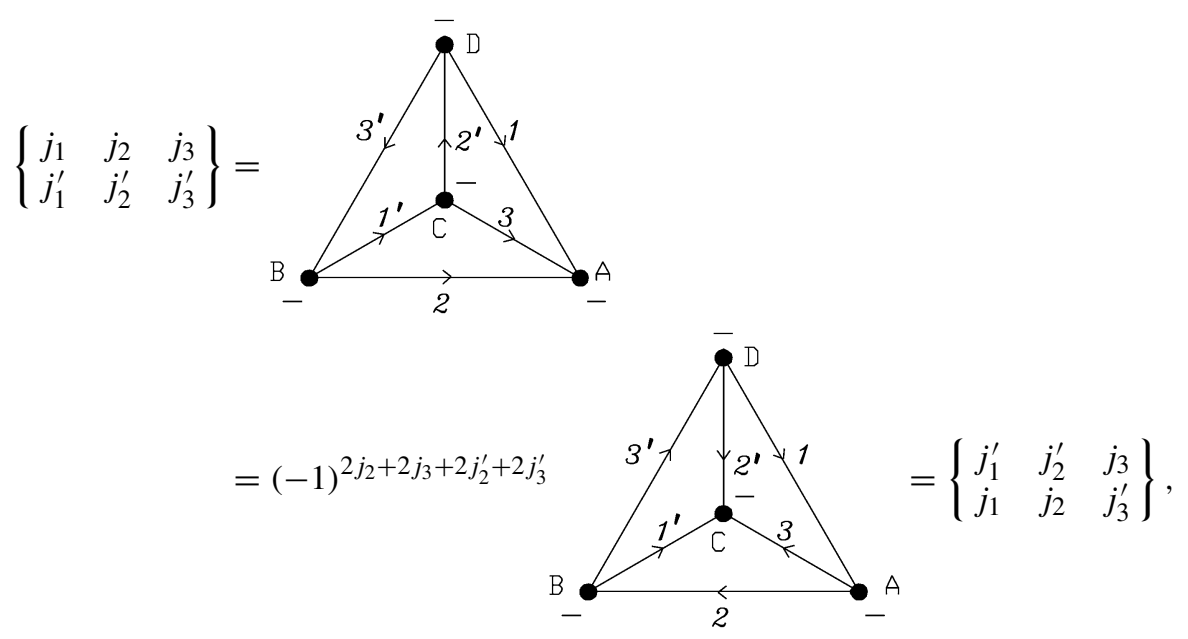

where we used $(-1)^{2 j_{2}+2 j_{3}}=(-1)^{2 j_{2}^{\prime}+2 j_{3}^{\prime}}=(-1)^{2 j_{1}}$.

Besides the 24 symmetry operations, equation (120), there exists an additional symmetry, first found by Regge [30] by inspection of the Racah formula for the $6 j$-symbol. We forego a discussion of the Regge symmetry and only mention it here for completeness.

The graphical method is very powerful for deriving sum rules. For example,

$$
\sum_{j_{1}}\left[j_{1}\right](-1)^{2 j_{1}}\left\{\begin{array}{lll}
j_{1} & j_{2} & j_{3} \\
j_{1}^{\prime} & j_{2} & j_{3}
\end{array}\right\}=\left\{j_{1}^{\prime}, j_{2}, j_{3}\right\} .
$$

To prove this we use the completeness relation, equation (100), and obtain

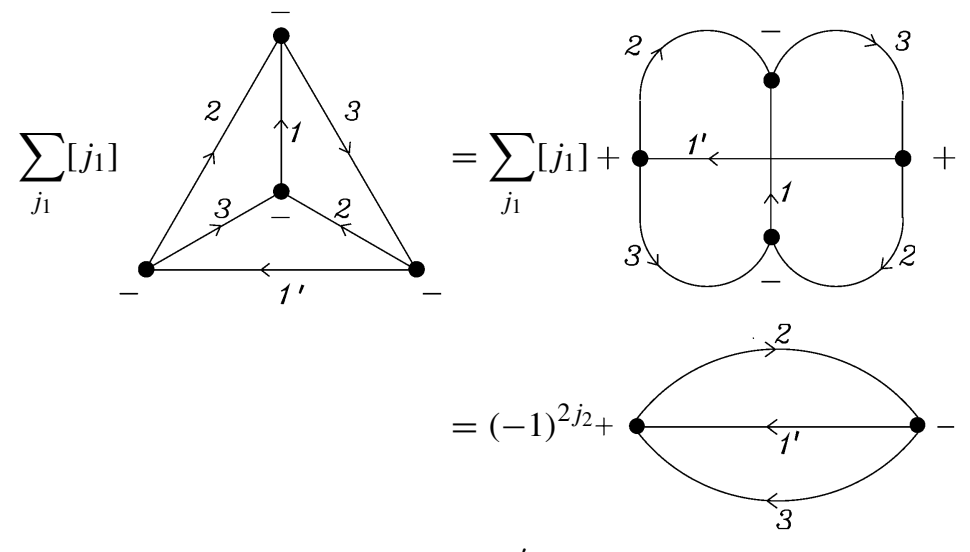

$=\left\{j_{1}^{\prime}, j_{2}, j_{3}\right\}$.

Similarly, setting $j_{2}^{\prime}=j_{3}$ and $j_{3}^{\prime}=j_{2}$, we get

$$
\sum_{j_{1}}\left[j_{1}\right](-1)^{j_{1}+j_{2}+j_{3}}\left\{\begin{array}{lll}
j_{1} & j_{2} & j_{3} \\
j_{1}^{\prime} & j_{3} & j_{2}
\end{array}\right\}
$$




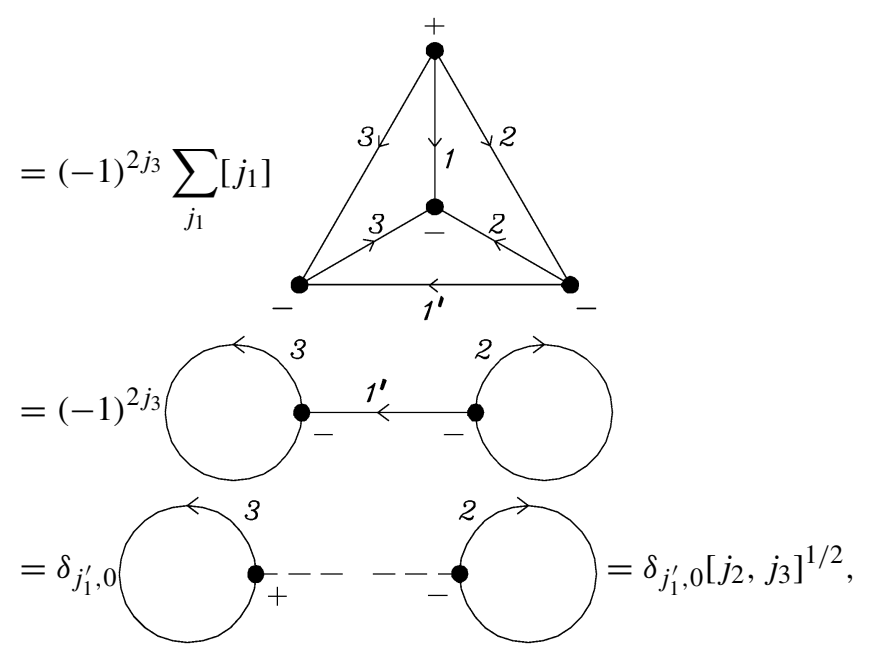

where we have used the separation rule (105) and the zero line removal rule (97).

The well-known orthogonality relation for $6 j$-symbols, which states that, apart from the factor $\left[j_{1}, j_{1}^{\prime}\right]^{1 / 2}$, the $6 j$-symbols form an orthogonal matrix for fixed $j_{2}, j_{3}, j_{2}^{\prime}$, and $j_{3}^{\prime}$,

$$
\sum_{j_{1}}\left[j_{1}\right]\left\{\begin{array}{lll}
j_{1} & j_{2} & j_{3} \\
j_{1}^{\prime} & j_{2}^{\prime} & j_{3}^{\prime}
\end{array}\right\}\left\{\begin{array}{lll}
j_{1} & j_{2} & j_{3} \\
j_{1}^{\prime \prime} & j_{2}^{\prime} & j_{3}^{\prime}
\end{array}\right\}=\delta_{j_{1}^{\prime}, j_{1}^{\prime \prime}}\left[j_{1}^{\prime}\right]^{-1}\left\{j_{1}^{\prime}, j_{2}^{\prime}, j_{3}\right\}\left\{j_{1}^{\prime}, j_{2}, j_{3}^{\prime}\right\},
$$

can also be easily proved. We simply use the rule (109) for a separation over the four lines in reverse direction (note that the required phase change equals unity) obtaining
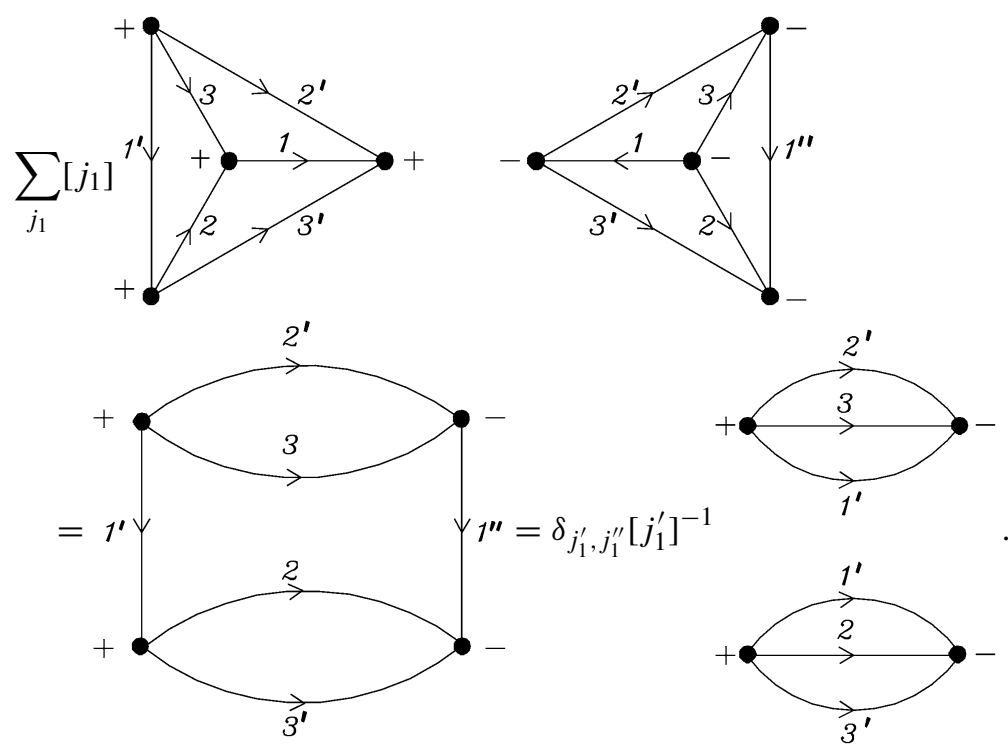

Similarly, the well-known sum rule

$$
\sum_{j_{1}}\left[j_{1}\right](-1)^{j_{1}+j_{1}^{\prime}+j_{1}^{\prime \prime}}\left\{\begin{array}{lll}
j_{1} & j_{2} & j_{3} \\
j_{1}^{\prime} & j_{2}^{\prime} & j_{3}^{\prime}
\end{array}\right\}\left\{\begin{array}{lll}
j_{1} & j_{2} & j_{3} \\
j_{1}^{\prime \prime} & j_{3}^{\prime} & j_{2}^{\prime}
\end{array}\right\}=\left\{\begin{array}{lll}
j_{1}^{\prime \prime} & j_{2} & j_{2}^{\prime} \\
j_{1}^{\prime} & j_{3} & j_{3}^{\prime}
\end{array}\right\}
$$


can also be derived by the use of the following version of the separation rule (109), where now, in view of equation (108), the vertices involving crossing lines have the same signs, so that
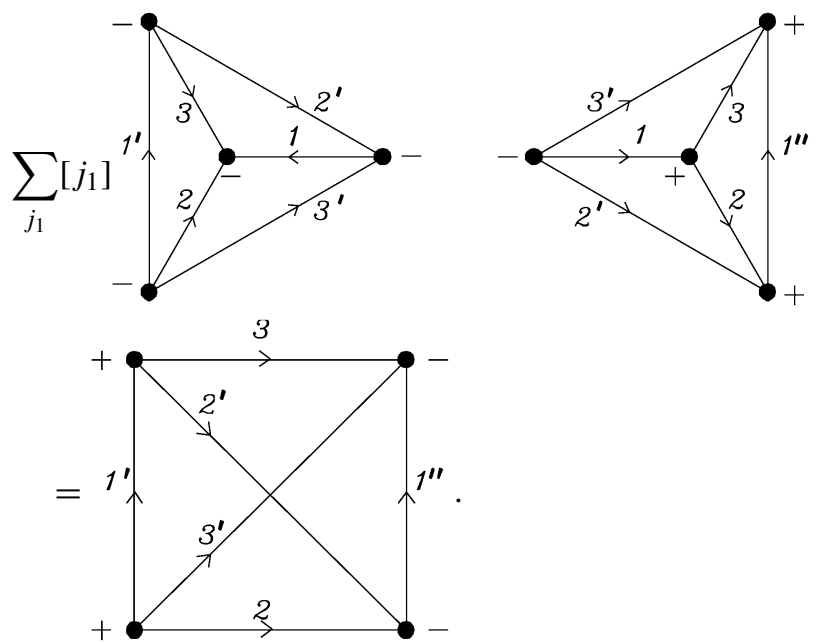

By bringing these $6 j$-diagrams to their canonical form and dividing out the phases thus arising, equation (122) easily follows.

We also see that the $6 j$-diagram reduces to the $3 j$-type oyster diagram when one of the angular momenta vanishes. Thus, applying the rule (96) for the zero line removal, we find that

$$
\left\{\begin{array}{ccc}
j_{1} & j_{2} & j_{3} \\
j_{1}^{\prime} & j_{2}^{\prime} & 0
\end{array}\right\}=\delta_{j_{1} j_{2}^{\prime}} \delta_{j_{2} j_{1}^{\prime}}(-1)^{j_{1}+j_{2}+j_{3}}\left[j_{1}, j_{2}\right]^{-1 / 2}\left\{j_{1}, j_{2}, j_{3}\right\}
$$

\subsection{Properties of the $9 j$-symbol}

The $9 j$-symbol is defined as a contraction of six $3 j m$-symbols, equation (116), and can be computed as a triple sum over the magnetic quantum numbers of the product of these $3 \mathrm{jm}$-symbols. An alternative route is to express it as a single sum over a product of three $6 j$-symbols,

$$
\begin{aligned}
& \left\{\begin{array}{lll}
j_{1} & j_{2} & j_{3} \\
j_{1}^{\prime} & j_{2}^{\prime} & j_{3}^{\prime} \\
j_{1}^{\prime \prime} & j_{2}^{\prime \prime} & j_{3}^{\prime \prime}
\end{array}\right\} \\
& =\sum_{x}[x](-1)^{R+2 x}\left\{\begin{array}{lll}
j_{1} & j_{2} & j_{3} \\
x & j_{1}^{\prime \prime} & j_{1}^{\prime}
\end{array}\right\}\left\{\begin{array}{lll}
j_{1}^{\prime} & j_{2}^{\prime} & j_{3}^{\prime} \\
j_{2}^{\prime \prime} & x & j_{2}
\end{array}\right\}\left\{\begin{array}{ccc}
j_{1}^{\prime \prime} & j_{2}^{\prime \prime} & j_{3}^{\prime \prime} \\
j_{3}^{\prime} & j_{3} & x
\end{array}\right\},
\end{aligned}
$$

where

$$
R=\sum_{i=1}^{3}\left(j_{i}+j_{i}^{\prime}+j_{i}^{\prime \prime}\right) .
$$

This expression can be proved graphically as shown in Fig. 5 .

The $9 j$-symbol has a symmetry group of order 72 , i.e., there are 72 operations permuting its quantum numbers that conserve its absolute value. In the first place, it is invariant under 

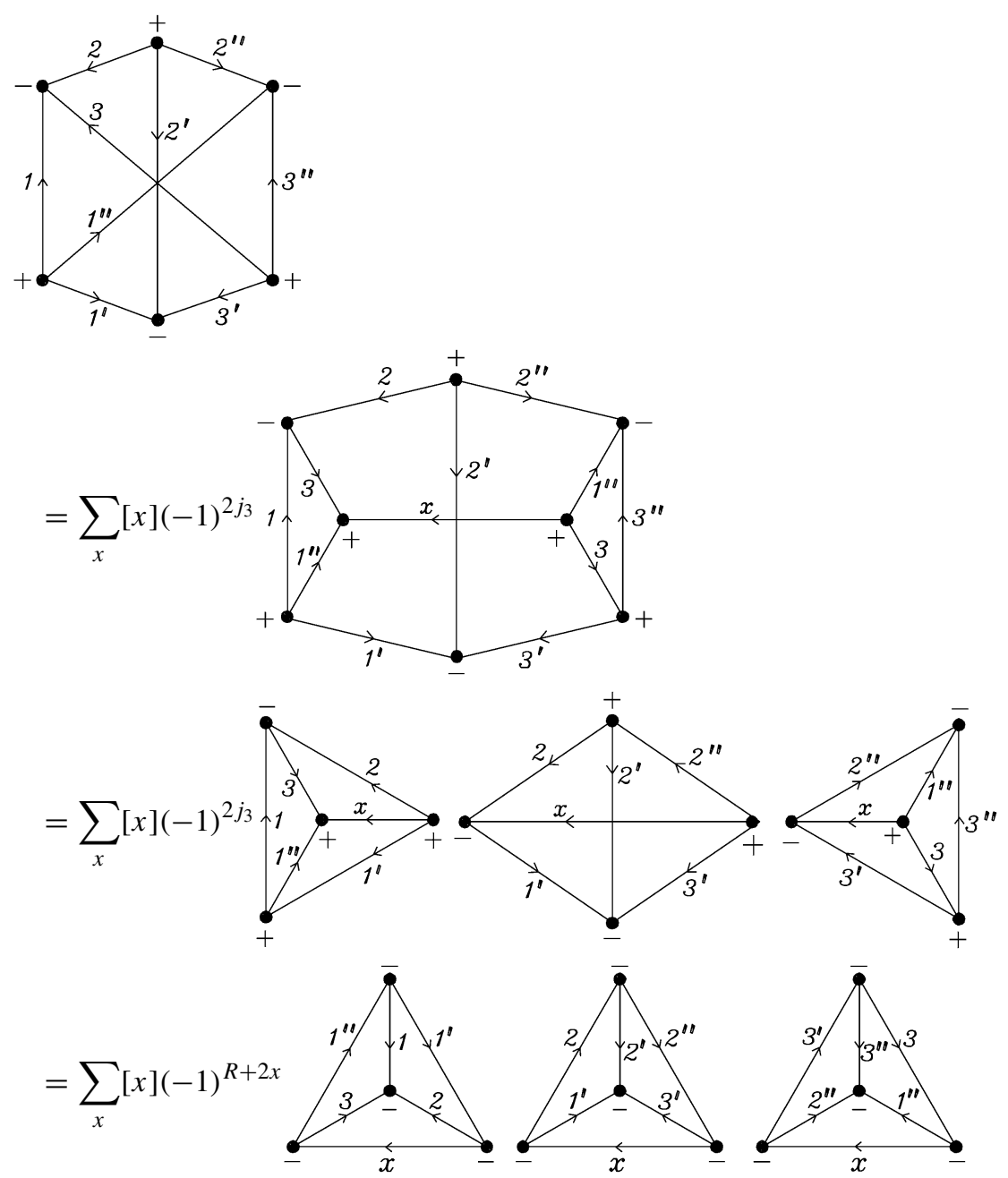

Fig. 5. Graphical derivation of equation (123), which gives a $9 j$-symbol as a sum of a product of three $6 j$-symbols.

cyclic permutations of its rows and columns. This follows immediately from the definition, equation (116), which does not specify the $j$-value that should appear in the upper left-hand corner of the $9 j$-symbol, and from the three-fold symmetry of the corresponding unlabeled $9 j$-diagram. Thus

$$
\left\{\begin{array}{lll}
j_{1} & j_{2} & j_{3} \\
j_{1}^{\prime} & j_{2}^{\prime} & j_{3}^{\prime} \\
j_{1}^{\prime \prime} & j_{2}^{\prime \prime} & j_{3}^{\prime \prime}
\end{array}\right\}=\left\{\begin{array}{lll}
j_{1}^{\prime} & j_{2}^{\prime} & j_{3}^{\prime} \\
j_{1}^{\prime \prime} & j_{2}^{\prime \prime} & j_{3}^{\prime \prime} \\
j_{1} & j_{2} & j_{3}
\end{array}\right\}=\left\{\begin{array}{lll}
j_{2} & j_{3} & j_{1} \\
j_{2}^{\prime} & j_{3}^{\prime} & j_{1}^{\prime} \\
j_{2}^{\prime \prime} & j_{3}^{\prime \prime} & j_{1}^{\prime \prime}
\end{array}\right\}=\cdots
$$

A transposition of the first two columns, however, changes the phase since

$$
\left\{\begin{array}{lll}
j_{1} & j_{2} & j_{3} \\
j_{1}^{\prime} & j_{2}^{\prime} & j_{3}^{\prime} \\
j_{1}^{\prime \prime} & j_{2}^{\prime \prime} & j_{3}^{\prime \prime}
\end{array}\right\}=(-1)^{R}\left\{\begin{array}{lll}
j_{2} & j_{1} & j_{3} \\
j_{2}^{\prime} & j_{1}^{\prime} & j_{3}^{\prime} \\
j_{2}^{\prime \prime} & j_{1}^{\prime \prime} & j_{3}^{\prime \prime}
\end{array}\right\} \text {. }
$$


This is proved graphically as follows:
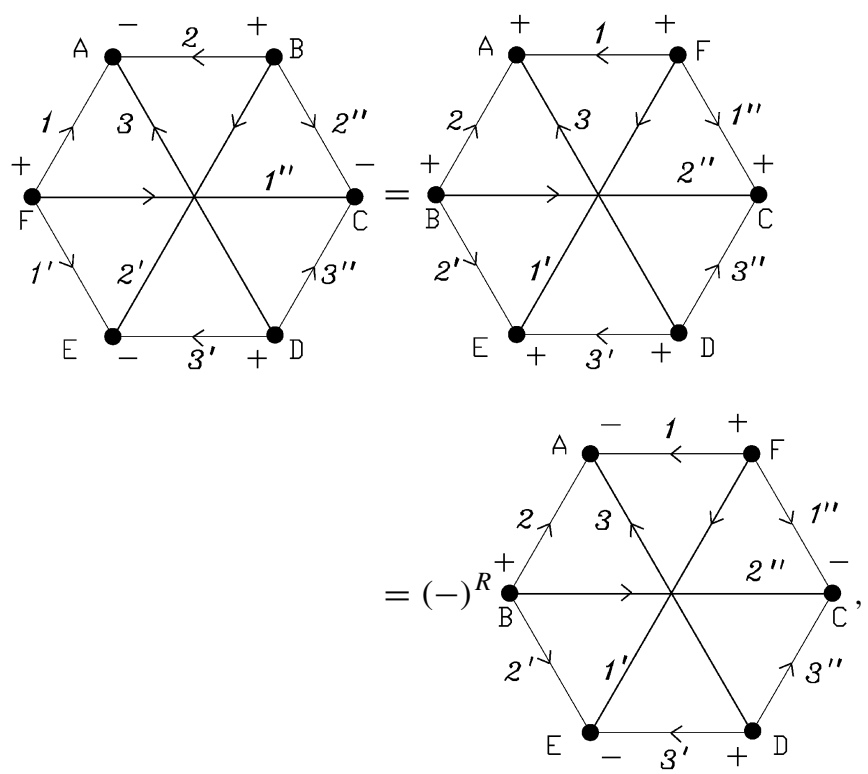

where $R \equiv j_{1}+j_{2}+j_{3}+j_{1}^{\prime}+j_{2}^{\prime}+j_{3}^{\prime}+j_{1}^{\prime \prime}+j_{2}^{\prime \prime}+j_{3}^{\prime \prime}$, equation (124), is due to the sign reversal at nodes A, E, and C. Since a transposition and a cyclic permutation generate the whole permutation group $S_{3}$, this rule holds for all transpositions of the columns.

Any closed diagram is invariant under a simultaneous reversal of all arrows and all node signs. If we perform this operation on the $9 j$-diagram, we interchange the rows and columns of the $9 j$-symbol (i.e., we transpose the corresponding $3 \times 3$ matrix). Hence, the $9 j$-symbol is invariant with respect to a simultaneous interchange of its rows and columns, which implies that it obtains the phase $(-1)^{R}$ under an odd permutation of its rows. Thus, to summarize:

The 9j-symbol is invariant under any even permutation of its rows or columns and obtains the phase $(-1)^{R}$ in case of an odd permutation of its rows or columns.

The graphical technique can be again very useful in deriving various sum rules for the $9 j$-symbols. We leave this to the reader as an exercise.

Finally, if one of the angular momenta vanishes, a $9 j$-symbol reduces to a $6 j$-symbol. For example, when $j_{3}^{\prime \prime}=0$, we get

$$
\left\{\begin{array}{ccc}
j_{1} & j_{2} & j_{3} \\
j_{1}^{\prime} & j_{2}^{\prime} & j_{3}^{\prime} \\
j_{1}^{\prime \prime} & j_{2}^{\prime \prime} & 0
\end{array}\right\}=\delta_{j_{3} j_{3}^{\prime}} \delta_{j_{1}^{\prime \prime} j_{2}^{\prime \prime}}(-1)^{j_{2}+j_{3}+j_{1}^{\prime}+j_{1}^{\prime \prime}}\left[j_{3}, j_{1}^{\prime \prime}\right]^{-1 / 2}\left\{\begin{array}{ccc}
j_{1} & j_{2} & j_{3} \\
j_{2}^{\prime} & j_{1}^{\prime} & j_{1}^{\prime \prime}
\end{array}\right\}
$$


We again easily verify this rule graphically, since

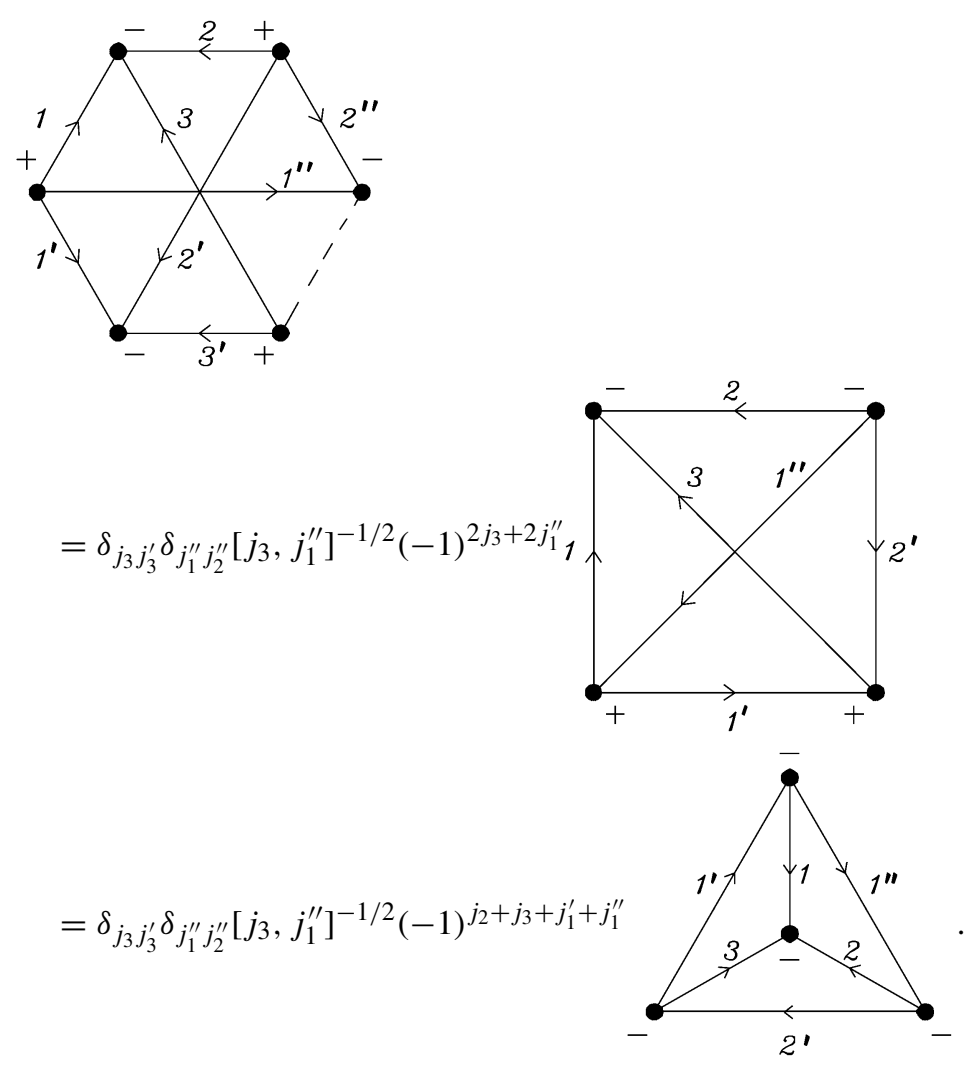

\subsection{Higher $3 n j$-symbols}

The number of closed irreducible diagrams rapidly increases with increasing $n$. For $n \geqslant 4$ there exist two $3 n j$-symbols, whose symmetries can be displayed by writing their arguments on a cylindrical band. In the case of a $3 n j$-symbol of the first kind the band is twisted (a Möbius strip) and in the case of a $3 n j$-symbol of the second kind, it is not. For $n=4$ these are the only irreducible diagrams, but when $n>4$ there exist additional, less symmetric, irreducible $3 n j$-symbols. It can be shown that there are five $15 j$-symbols, $1818 j$-symbols, $8421 j$-symbols, and $57624 j$-symbols, indicating the rapid increase in their number. Many of these symbols, their symmetries, reductions and sum rules have been examined in detail by Jucys and co-workers [18,19]. Should we encounter any one of such diagrams, we shall express them as sums over the products of lower-order $3 n j$-symbols as we did above for the $6 j$ - and $9 j$-symbols.

\section{CONCLUDING REMARKS}

In closing this exposition of the graphical methods of angular momentum theory we wish to briefly point out some of the applications of this technique, particularly as it pertains to the 
field of the molecular electronic structure investigations. Clearly, the angular momentum theory is relevant in any study of rotationally invariant systems and motion in isotropic space, ranging from elementary particle and both classical and quantum field theories to atomic and molecular structure investigations.

Of particular interest and utility are those applications in which this approach is closely linked with other graphical or diagrammatic techniques. Typical representatives of such approaches are those based on Feynman-like or many-body perturbation theory (MBPT) diagrams, which enable an efficient and "graphic" pictorial implementation of the timeindependent Wick theorem in the framework of the second quantization formalism. In the case of atomic systems, both the orbital and spin angular momentum can be handled in this way [31-33], while in molecular studies one is most often concerned with spin couplings, leading to the so-called spin-adapted version of various approaches to the molecular electronic structure.

Perhaps the simplest exploitation of the latter kind deals with the direct evaluation of the spin-adapted configuration interaction (CI) or shell model matrix elements when considering only low-excitation orders (see, e.g., Refs. [31-35]). A more general exploitation of this type is then based on the permutation or symmetric group representation theory [36] or on the unitary group approach (UGA) (see, e.g., Refs. [25,37-39]). The graphical methods also proved to be very useful in elucidating the relationship between the permutation group $\mathrm{S}_{N}$ and unitary group $\mathrm{U}(n)$ approaches [40-42]. In fact, the diagrammatic method is very convenient to compute spin eigenstates adapted to canonical subgroup chains embedded within $\mathrm{S}_{N}$ or $\mathrm{U}(n)$ and Hamiltonian matrix elements between such states [43].

Although in UGA one can completely rely on the algebraic approach [25,37-39], a realization that the Gel'fand-Tsetlin states are equivalent (up to a phase factor) to those arising from the Yamanouchi-Kotani coupling scheme enabled an efficient handling of the two-electron contributions in UGA or graphical UGA (GUGA) [44] via the introduction of suitable "segment values" [45-48]. (Note, however, that GUGA is based on a very different type of diagrams, which not only enable a global representation of the two-column Gel'fand-Tsetlin basis but afford much insight as well.)

Similarly as in variational CI approaches, the diagrammatic technique described in this work has also been successfully exploited when designing the spin-adapted versions of the coupled cluster (CC) formalism, both at the single-reference [49-52] and multireference [53,54] levels, as well as in related approaches [55,56].

In the theory of long-range intermolecular forces angular momentum recoupling plays an important role due to the fact that the multipole expansion of the intermolecular part of the electronic Hamiltonian appears naturally in terms of coupled spherical tensor operators (the multipoles). In second- and higher-order perturbation theory it is convenient to precouple the multipoles on each monomer to irreducible monomer tensors. This requires a recoupling of the multipoles arising in the multipole expansion [57]. This recoupling was applied by Piecuch [58-62] to systems consisting of $N$ closed-shell monomers through third-order of intermolecular perturbation. The graphical technique proved indispensable to handle the very complicated angular momentum algebra arising in this work. Also the theory of long-range interaction-induced properties of collisional complexes [63] benefited greatly from the aid of the diagrammatic technique. Recently the long-range interaction energy between atoms in arbitrary angular momentum states was worked out [64]. Here it was recognized diagrammatically that an $18 j$-symbol appeared, which was a 
useful insight because it showed that the complicated expression was simplified to the utmost.

Finally, the graphical methods of angular momentum coupling proved to be useful even in the analysis of experimental data, as exemplified by the studies of angular distribution in photoionization experiments. For example, they facilitate the derivation of the phenomenological equations for the analysis of angular distribution of photoelectrons from resonant two-photon ionization of alkali atoms with hyperfine interactions by relying on the graphical method and the so-called Liouville representation [65]. As stated in the just quoted paper [65], "graphical representation helps one to visualize the physics and simplifies and organizes the computations".

\section{ACKNOWLEDGEMENT}

One of the authors (J. Paldus) wishes to acknowledge the continued support by the National Science and Engineering Research Council of Canada.

\section{APPENDIX: SUMMARY OF THE GRAPHICAL RULES}

In this section the most important diagrammatic rules are reviewed.

\section{Basic units}

Vectors and arrow directions:

$$
\begin{array}{rlrl}
|j m\rangle & =\longleftrightarrow, & & \mid \vartheta(j m\rangle)=(-1)^{j-m}|j,-m\rangle=\longrightarrow 0, \\
\langle j m|=\gg 0, & & \langle\vartheta(j m)|=(-1)^{j-m}\langle j,-m|=\longleftrightarrow 0 .
\end{array}
$$

Tensor products:

Orthonormality:

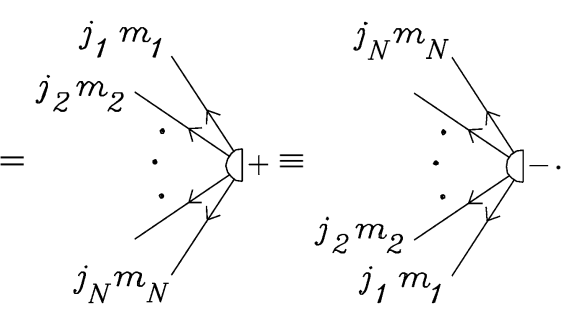

$$
\left\langle j m \mid j^{\prime} m^{\prime}\right\rangle=\delta_{j j^{\prime}} \delta_{m m^{\prime}}=\stackrel{j m}{\longrightarrow} \quad \stackrel{j^{\prime} m^{\prime}}{\longrightarrow} .
$$

\section{Basic rules}

Contractions:

$$
\sum_{m} \mathrm{D} \rightarrow^{j m} \stackrel{j m}{\longrightarrow} \mathrm{O}=\mathrm{D} \stackrel{j}{ }
$$




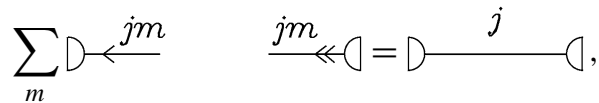

$$
\begin{aligned}
& \sum_{m} \mathrm{D} \rightarrow^{j m} \stackrel{j m}{\rightarrow} \mathrm{O}=\mathrm{D} \stackrel{j}{\rightarrow} \mathrm{O}, \\
& \sum_{m} \mathrm{D} \gg^{j m} \quad \stackrel{j m}{\gg} \mathrm{O}=\mathrm{D} \stackrel{j}{\longleftarrow} \mathrm{O} \text {. }
\end{aligned}
$$

Arrow reversals:

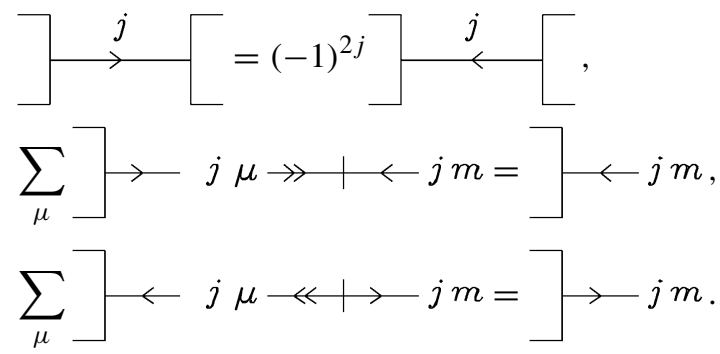

\section{$1 j m$-symbol}

$$
\begin{aligned}
& \left(\begin{array}{c}
j \\
m^{\prime} m
\end{array}\right) \equiv\left\langle j m^{\prime} \mid \vartheta(j m)\right\rangle \\
& \left(\begin{array}{c}
j \\
m m^{\prime}
\end{array}\right) \equiv\left\langle\vartheta\left(j m^{\prime}\right) \mid j m\right\rangle=(-1)^{2 j}\left\langle j m^{\prime} \mid \vartheta(j m)\right\rangle=(-1)^{2 j}\left(\begin{array}{c}
j \\
m^{\prime} m
\end{array}\right), \\
& \stackrel{j m^{\prime}}{\longleftrightarrow},{ }^{j m}=(-1)^{2 j} \stackrel{j m^{\prime}}{\longrightarrow}, \stackrel{j m}{\longleftarrow}, \\
& \left\langle j m^{\prime} \mid \vartheta(j m)\right\rangle=\stackrel{j m^{\prime}}{\longrightarrow} \mid \stackrel{j m}{\longleftarrow}=(-1)^{j+m^{\prime}} \delta_{m^{\prime},-m}=(-1)^{j-m} \delta_{m^{\prime},-m}, \\
& \left\langle\vartheta\left(j m^{\prime}\right) \mid j m\right\rangle=\frac{j m^{\prime}}{\longleftrightarrow \quad+\quad j^{j m}}=(-1)^{j-m^{\prime}} \delta_{m^{\prime},-m}=(-1)^{j+m} \delta_{m^{\prime},-m} .
\end{aligned}
$$

\section{Clebsch-Gordan coefficients and diagrams}

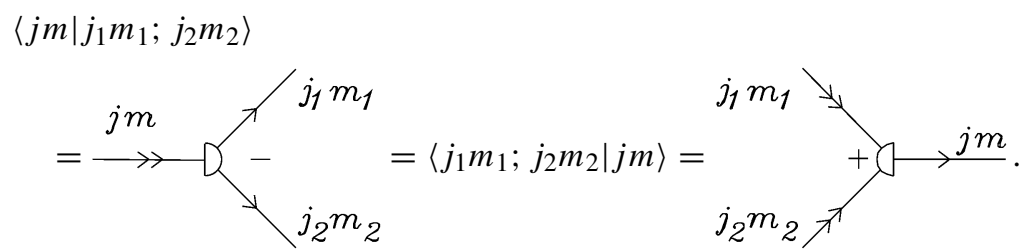




\section{Symmetries:}

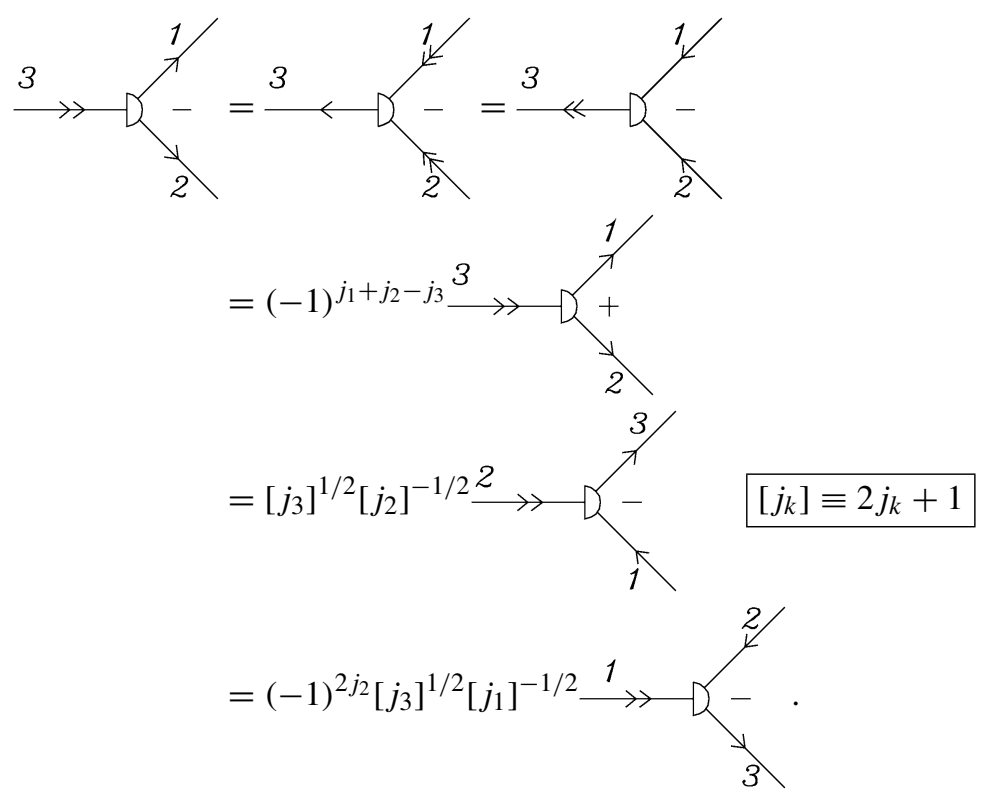

$3 j m$-symbols and diagrams

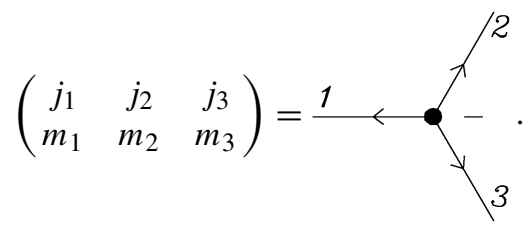

Symmetries:

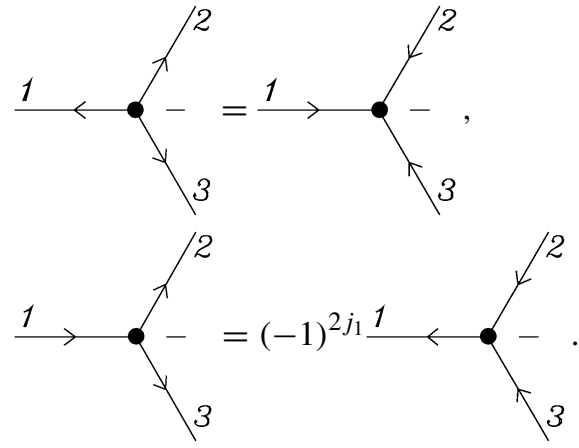

(The line with unique variance determines the phase obtained by reversal of all arrows.)

To raise a magnetic quantum number contract the $3 j m$-symbol with the $1 j m$-symbol on the right; to lower a magnetic quantum number contract the $3 j \mathrm{~m}$-symbol with the $1 \mathrm{jm}$-symbol on the left. 
EXAMPLE.

$$
\begin{gathered}
\sum_{m}\left(\begin{array}{ccc}
j_{1} & j_{2} & j_{3} \\
m & m_{2} & m_{3}
\end{array}\right)\left(\begin{array}{c}
j_{1} \\
m m_{1}
\end{array}\right)=\left(\begin{array}{ccc}
m_{1} & j_{2} & j_{3} \\
j_{1} & m_{2} & m_{3}
\end{array}\right) \\
\sum_{m}+j_{3}+j_{3}+j_{1}=+m_{1} m_{1}
\end{gathered}
$$

Raising and lowering can be performed on any column independently.

Vertex sign reversal:

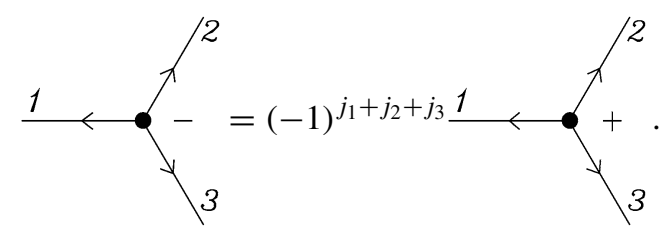

\section{Transition from CG to $3 j m$-diagrams}

Apply the following operations in order to convert a CG into a $3 \mathrm{jm}$-vertex:

1. Conserve variances (arrow directions).

2. Invert vertex sign.

3. Multiply by $\sqrt{[j]}$, where $j$ labels the unique line of the CG diagram.

4. Multiply for every outgoing double arrow by $(-1)^{2 j}$, where $j$ is the label of the doublearrowed line.

5. Multiply also by $(-1)^{2 j}$, where $j$ is the first non-unique line met when going around the vertex starting at the unique line. If the unique line is a ket we go in the direction of the node sign. If the unique line is a bra ('complex') we go contra the direction of the node sign.

See Table 1 in Section 3.6 for illustrations of CG to $3 j m$-transitions.

\section{Diagram manipulation}

Closed boxes do not have free lines other than the ones shown explicitly, open boxes can have any number of free lines.

Removal of a zero line:

Let lines go in the direction indicated by the vertex sign, then take the vertex out:

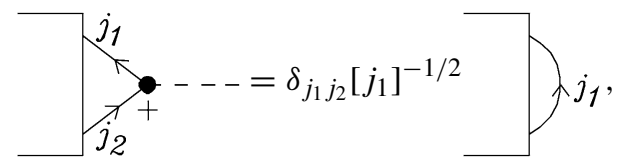




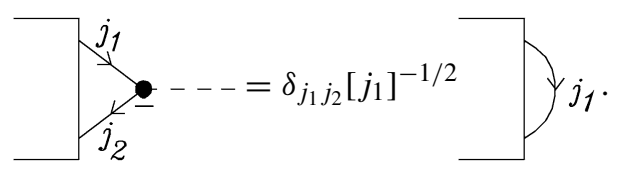

Exception to this rule:

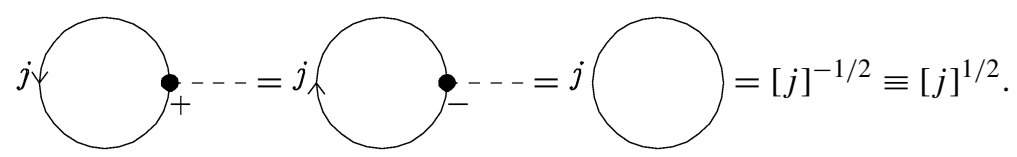

Insertion of a summation line:

Let $j_{1}$ and $j_{2}$ run in the same direction; $x$ then runs in the opposite direction. Choose vertex signs such that the three lines have the same order on both new vertices. This rule holds for crossing lines as well

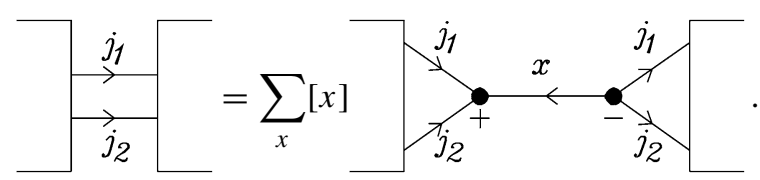

Separation over one line:

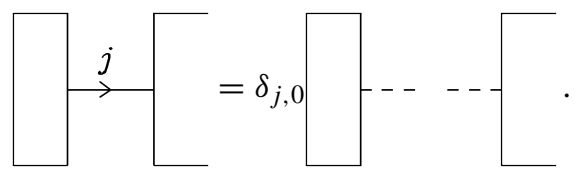

Separation over two lines:

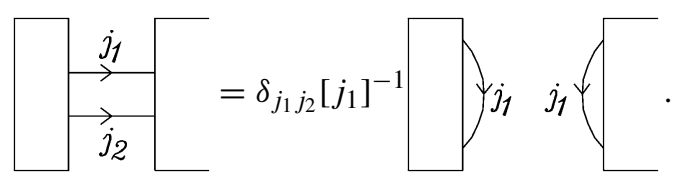

Separation over three lines:

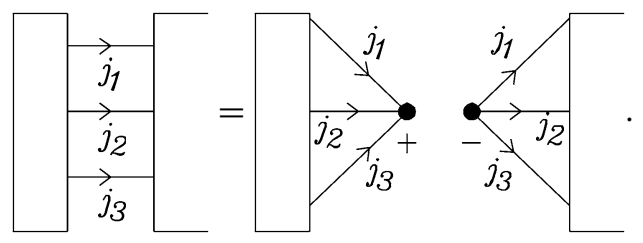

In rules (125)-(127) the diagrams on the right-hand side may be open, and in particular they may be empty. In that case we recover the factorization rules (99), (95), and (101), respectively. 


\section{Basic irreducible diagrams; $3 \boldsymbol{n} \boldsymbol{j}$-symbols}

$n=1 ; 3 j$-symbol:

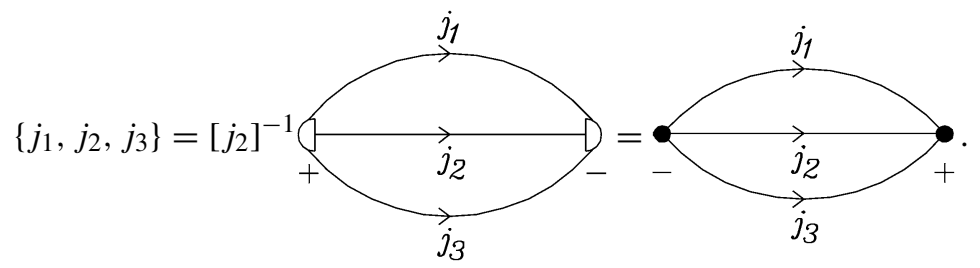

The diagram is totally symmetric under permutations.

$n=2 ; 6 j$-symbol:

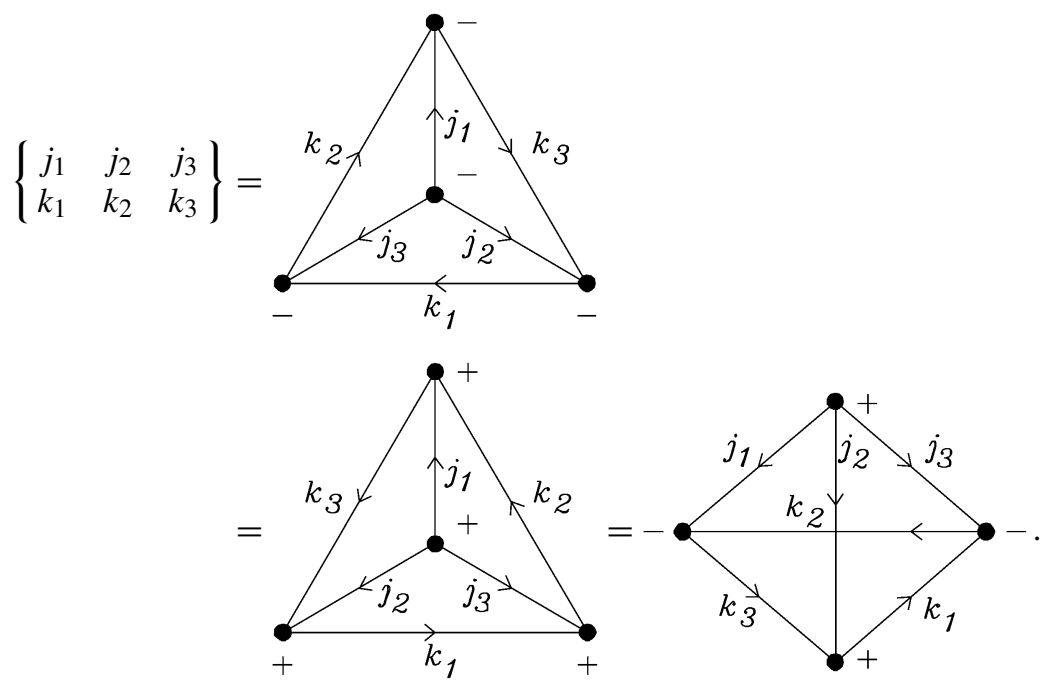

In the "tetrahedral" form of the $6 j$-symbol all node signs are the same, and arrows on the perimeter are directed as indicated by the node signs. Arrows on the central node are either all outgoing or all ingoing. The "diamond" form is obtained from the "tetrahedral" form by pulling the interior vertex outside.

Symmetries:

$$
\begin{aligned}
\left\{\begin{array}{lll}
j_{1} & j_{2} & j_{3} \\
k_{1} & k_{2} & k_{3}
\end{array}\right\} & =\left\{\begin{array}{lll}
j_{a} & j_{b} & j_{c} \\
k_{a} & k_{b} & k_{c}
\end{array}\right\}=\left\{\begin{array}{lll}
j_{a} & k_{b} & k_{c} \\
k_{a} & j_{b} & j_{c}
\end{array}\right\} \\
& =\left\{\begin{array}{lll}
k_{a} & j_{b} & k_{c} \\
j_{a} & k_{b} & j_{c}
\end{array}\right\}=\left\{\begin{array}{lll}
k_{a} & k_{b} & j_{c} \\
j_{a} & j_{b} & k_{c}
\end{array}\right\},
\end{aligned}
$$

where $(a b c)$ is any permutation of $\left(\begin{array}{lll}1 & 2 & 3\end{array}\right)$. 
$n=3 ; 9 j$-symbol:

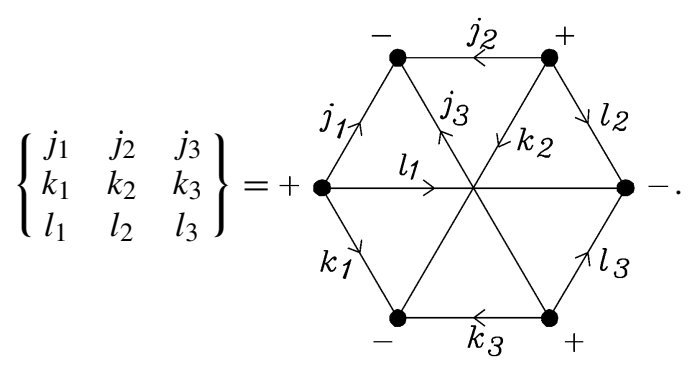

The node signs on the perimeter alternate. The negative nodes give the rows and the positive nodes the columns of the $9 j$-symbol.

Symmetries:

$$
\begin{aligned}
\left\{\begin{array}{lll}
j_{1} & j_{2} & j_{3} \\
k_{1} & k_{2} & k_{3} \\
l_{1} & l_{2} & l_{3}
\end{array}\right\} & =\left\{\begin{array}{lll}
j_{1} & k_{1} & l_{1} \\
j_{2} & k_{2} & l_{2} \\
j_{3} & k_{3} & l_{3}
\end{array}\right\} \\
& =(-1)^{R}\left\{\begin{array}{lll}
j_{a} & j_{b} & j_{c} \\
k_{a} & k_{b} & k_{c} \\
l_{a} & l_{b} & l_{c}
\end{array}\right\}=(-1)^{R}\left\{\begin{array}{lll}
j_{a} & k_{a} & l_{a} \\
j_{b} & k_{b} & l_{b} \\
j_{c} & k_{c} & l_{c}
\end{array}\right\},
\end{aligned}
$$

where

$$
R= \begin{cases}j_{1}+j_{2}+j_{3}+k_{1}+k_{2}+k_{3}+l_{1}+l_{2}+l_{3} & \text { if }(a b c) \text { is odd } \\ 0 & \text { if }(a b c) \text { is even. }\end{cases}
$$

\section{REFERENCES}

[1] R.F.A. Clebsch, Theorie der binären algebraischen Formen, Teubner, Leipzig, 1872.

[2] P. Gordan, Über das Formensystem binären Formen, Teubner, Leipzig, 1875.

[3] J.J. Sylvester, Am. J. Math. 1 (1878) 64.

[4] W.K. Clifford, Am. J. Math. 1 (1878) 126.

[5] P. Gordan, W.G. Alexejeff, Z. Phys. Chem. 35 (1900) 610.

[6] G. Rumer, E. Teller, H. Weyl, Gött. Nachr. Math.-Phys. Klasse 499 (1932).

[7] M.E. Rose, Theory of Angular Momentum, Wiley, New York, 1957.

[8] E.P. Wigner, Group Theory and Its Applications to the Quantum Mechanics of Atomic Spectra, Academic Press, New York, 1959, originally published as Gruppentheorie und ihre Anwendung auf die Quantenmechanik der Atomspektren, Vieweg, Braunschweig, 1931, translated to English by J.J. Griffin.

[9] U. Fano, G. Racah, Irreducible Tensorial Sets, Academic Press, New York, 1959.

[10] A.R. Edmonds, Angular Momentum in Quantum Mechanics, second ed., Princeton Univ. Press, Princeton, 1960.

[11] B.R. Judd, Operator Techniques in Atomic Spectroscopy, McGraw-Hill, New York, 1963.

[12] L.C. Biedenharn, H. van Dam, Quantum Theory of Angular Momentum, Academic Press, New York, 1965.

[13] L.C. Biedenharn, J.D. Louck, Angular Momentum in Quantum Physics, Encyclopedia of Mathematics, vol. 8, Addison-Wesley, Reading, 1981.

[14] D.A. Varshalovich, A.N. Moskalev, V.K. Khersonskii, Quantum Theory of Angular Momentum, World Scientific, Singapore, 1988, originally published in Russian by Nauka, Leningrad, 1975.

[15] R.N. Zare, Angular Momentum: Understanding Spatial Aspects in Chemistry and Physics, Wiley, New York, 1988.

[16] M. Danos, V. Gillet, Angular Momentum Calculus in Quantum Physics, World Scientific, Singapore, 1990. 
[17] J.D. Louck, in: G.W.F. Drake (Ed.), Atomic, Molecular, \& Optical Physics Handbook, Am. Inst. of Phys, Woodbury, NY, 1996, pp. 6-55.

[18] A. Jucys, I.B. Levinson, V.V. Vanagas, The Theory of Angular Momentum, Israel Program for Scientific Translation, Jerusalem, 1962, translated from the 1960 Russian edition by A. Sen and A.R. Sen.

[19] A. Jucys, A.A. Bandzaitis, Theory of Angular Momentum in Quantum Mechanics, Lithuanian Academy of Sciences, Vilnius, 1965 [in Russian].

[20] A. El Baz, B. Castel, Graphical Methods of Spin Algebras, Dekker, New York, 1972.

[21] D.M. Brink, G.R. Satchler, Angular Momentum, third ed., Clarendon, Oxford, 1993.

[22] E.P. Wigner, reprinted in: Biedenharn and van Dam [12].

[23] W. Pauli, Z. Phys. 43 (1927) 601.

[24] J.S. Schwinger, reprinted in: Biedenharn and van Dam [12].

[25] J. Paldus, in: H. Eyring, D. Henderson (Eds.), Theoretical Chemistry: Advances and Perspectives, vol. 2, Academic Press, New York, 1976, pp. 131-290.

[26] P. Gordan, J. Reine Angew. Math. 69 (1869) 1.

[27] B.L. van der Waerden, Die gruppentheoretische Methode in der Quantenmechanik, Springer-Verlag, Berlin, 1932.

[28] I. Lindgren, J. Morrison, Atomic Many-Body Theory, second ed., Springer-Verlag, New York, 1986.

[29] G. Racah, Phys. Rev. 62 (1942) 438.

[30] T. Regge, Nuovo Cimento 11 (1959) 116.

[31] V.V. Tolmachev, Adv. Chem. Phys. 14 (1969) 471

[32] J.S. Briggs, Rev. Mod. Phys. 43 (1971) 189.

[33] B.G. Adams, J. Paldus, Phys. Rev. A 24 (1981) 2302.

[34] J. Paldus, B.G. Adams, J. Č́žžek, Int. J. Quantum Chem. 11 (1977) 813.

[35] B.G. Adams, J. Paldus, J. Čížek, Int. J. Quantum Chem. 11 (1977) 849.

[36] J. Paldus, P.E.S. Wormer, Phys. Rev. A 18 (1978) 827.

[37] J. Paldus, J. Chem. Phys. 61 (1974) 5321.

[38] J. Paldus, in: D.G. Truhlar (Ed.), Mathematical Frontiers in Computational Chemical Physics, IMA Series, vol. 15, Springer-Verlag, Berlin, 1988, pp. 262-299.

[39] J. Paldus, Contemp. Math. 160 (1994) 209.

[40] P.E.S. Wormer, J. Paldus, Int. J. Quantum Chem. 16 (1979) 1307.

[41] J. Paldus, P.E.S. Wormer, Int. J. Quantum Chem. 16 (1979) 1321.

[42] P.E.S. Wormer, J. Paldus, Int. J. Quantum Chem. 18 (1980) 841.

[43] P.E.S. Wormer, in: M.F. Guest, S. Wilson (Eds.), Proceedings of the Daresbury Study Weekend, Science Research Council, Daresbury, Warrington, 1979, pp. 49-59.

[44] I. Shavitt, in: D.G. Truhlar (Ed.), Mathematical Frontiers in Computational Chemical Physics, IMA Series, vol. 15, Springer-Verlag, Berlin, 1988, pp. 300-349.

[45] J. Paldus, M.J. Boyle, Physica Scripta 21 (1980) 295.

[46] J. Paldus, in: M.F. Guest, S. Wilson (Eds.), Proceedings of the Daresbury Study Weekend, Science Research Council, Daresbury, Warrington, 1980, pp. 40-48.

[47] J. Paldus, M.J. Boyle, Phys. Rev. A 22 (1980) 2299.

[48] M.J. Boyle, J. Paldus, Phys. Rev. A 22 (1980) 2316.

[49] J. Paldus, J. Chem. Phys. 67 (1977) 303.

[50] B.G. Adams, J. Paldus, Phys. Rev. A 20 (1979) 1.

[51] M. Takahashi, J. Paldus, J. Chem. Phys. 85 (1986) 1486.

[52] P. Piecuch, J. Paldus, Int. J. Quantum Chem. 36 (1989) 429.

[53] P. Piecuch, J. Paldus, Theor. Chim. Acta 83 (1992) 69.

[54] P. Piecuch, J. Paldus, J. Chem. Phys. 101 (1994) 5875

[55] P. Piecuch, J. Paldus, Theor. Chim. Acta 78 (1990) 65.

[56] A.E. Kondo, P. Piecuch, J. Paldus, J. Chem. Phys. 102 (1995) 6511.

[57] P.E.S. Wormer, Ph.D. thesis, University of Nijmegen, 1975.

[58] P. Piecuch, Int. J. Quantum Chem. 25 (1984) 449.

[59] P. Piecuch, J. Math. Phys. 27 (1986) 2165.

[60] P. Piecuch, in: J. Maruani (Ed.), Molecules in Physics, Chemistry and Biology, Topics in Molecular Organization and Engineering, vol. 2, Kluwer Academic, Dordrecht, 1988, pp. 417-505.

[61] P. Piecuch, Mol. Phys. 66 (1989) 805.

[62] P. Piecuch, Acta Phys. Pol. A 77 (1990) 453.

[63] T.G.A. Heijmen, R. Moszynski, P.E.S. Wormer, A. van der Avoird, Mol. Phys. 89 (1996) 81.

[64] R.V. Krems, G.C. Groenenboom, A. Dalgarno, J. Phys. Chem. A 108 (2004) 8941.

[65] R.-L. Chien, J. Chem. Phys. 81 (1984) 4023. 


\section{Note added in proof}

The diagrammatic technique of angular momentum theory can be, in principle, generalized to handle Racah-Wigner algebras of arbitrary compact groups. In this regard, we refer an interested reader to the monograph by Stedman [1] and to an extensive paper by Kibler and El Baz [2] and references therein. However, in this work we restrict our attention to the group $\mathrm{SU}(2)$ and focus on the exploitation of graphical techniques of spin algebras for the angular momentum problems arising in atomic and molecular physics.

\section{References}

[1] G. E. Stedman, Diagram Techniques in Group Theory (Cambridge University Press, Cambridge, 1990).

[2] M. Kibler and E. El Baz, Int. J. Quantum Chem. 16, 1161 (1979).

\section{Erratum}

Entry 4 of Table 1 on page 31 should read:

4.

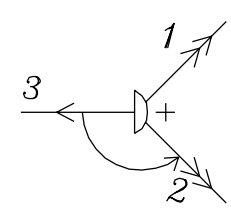

